\title{
Neutrino Interactions in a Hybrid \\ Emulsion - Bubble Chamber Detector
}

by

Robert Ludwig Rosenbladt

A dissertation submitted in partial fulfillment

of the requirements for the degree of

Doctor of Philosophy

University of Washington

1981

Approved by

(Chairperson of Supervisory Committee)

Program Authorized to Offer Degree

Physics

Date 


\section{Doctoral Dissertation}

In presenting this dissertation in partial fulfillment of the requirements for the Doctoral degree at the University of Washington, I agree that the Library shall make its copies freely available for inspection. I further agree that extensive copying of this dissertation is allowable only for scholarly purposes. Requests for copying or reproduction of the dissertation may be referred to University Microfilms, 300 North Zeeb Road, Ann Arbor, Michigan 48106 , or to the author.

Signature

Date 


\title{
University of Washington
}

Abstract

\author{
NEUTRINO INTERACTIONS IN A HYBRID \\ EMULSION - BUBBLE CHAMBER DETECTOR
}

by Robert Ludwig Rosenbladt

Chairperson of the Supervisory Comittee:

Professor Jere J. Lord

Department of Physics

A target consisting of 22 - 1 11ter stacks of cryogenically sensitive nuclear emulsion has been exposed 1nside the 15 Foot Bubble Chamber to the Fermilab wide-band neutrino beam. A hybrid system of emulsion plus bubble chamber was used to find and analyze neutrino interactions with nuclei in the emulsion target.

The average multiplicity of charged minimum ionization tracks of the 45 events was found to be $6.8 \pm 0.5$. The normalized multiplicity with respect to neutrino - proton interactions at the same average hadronic center of mass energy was found to be $1.3 \pm 0.2$. When compared to neutrino - proton interactions, the rapidity distribution shows a clear signal for intranuclear cascading in the target fragmentation region. Measured rapidity and multiplicity distributions are compared with predictions of the Growth of Longitudinal Distances Model of Nikolaev and the Coherent Tube Model.

In one event has been observed the decay of a charged particle after a flight path of 50 microns. The decay has been identified as that of $\mathrm{F}^{+}+\pi^{+} \pi^{+} \pi^{-} \pi^{0}$. The decay occurred after a time of $1.4 \times 10^{-13}$ 
second. The mass of the $\mathrm{F}^{+}$has been determined to be $2017 \pm 25 \mathrm{MeV} / \mathrm{c}^{2}$. The decay time and pionic final state are consistent with gluon interactions being significant in the $\mathrm{F}^{+}$decay process. 
TABLE OF CONTENTS

Page

Chapter I: Introduction ................ 1

Chapter II: Experimental Procedures .. . . . . . . 6

Exposure at Fermilab ............... 6

Emulsion Target. . . . . . . . . . ... 8

Mechanical Support . . . . . . . . . . 14

Bubble Chamber Film - Scanning and Copying . . . . 22

Bubble Chamber Predictions ........... 24

Emulsion Scanning. . . . . . . . . . . 26

Chapter III: Multiparticle Production . . . . . . . . 38

Theory and Background. . . . . . . . . . 38

Modified Cascade Model . . . . . . . . . 39

Coherent Tube Mode1. . . . . . . . . . . 40

Growth of Longitudinal Distances Model . . . . . . 40

Events Found .................. 42

Muon Selection and Charged Current Cut ........ 42

Energy Correction. . . . . . . . . . . . 44

Scanning Bias Test .............. 47

Multiplicity Distributions ............ 49

Transverse Momentum Distributions. . . . . . . 58

Pseudo - Rapidity Distributions. . . . . . . . 58

Chapter IV: Production and Decay of $\mathrm{F}^{+}$Meson. . . . . . 73

Theory and Background. . . . . . . . . . 73

Experimental Results ............ 79

Chapter V: Conclusions. . . . . . . . . . . . 92

List of References . . . . . . . . . . . . . 95 
LIST OF FIGURES

Number

Page

2-1. Observed neutrino energy spectrum in the 15-Foot Bubble Chamber [II-1]........... 7

2-2. Location of emulsion boxes with respect to the 15-Foot Bubble Chamber and the EMI . . . . . . . 9

2-3. Orientation of an emulston stack and its scribe lines . . . . . . . . . . . . . . . 11

2-4. Fading of tracks in emulsion stored at $+20^{\circ} \mathrm{C},+5^{\circ} \mathrm{C}$ and $-10^{\circ} \mathrm{C}$............ 13

2-5. Fractional change in length of emulsion pellicle as a function of temperature. . . . . . 15

2-6. Difference between predicted and found track angles. 2-6a is azimuth. 2-6b is dip....... 29

2-7. Difference between predicted and found event locations. 2-7a is the beam direction. $2-7 b$ is the vertical direction. $2-7 c$ is the pellicle number. . . . . . . . . . . 30

2-8. Difference between predicted and found event locations normalized to the quoted error in the prediction. $2-8 a$ is the beam direction. $2-8 b$ is the vertical direction. $2-8 c$ is the pellicle number. . . . . . . . . . . . 31

2-9. Distribution of the event locations in the beam direction - 1.e. depth in emulsion. . . . . . 34

2-10. Distribution of the number of heavily ionizing tracks. Results from this experiment are crosshatched. Results from reference [II-9] are also shown .............. 35

3-1. Distribution of minimum lonizing tracks. . . . . . 43

3-2. Integral distribution of the quantity $\mathrm{P}_{\mathrm{TR}} \cdot$. . . . 45

3-3. Visible and corrected energy probability distributions. The vistble distribution is crosshatched................... 46 
3-4. Kinematic distributions. 3-4a is the center of mass hadronic energy, $W$. $3-4 \mathrm{~b}$ is the square of the four-momentum transferred, $Q^{2}$................. 48

3-5. Average number of shower tracks, $\left(n_{s}\right)$, versus number of heavily ionizing tracks, $\mathrm{N}_{\mathrm{h}}$. Also shown is a least-squares fit to the data. ... 50

3-6. Average center of mass hadronic energy, (W), versus number of heavily lonizing tracks, $N_{h} \ldots . . .51$

3-7. Average number of hadrons, $\left(n_{c}\right)$, versus natural logarithm of $\mathrm{W}^{2}$............. 52

3-8. Normalized multiplicity $R_{A}$, versus atomic weight of the target, $A$ i/ ${ }^{\prime}$ 'Also shown are neon results from reference [III-2]. Predictions from the MCM, CTM and GLD models are also displayed ................ 54

3-9. Dispersion versus the average number of hadrons, $\left(n_{c}\right)$, and linear least squares fit ..... 55

3-10. Distribution of transverse momentum (squared) with respect to the visible hadronic system. . . . . 59

3-11. Pseudo-rapidity distributions of hadrons from charged current events. . . . . . . . . 61 61

3-12. Rapidity distributions from neutrino interactions with emulsion, neon [III-2] and protons [III-21] .. 64

3-13. Comparison of emulsion to proton rapidity distributions in neutrino interactions. a versus rapidity (where $R_{y}=A^{\alpha}$ ). . . . . . . 65

3-14. Pseudo-rapidity distributions of hadrons from this experiment and from muon-emulsion Interactions [III-3].............67

3-15. Pseudo-rapidity distributions of hadrons from this experiment and. from $\pi$-proton interactions at the same CTM effective energy shifted for A dependence within CTM. ........68

3-16. Ratio of pseudo-rapidity distributions of large and small $\mathrm{N}$ data samples versus pseudo-rapidity. $\mathrm{g}$ Also shown is GLD 
Number

prediction for freon at $v=10 \mathrm{GeV}$......... 69

4-1. Feynman diagrams of: 4-1a muon decay, 4-1b semi-1eptonic decay of $D$ or $F$ in spectator model, 4-1c W-exchange in $D^{\circ}$ decay, annihilation in $F$ decay. . . . . . . . 76

4-2. Phase space limits for charm production

in $\mathrm{F}^{+}$event and location of the event

in phase space. . . . . . . . . . . . . 86

4-3. Likelihood function for $\mathrm{F}^{+}$I1fetime calculated from results of this experiment and reference $[I V-10] . . . . . . . . . .89$ 


\section{LIST OF TABLES}

Number

Page

II-1: Chi-square tests of Gaussian and flat

distributions of (predicted-found)

locations of events ..................... 32

III-1: Fits to dispersion versus average

hadron multiplicity ............. 56

III-2: Comparison of number of tracks in bins of

rapidity and pseudo-rapidity. . . . . . . . . 62

IV-1: Measured track parameters for event . . . . . . .81

IV-2: 3-C fits obtained . . . . . . . . . . . 84

IV-3: Fit results for $\mathrm{F}^{+}(2030)+\pi^{+} \pi^{+} \pi^{-} \pi^{0}$, with $\pi^{0}+\gamma_{1} \gamma_{2} \cdot .85$

IV-4: Summary of $\mathrm{F}^{+}$decays seen in emulsion ....... 88 


\section{LIST OF PLATES}

Number

Page

I Emulsion boxes during loading of target . . . . . . . . . 16

II Emulsion boxes installed in the 15-Foot Bubble Chamber. . . . . . . . . . . . . . 18

II Installation of an emulsion stack . . . . . . . . . . . 21

IV Neutrino event with $\mathrm{F}^{+}$decay as seen in emulsion and bubble chamber. . . . . . . . . . 80 


\section{ACKNOWLEDGEMENTS}

I would like to express my sincere appreciation to my advisor, Professor .Jere J. Lord, for his support and guidance during the course of this work. His continuous encouragement has been instrumental in making my graduate career a rewarding experience.

Dr. Louis Voyvodic, the spokesman of this experiment, has been very helpful in establishing an atmosphere at Fermilab which has been conducive towards the education of the junior member of a large collaboration.

I wish to thank $\mathrm{Dr}$. Jeffery Wilkes for the many useful suggestions he has made to me and for his patient guidance. I also wish to express my appreciation to Dr. Steve Anderson and Dr. Terry Koss for numerous fruitful discussions which have been a great deal of assistance to me.

Professor Stephen Ellis and Professor Thompson Burnett have carefully read this dissertation and I am grateful for their many valuable suggestions.

Donna Kubik and Diana Klug have fulfilled an invaluable role in the acquisition of this data. Their enthusiasm during the long hours of scanning and measuring work has been remarkable.

All of my collaborators in E564 have been very helpful and I owe them a great deal of thanks for their cooperation.

The technical assistance of the University of Washington Physics Machine Shop and of the Fermilab staff has been crucial to this work. Lastly, I wish to express my deepest appreclation to my wife, 
Dorothy, for her continuous support and patience during the years of my graduate studies.

This work has been supported In part by the U. S. Department of Energy. 


\section{CHAPTER I}

\section{INTRODUCTION}

This dissertation is based upon neutrino - nucleus interactions from an experiment, E564, performed at the Fermi National Accelerator Laboratory (FNAL) in Batavia, Illinols. A hybrid detector consisting of the 15 Foot Bubble Chamber plus 22 - 1 1iter stacks of emulsion was used for this experiment. The emulsion stacks were located inside the bubble chamber and served as a track sensitive target. This detection system was exposed to the wide band neutrino beam at FNAL between November, 1978 and January, 1979.

The E564 collaboration was comprised of physicists from FNAL, the University of Kansas, and the University of Washington in the U.S.; the Institute of Nuclear Physics - Krakow, Poland; the Institute of Nuclear Research and Nuclear Energy - Sofia, Bulgaria; the Institute of High Energy Physics (IHEP) - Serpukhov, the Institute of Theoretical and Experimental Physics (ITEP) - Moscow, and the Joint Institute of Nuclear Research (JINR) - Dubna in the U.S.S.R. (I-I). Several groups within the collaboration, i.e. FNAL, Krakow and Washington, had previously gained valuable experience in the use of a hybrid emulsion detector from an earlier FNAL muon experiment, E382.

The geometric arrangement of the experiment allows unsurpassed acceptance of the production products of the events. Only $1 / 4^{\prime \prime}$ of stainless steel separates each emulsion stack from the 11quid deuterlum of the bubble chamber. The bubble chamber and its superconducting magnet yield momentum analysis of particles produced in the neutrino 
events, as well as some information on secondary interactions and photon conversions. Measurements of the bubble chamber film also lead to predictions for the location of each tagged neutrino - emulsion interaction. The emulsion, on the other hand, gives a $4 \pi$ steradian view of the interaction vertex with a micron scale of resolution. The low rate for neutrino collisions would present an insurmountable scanning problem for emulsion exposed alone. However, use of emulsion in tandem with the bubble chamber significantly reduces the required scanning volume to a tolerable level. The advantages of this system more than compensate for the disadvantages: small target mass and relatively large scanning time for each tagged event found in the emulsion.

Two major areas of investigation will be presented in this dissertation. The first will examine aspects of multiparticle production in the sample of neutrino events found in the emulsion. Lepton nucleus interactions are important for studying the development of secondary hadrons. As they traverse the target nucleus, it acts as a detector which is sensitive over lengths of a few fermis. Nuclear emulsion allows a much higher effective atomic weight $\left(A_{\text {eff }} \simeq 80\right.$ ) than other track sensitive detectors. Also, the high spatial resolution of the emulsion permits an investigation of particle production as a function of the number of nuclear fragments (black tracks) and protons (gray tracks).

The second major area in which this dissertation presents results is in the measurement of the lifetimes of charmed particles. Because the weak interaction does not conserve the charm quantum 
number, charmed particles can be produced singly in neutrino interactions. Therefore greater avallable phase space results in a higher rate of charm production ( $5-10 \%$ ) than in hadronic interactions where charmed particles are generally created via associated production (I-2).

The expected decay paths for charmed particles, 10's - 100's of microns, are in a range of values which indlcates that nuclear emulsion is a natural detector to be used to investigate this class of particles. Several earlier experiments have detected charmed particles in emulsion. For example, the first known observation of these particles was in a cosmic ray experiment performed by a Japanese group headed by Niu in 1971. The first hybrid emulsion - spectrometer experiment to see the decay of charm produced in neutrino interactions was performed at FNAL by a group headed by Burhop (I-3).

By investigating the decay times of charmed particles, the basic weak interaction prediction for these times can be verified. Hadronic (possibly Quantum Chromodynamics, QCD) corrections to the decay rates are studied by measuring differences between the lifetimes of the various types of charmed particles. The use of a hybrid detector for this work is essential both for accurate determination of decay times as well as for identification of the parent particle.

Details of this new experimental technique will be presented in chapter II. In chapter III both the theoretical background and the experimental results concerning multiparticle production will be discussed. In chapter IV the current status of charmed meson lifetime studies will be summarized and the contributions of this experiment will be presented. Finally, in chapter V general conclusions that 
can be drawn from these experimental results with respect to the current theoretical framework w1ll be discussed, both for hadron production and for charm decays. 
NOTES TO CHAPTER I

I-1 For a recently completed second exposure of this experiment, the collaboration has been joined by groups from the Illinots Institute of Technology and the University of Sydney.

I-2 M. J. Murtagh, Proceedings of the 1979 International Symposium on Lepton and Photon Interactions at High Energies, Batavia (1979) p. 277.

I-3 see reviews by L. Voyvodic, Proceedings of the 1979 International Symposium on Lepton and Photon Interactions at High Energies, Batavia (1979) P. 569; J. H. Mulvey, invited talk at the Summer Institute on Particle Physics, SLAC (1980). 
CHAPTER II

EXPERIMENTAL PROCEDURES

Exposure at Fermilab

This experiment utilized the wide band neutrino beam produced at FNAL. Each 2 millisecond pulse was the result of an average of $1.6 \times 10^{13}$ protons impinging on an aluminum target. The resulting positively charged particles, largely pions and kaons, were focussed with the 80 kiloamp single horn magnet train before entering the 400 meter vacuum decay pipe. Here the neutrinos were produced in the reactions :

$$
\begin{aligned}
& \pi^{+}+\mu^{+} \nu_{\mu} \\
& \mathbf{k}^{+}+\mu^{+} \nu_{\mu}
\end{aligned}
$$

The muons were removed by the 1000 meter earth and steel berm while the neutrinos reached the experimental area. The resulting spectrum is shown in figure 2-1 (II-1). The average neutrino energy is $25.7 \mathrm{GeV}$. The total exposure for which 315,000 usable bubble chamber pictures were obtained was $5.06 \times 10^{18}$ protons on target. Exposure to this beam yields an expected event rate of 0.024 event / $10^{13}$ protons / ton of target or a total of 850 events expected in this 0.073 ton emulsion target.

The 15-Foot Bubble Chamber filled with liquid deuterium was used as the primary detector for neutrino events occurring in the emulsion target. A 30 kilogauss mangetic field in the chamber provides the sign of the charge as well as the momentum of the charged secondaries. The 3 meter nuclear collision length of the deuterium provides a first 
FIGURE 2-1

Observed neutrino energy spectrum in the 15-Foot Bubble Chamber [II-1]. 


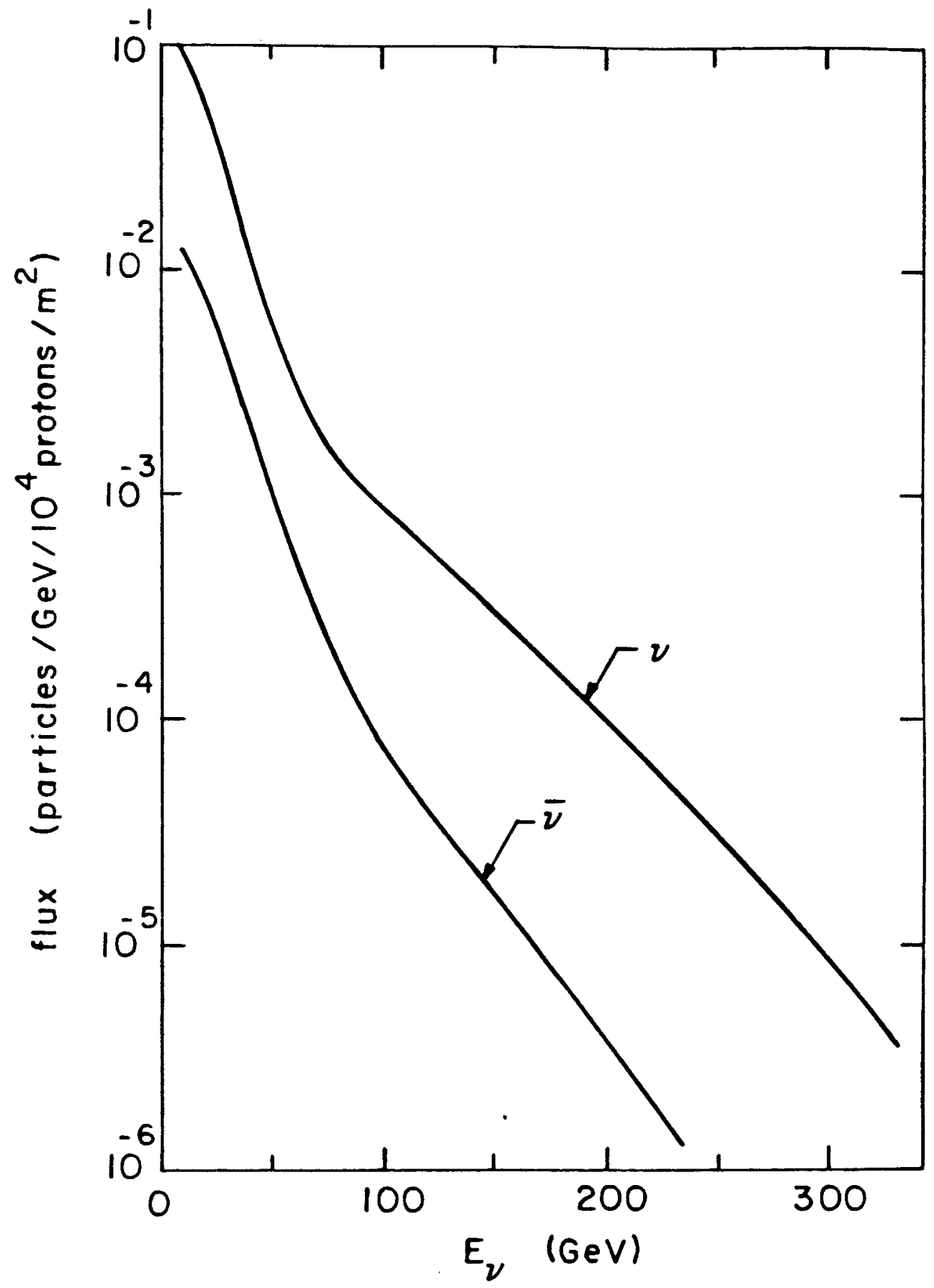


order discrimination between hadrons and muons. The 9 meter radiation length, however, provides low photon conversion efficiency. The long radiation length does have the advantage that a clear view of tracks near the vertex is provided - essential for successful extrapolation of particle trajectories into the emulsion.

Downstream of the bubble chamber following 3 to 5 absorption lengths of shielding or magnet coils are located the 21 chambers of the first plane of the External Muon Identifier (EMI). After 4 to 7 more absorption lengths are located the 18 chambers of the second plane. Tracks which leave the bubble chamber without interacting can be extrapolated to the EMI positions and compared with hits in the multiwire proportional chambers to separate hadrons from muons. Because timing information from each plane is avallable in terms of 35 nanosecond clock counts, accidental hits which are out of time may be eliminated.

Due to curvature in the magnetic field and multiple scattering, the EMI Is utilized only for particles with momentum greater than $4 \mathrm{GeV} / \mathrm{c}$. The configuration of the EMI with respect to the 15-Foot Bubble Chamber and the emulsion target is displayed in figure 2-2.

Emulsion Target

The emulsion track sensitive target for this experiment was assembled from specially manufactured cryogenically sensitive emulsion from the Gos Fotochem Project of the NIKFI Company of Moscow, U.S.S.R. The emulsion was prepared at IHEP, Serpukhov in 22 stacks each consisting of approximately $200-4.6 \times 19.2 \mathrm{~cm}^{2}$ pellicles of nominal 
9

FIGURE 2-2

Location of emulsion boxes with respect to the 15-Foot Bubble Chamber and the EMI. 


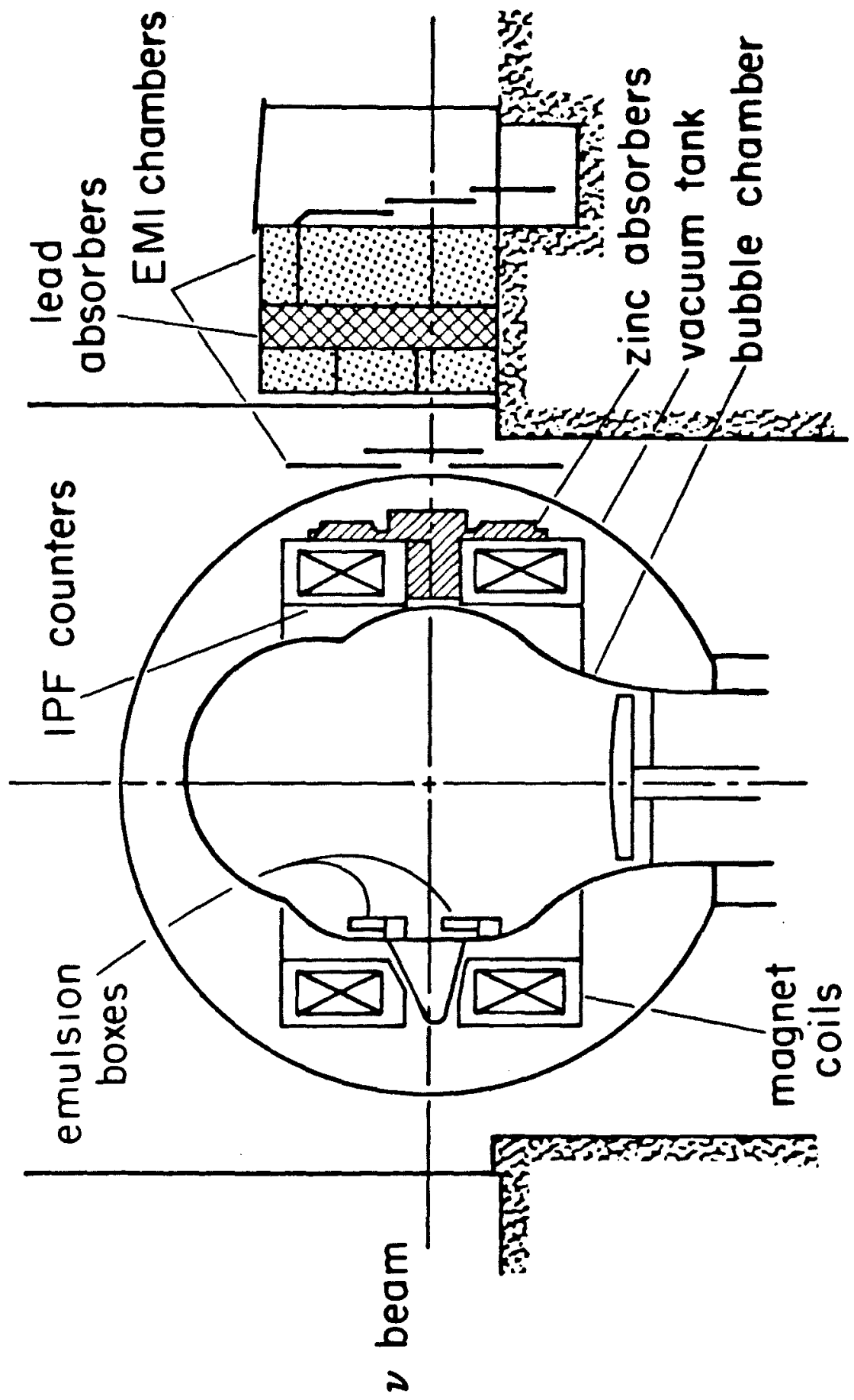


thickness of 450 to 500 microns. The total volume of emulsion was slightly less than 20 liters.

While firmly clamped, the stack faces were milled perpendicular and glued with a gelatin solution to help prevent relative motion between the pellicles. On the front face of each stack was scribed a series of vertical and diagonal lines as shown in figure 2-3. These leave on the edge of each pellicle a pattern of five notches, the spacing of which varies with the location of the pellicle within the stack. In addition to numbering each pellicle and measuring its thickness an independent method of locating the pellicles is thus obtained.

The stacks were wrapped in mylar to prevent water vapor entering or leaving and in black paper to avoid light leaks.

Several factors have influenced the orientation of the stacks with respect fo the beam. An earlier hybrid emulsion-counter experiment performed at Fermilab has shown that multiple scattering limits the efficiency of finding tagged events more than about 5 centimeters into an emulsion stack (II-2).

Preliminary tests for this experiment indicated that errors in predictions of the locations of vertices transverse to the beam direction are smaller by a factor of two in the horizontal direction than the vertical due to better stereo viewing angles and less bubble motion (II-3). Because emulsion scanning within one pellicle is easier than scanning an equivalent volume in several, the stacks were exposed so that the pellicles were parallel with the direction of the larger transverse error. 
11

FIGURE 2-3

Orientation of an emulsion stack and 1ts scribe lines. 


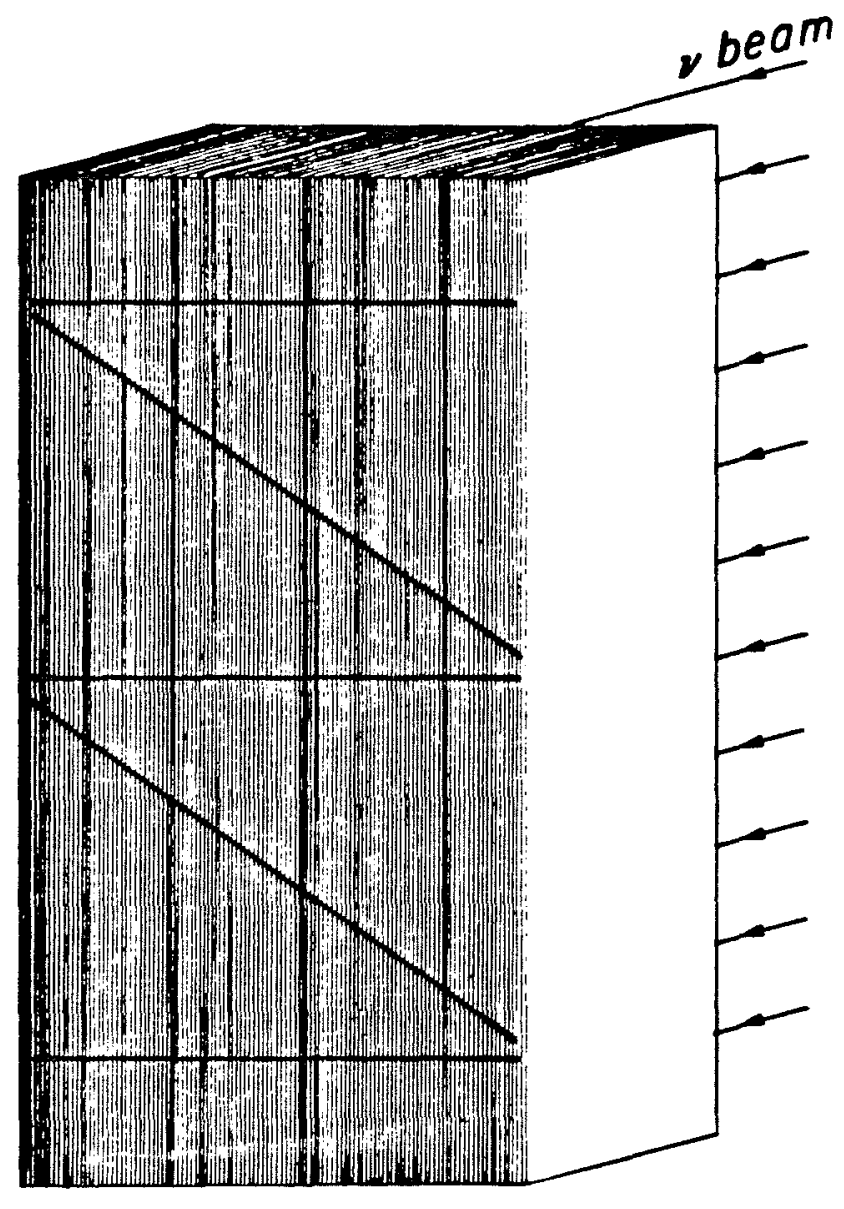


Since the sensitivity of nuclear emulsion decreases as its temperature is decreased below room temperature, special cryogenically sensitive NIKFI BR-2 emulsion was prepared in four separate batches for this experiment. This was accomplished by the elimination of iodine which causes fluorescence at low temperatures (II-4). This also increases the electron trapping efficiency of the silver halide crystals. These trapped electrons act as a prelatent fmage while the conventional latent image is formed when increased temperatures allow greater ionic mobility.

The sensitivity at bubble chamber temperature $\left(30^{\circ} \mathrm{K}\right)$ for these batches has been measured from tagged events to range from $25-30$ blobs / 100 microns.

One result of the low temperature sensitivity is that the emulsion is susceptible to rapid fading at high temperatures. Measurements were performed at Serpukhov on each of the four batches by exposing the emulsion at $+5^{\circ} \mathrm{C}$ to a $50 \mathrm{GeV} / \mathrm{c}$ pion beam and storing the samples for variable lengths of time at $+20^{\circ} \mathrm{C},+5^{\circ} \mathrm{C}$, or $-10^{\circ} \mathrm{C}$ before processing. The results displayed in figure 2-4 show the importance of storing the emulsion at temperatures at or below $+5^{\circ} \mathrm{C}$ following exposure to the neutrino beam. For this reason after being removed from the bubble chamber the emulsion was kept at or below $0^{\circ} \mathrm{C}$ and was sent for processing to the Soviet Union by air while packed in dry ice. Prior to processing at JINR, Dubna, each pellicle had a co-ordinate grid printed on its surface and its thickness measured in three places. Mounting on uniformly sized glass plates was done in a reproducible fashion so that mechanical stops can be used to orfent the 
13

FIGURE 2-4

Fading of tracks in emulsion stored at $+20^{\circ} \mathrm{C},+5^{\circ} \mathrm{C}$ and $-10^{\circ} \mathrm{C}$. 

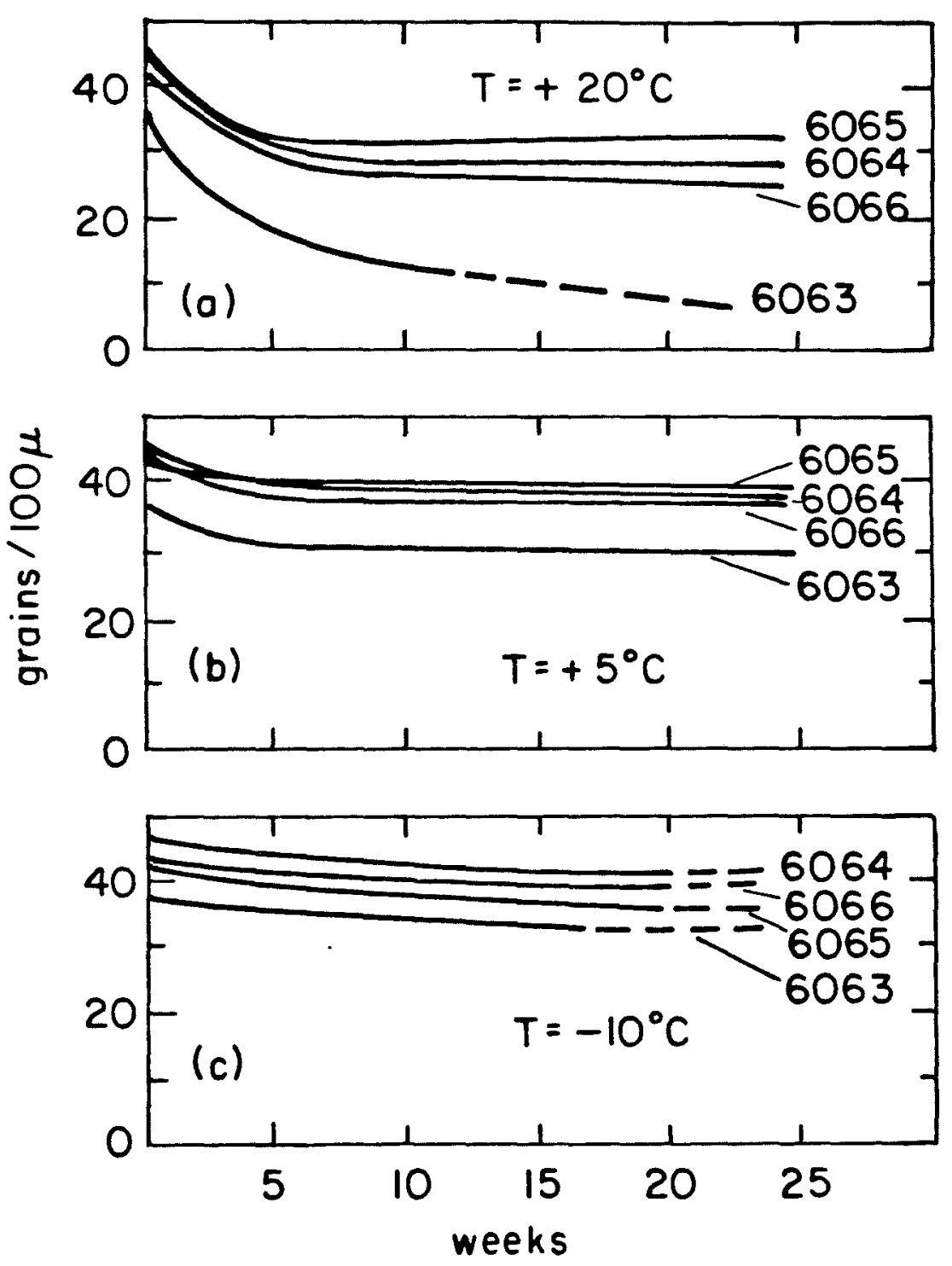
pellicles on a microscope stage accurately and quickly. The preparation and processing of the pellicles at Dubna continued for about three months.

Because the emulsion was exposed at $30^{\circ} \mathrm{K}$ but the measurement of the dimensions of the stacks and the emulsion scanning occur at room temperature, it was necessary to check the thermal contraction of both individual pellicles and a test emulsion stack. The measurements were performed while the emulsion samples were held in a simple cryostat. Distances were measured using a travelling microscope with 8 micron measurement error. Temperatures were measured with an ironconstantan thermocouple and a digital voltmeter.

The fractional change in length from room temperature to liquid nitrogen temperature for the test stack and pellicles was measured to be $(6.8 \pm 0.3) \times 10^{-3}$ and $(7.0 \pm 0.3) \times 10^{-3}$ respectively. This yields a value for the coefficient of thermal contraction for BR-2 emulsion of $(3.2 \pm 0.2) \times 10^{-5} /{ }^{\circ} \mathrm{K}$. The linearity of the contraction for one pellicle to $77^{\circ} \mathrm{K}$ can be seen in figure 2-5. A least-squares fit to the data yields the same value for the coefficient of contraction mentioned above. This data has been utilized in the programs for predicting the locations of tagged events in the emulsion.

To check for hysteresis the emulston was allowed to return to room temperature. The fractional change in the reference length was measured to be $(4.0 \pm 2.5) \times 10^{-4}$ for the test stack.

\section{Mechanical Support}

Two boxes, shown in Plate I, each to hold 11 emulsion stacks, 
15

FIGURE 2-5

Fractional change in length of emulsion pellicle as a function of temperature. 


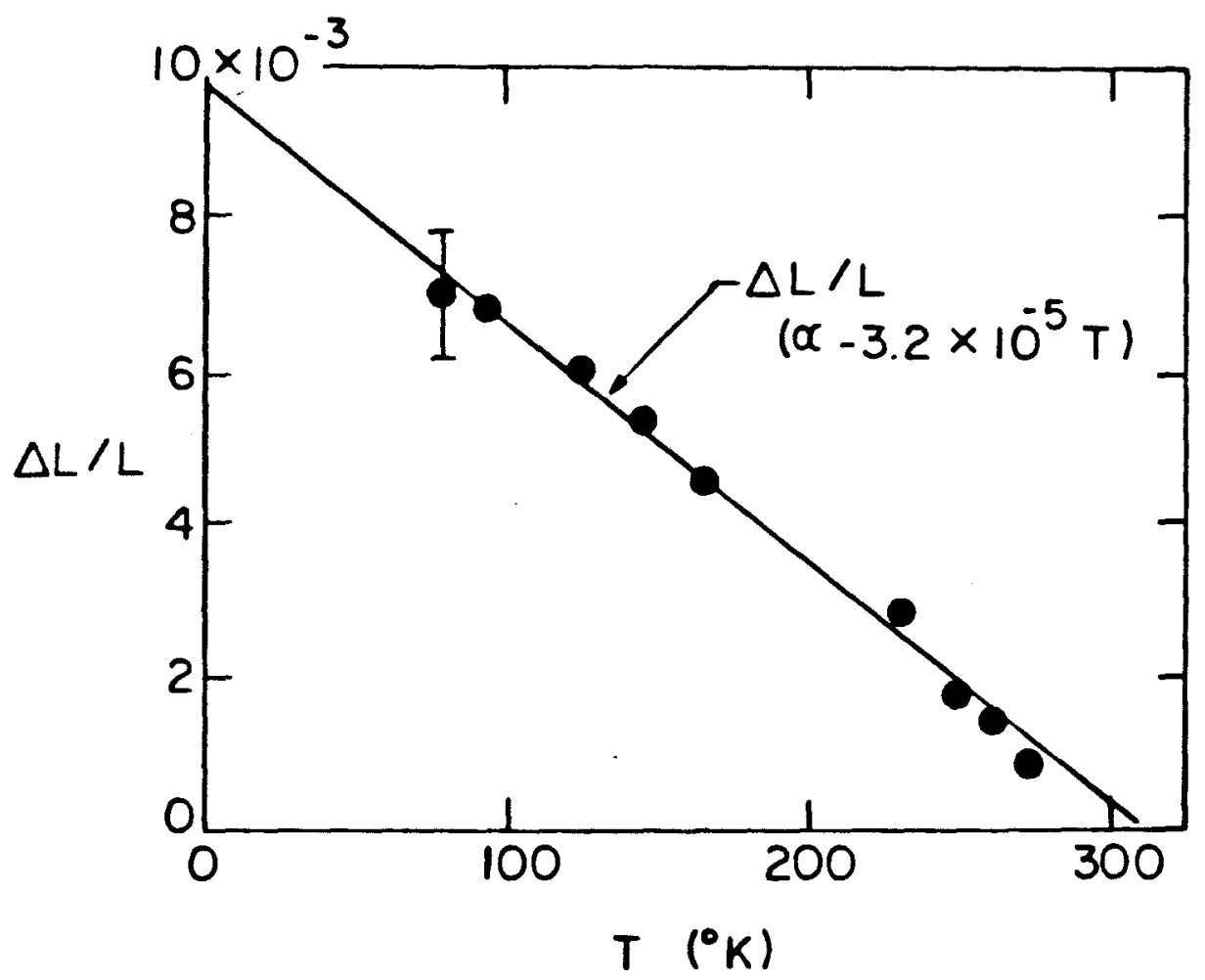


16

PLATE I

Emulston boxes during loading of target. 


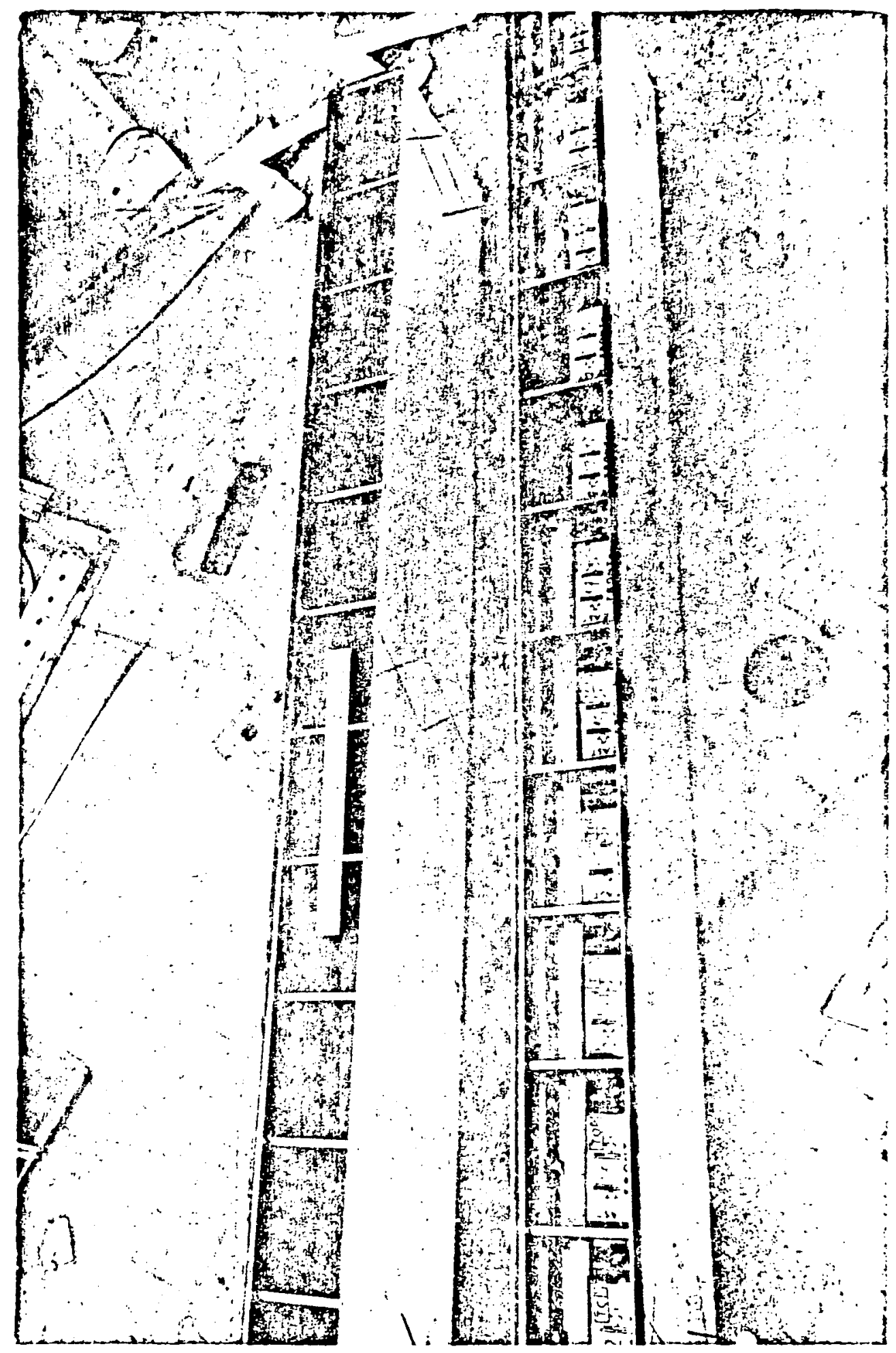


were constructed with nominal outside dimensions of $28 \times 10 \times 152 \mathrm{~cm}^{3}$. One-quarter Inch stainless steel was used for the walls and cell dividers while the base plate was made from one inch stainless steel. The mass of each box $1890 \mathrm{~kg}$.

Two support blocks for each box were welded to the nose cone flange inside the 15-Foot Bubble Chamber. Four bolts through each support block affixed the boxes to the bubble chamber, as 1llustrated in Plate II.

A study of the mechanical vibration of the boxes was performed by Battelle - Pacific Northwest Laboratorles at Richland. The lowest frequency mode is a horizontal vibration at 109 Hertz or greater. The lowest frequency of a vertical mode is expected to be at least 220 Hertz. These are well above the driving frequency of the chamber which is 15 Hertz, so the mode coupling is expected to be small (II-5). Tests performed with the boxes installed in the chamber indicated the estimates of the frequencies to be quite good. For example, the lowest horizontal mode was measured to be at 117 Hertz.

A 10 mil rupture disk was welded over a $1 / 4^{\prime \prime}$ hole into each box interfor. These were to allow a venting of gas while the chamber was returning to room temperature in the event of liquid deuterium leaking into the boxes during the course of the three month run.

Scotchlite was applied to the front face of each box upon which were applied 6 fiducial marks. These fiducials were positioned according to the results of a program designed to give as nearly as possible a perpendicular crossing angle in each of the six camera views (II-6). 
18

PLATE II

Emulsion boxes installed in the 15-Foot Bubble Chamber. 


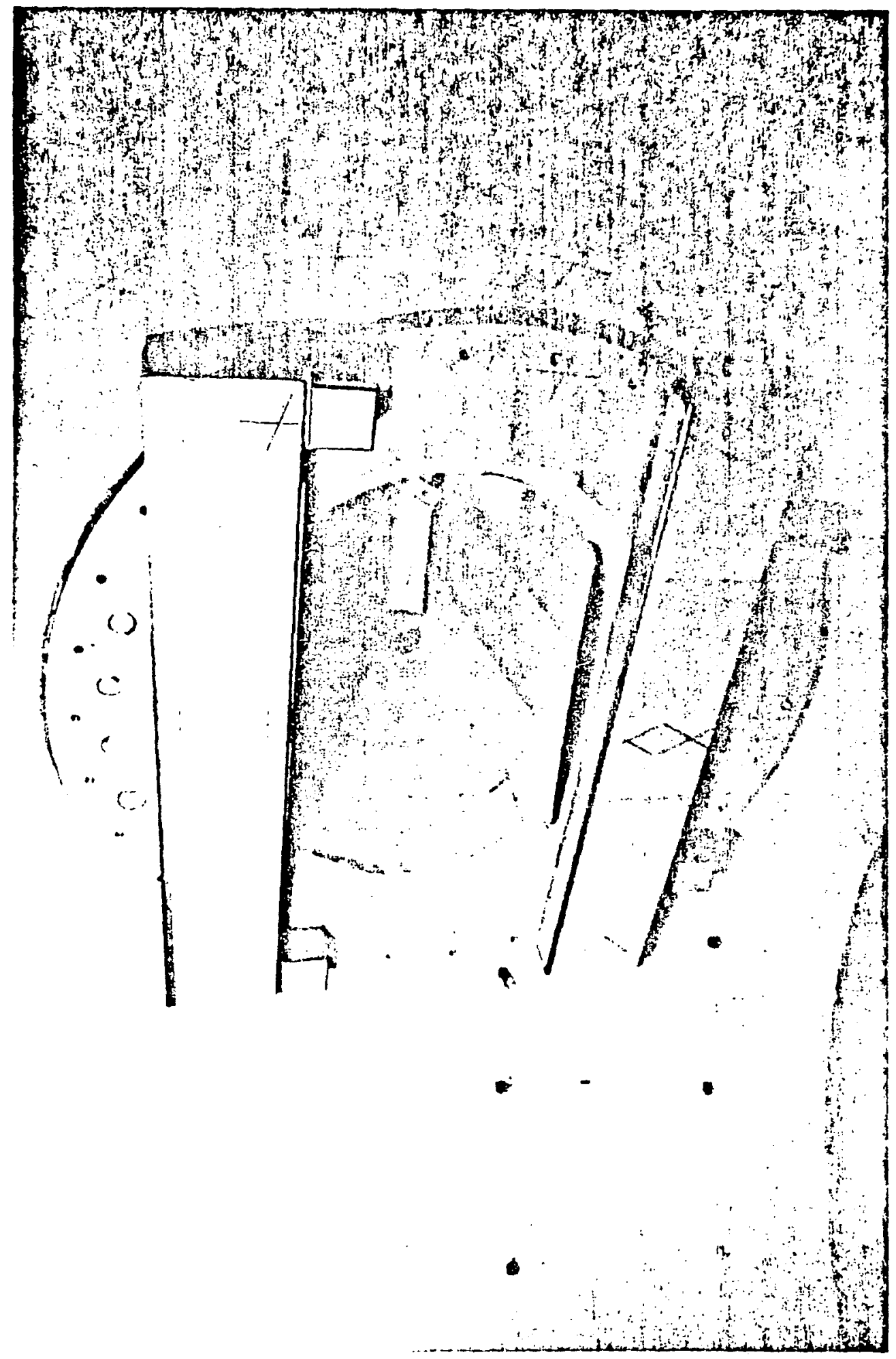


The relative positions of the fiducials and the reference walls of each cell inside the box were established by a survey performed at the FNAL survey shop. The transformation between points inside a box to those outside was done with respect to two punch marks visible on both sides of the stainless steel of the downstream face of the box. The stated errors on these measurements were 50 microns.

Around the outside top edge of each box a notch was milled into which was welded a 1/16" X 1" stainless steel 1ip. After the 22 stacks of emulsion were loaded into the boxes, the $1 / 2 "$ stainless steel lids were welded to these lips to simultaneously seal the boxes while minimizing the heat transfer to the emulsion inside them. To prevent heat build-up in any given part of the boxes, the welding was done in segments no more than $3 "$ long. Also, liquid nitrogen was circulated through copper tubing in contact with the front face of each box to help absorb heat. Thermocouples placed above several stacks and in contact with the box wall indicated that the temperature of the emulsion was not raised above room temperature during the welding process.

The boxes were checked for leaks by using a 1/4" pumping nozzle welded to each. When the vacuum seal was verified, the boxes were back-filled with a nitrogen atmosphere, a gas which is compatible with the requirements of both the preservation of the emulsion and bubble chamber safety.

In order to know the position of each emulsion stack precisely with respect to the three cell walls to be used as reference surfaces, three spring panels have been used to hold each stack firmly in place. 
The convention was adopted to label these panels according to the direction of its normal in the standard Fermllab bubble chamber coordinate system (X - beam direction, $\mathrm{Y}$ - horizontal, $\mathrm{Z}$ - vertical). Several $X$ and $Y$ panels can be seen in place within one of the stainless steel boxes in Plate I.

These panels were constructed in the Physics Department Machine Shop at the University of Washington. The main components of them were 1/4" G-10 and stainless steel springs obtained from the Paragon Spring Company of Chicago. Each X and $Y$ panel contained 8 springs while the $\mathrm{Z}$ panels used 6 . The dimensions of the G-10 pieces were slightly smaller than those of the stack face agalnst which it pressed to guarantee that each panel was in contact with the stack.

Each panel was held together with the springs slightly compressed by a length of $1 / 16^{\prime \prime}$ stainless steel wire rope with a brass hex nut silver soldered to each end. The wire rope was threaded through three holes in a $1 / 4^{\prime \prime}$ stainless steel rod located along the length of the panel. A roll pin through the end of the rod can then be used to turn the rod with a special wrench which then compresses the panel as the wire rope is wound around the rod. A Y-panel being installed by this method is shown in Plate III.

Each panel was calibrated in terms of force versus thickness. Using this information, the thickness of lucite and stainless steel spacers for each was calculated to have each stack held in place with nearly equal force. The average force in each direction with the associated standard deviation is listed below:

$$
X: 180+14 \text { pounds }
$$


21

PLATE III

Installation of an emulsion stack. 


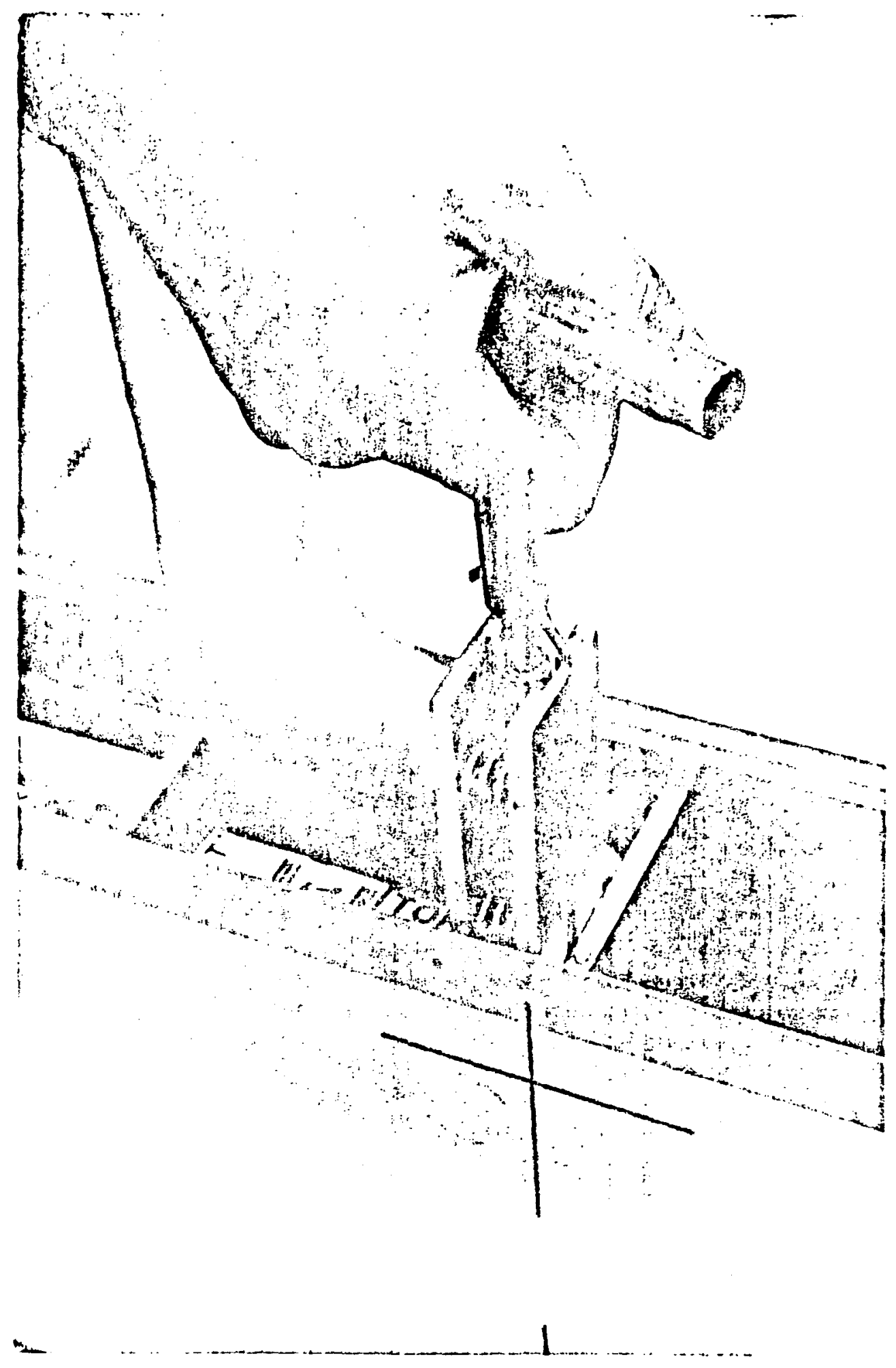




$$
\begin{aligned}
& Y: \quad 140+13 \text { pounds } \\
& Z: \quad 120+10 \text { pounds }
\end{aligned}
$$

Bubble Chamber Film - Scanning and Copying

The 15-Foot Bubble Chamber is equipped with 6 cameras for photographing tracks. Three were used by the primary users, E545 from IIT, Maryland, Stony Brook, Tohoku, and Tufts. For the first 125 rolls of film one camera was allocated for E564 while for the final 55 rolls this was increased to two cameras.

Because at least two views are necessary for stereo reconstruction, a film copying procedure was necessary for all events measured from the initial part of the exposure. For the final portion a preliminary measurement could be performed using two views. Film copying could then be limited to those events which survived an Initial fiducial cut.

During the exposure itself a copying program was carried out with the co-operation of the E545 collaboration to obtain as much film for early measurements as possible. Film was scanned in one view and the roll and frame numbers of all possible events within the emulsion boxes were recorded. Physicists classified each event to be copied on the basis of a possible muon candidate (negative leaving track), presence of hadrons (interaction of secondary particle visible, to eliminate through track plus knock-on electron events) and event location (using templates to eliminate events occurring in the nose cone flange and the stainless steel boxes). Those events which were deemed possibly useful but doubtful were copled once, while 
those more promising were copled twice - one copy for the U.S.S.R. and one for the U.S.

The method of copying which was devised was unique to this experiment and has since been utilized by other groups. Kodak LD-7 Precision Line Film, a high contrast copy film, has several properties which make it useful for this application (II-7). Both the emulsion and the 7 mil Estar base have high dimensional stability. The emulsion is slow enough to be used, with moderate caution, in the ambient light of a bubble chamber scanning room and to permit reasonable exposure times (10 - 30 seconds) on a typical scanning table. Available only in sheet form, 11" X 14" sheets were cut into 8 - $70 \mathrm{X}$ $175 \mathrm{~m}^{2}$ strips. These strips were held in place with respect to the original film (emulsion to emulsion) in felt covered aluminum clamps. Minor modifications were made to one scanning table at Fermilab to facilitate this copying procedure, including the addition of a timing switch to extinguish the lamps of all three views simultaneously after a preset length of time. The length of exposure required was obtained using a commercial enlarger light meter which was calibrated for the scan table. For speed and convenience, the three views of a given roll and frame were copied simultaneously. It was found that the most consistent results were obtained by keeping the lamp voltages constant at the maximum value while correcting for differences in required exposure between the views by using neutral density filters. Small strips of these filters were also used to correct for differences in required exposure across the $70 \mathrm{~mm}$ image of the bubble chamber volume. 
Following the emulsion-to-emulsion exposure, the LD-7 copy of the event was developed in a Kodak Versamat commercial processing machine at Argonne National Laboratory.

Comparisons between measurements using both original and copied film were carried out at Kansas and Seattle. For example, using the results of the Kansas measurements, the values of the average track residuals for the two are the same within errors. A track by track comparison of copied film residual minus original film residual is consistent with being zero.

Bubble Chamber Predictions

Predictions of vertex locations from the Kansas system were based on the TVGP (Three View Geometry Program). Because the vertex cannot be seen in the bubble chamber film, special care was taken to have tracks measured as close as possible to the downstream box face and to have the density of measurement points along the tracks highest near the box to facilitate accurate extrapolation into the emulsion. This extrapolation was performed by the program LOCATE which accounted for the effects of multiple scattering and curvature in the magnetic field. A vertex prediction was obtained by performing a multi-track fit for up to 6 tracks. If one of the tracks failed to pass within an acceptable distance of the predicted vertex location, 1t was rejected and replaced by any remaining unused but measured track for the event. A prediction based on the first 6 acceptable tracks is the result. Any tracks not utilized were used as the basis for a secondary prediction. The location of the vertex 
prediction was first made in terms of a co-ordinate system with its origin in the reference corner of the individual cell of interest. Next a conversion was performed giving the co-ordinates in terms of the pellicle number and photographic grid on each pellicle.

Three methods for converting the cell-space co-ordinate into pellicle number were studied. One simply adds the Individual pellicle thicknesses from the base of the stack until the desired distance is obtained. This method is tedious and has the disadvantage that a correction must be made for the fact that the sum of the individual thicknesses does not exactly equal the thickness of the stack. A second method using the separation of the notches scribed on the face of the stack was utilized as the back-up system. The primary means of determining pellicle number takes each stack to be composed of several groups of pellicles with approximately equal thickness. The sum of each group thickness was adjusted so that the total dimension of the stack equalled the measured value. All three methods gave consistent results within $1 / 2$ pellicle so the third method was adopted due to its ease of use and smaller necessary data base.

Two parallel systems were established in the Soviet Union, both based on the CERN HYDRA package. One from IHEP used a method similar to LOCATE described above in that it performed a multi-track fit. The program from ITEP used a method similar to one described by CERN experiment WAI7 (II-8). Basically, the tracks are extrapolated into the emulsion to a plane perpendicular to the beam direction. A weighted center for the tracks is calculated and a chi-square is 
formed:

$$
x^{2}(x)=\sum_{i}\left(d_{i}^{2} / \sigma_{i}^{2}\right)
$$

where $d_{1}$ is the distance from the center to the track and $\sigma_{1}$ is a function of the track errors in the three spatial dimensions, momentum, azimuth, dip, and the multiple scattering angle. The location of the minimum value of $\chi^{2}(x)$ along the beam direction gives the position of the $\mathrm{X}$ co-ordinate of the vertex. Various track combinations are tried and those for which the probability of the minimum chi-square is greater than $10^{-2}$ are retained.

In order to facilitate the transmission of data and predictions between groups in the Soviet Union and the United States, a Data Exchange Tape (DET) format based on the HYDRA package was used. Similar systems were used by an earlier joint U.S. - U.S.S.R. experiment (FNAL E180) and by FNAL E546, another VTL experiment. In addition to the bubble chamber information contained in earlier DET's, the E564 format also provided for banks for the emulsion data.

Emulsion Scanning

The result of the bubble chamber measurements and extrapolations is a prediction for the event location in terms of pellicle number and co-ordinate grid squares on each pellicle and the associated standard deviations. The average quoted errors for all found events are $3.1 \mathrm{~mm}, 1.5 \mathrm{~mm}$, and 2.2 pellicles for the beam, vertical, and horizontal directions respectively. For those events for which a search was performed, a paralleleplped at least two standard deviations on a side centered on the predicted location was scanned. 
In the case of small quoted errors a standard search volume was adopted which was \pm 6 mm in the beam direction, \pm 1.5 mm in the vertical direction on 6 or 7 pellicles. This gives a total search area of about $240 \mathrm{~mm}^{2}$. The average full time equivalent scanner covers approximately $80 \mathrm{~mm}^{2}$ per day so each searched event takes about 3 full time equivalent scanner-days.

Scanning at Seattle was performed at a magnification of $275 \mathrm{X}$ using $22 \mathrm{X}$ oil immersion objectives. Tests of scanning efficiency using background and cosmic ray events with at least 3 heavily lonizing tracks with $22 \mathrm{X}$ oll versus $10 \mathrm{X}$ air objectives indicated an $88 \%$ efficiency for the former and $58 \%$ for the latter. These results justified the slower search rate which resulted from the higher power objectives. The measured scanning efflciency for events with $N_{h}$ less than 3 falls by at least a factor of two for $22 \mathrm{X}$ ofl scanning while that for $10 \mathrm{X}$ air scanning is near zero.

For the emulsion scanned in Seattle, the average number of background stars found was $0.330 / \mathrm{mm}^{2}$, of which $0.063 / \mathrm{mm}^{2}$ had at least one minimum lonizing track. Rarely did more than one background star per event scan volume have shower tracks in any of the predicted directions and hence warrant a complete measurement. Although the probability of background events masking as tagged events is apparently small, an attempt to quantify the number of mis-identified events was made. Using a subset of the first found neutrino events, an exponential fit to the integral production angle distribution has been obtained:

$$
N(>\theta)=N_{0} \exp \left(-0.043 \theta^{\circ}\right)
$$


Figure 2-6 A and B show the distributions of differences between the predicted and found azimuth and dip angles respectively. Eighty percent of the tagged tracks are within $\pm 2^{\circ}$ of the predicted values for azimuth and dip. Using this information the probability of a background event being mistaken for a tagged event can be calculated. Typically this value for an event with 2 or more minimum lonizing tracks in the forward direction is less than $1 \times 10^{-4}$.

By convention, a track is considered to be tagged if the azimuth and dip each agree within $\pm 2^{\circ}$ of those predicted. An event is considered to be tagged if two or more tracks meet the above criteria. If there is a minimum ionizing track in the upstream direction, the event is called a background hadron event, otherwise it is classified as a tagged neutrino interaction.

A total of 450 events were searched for in the emulsion yielding a finding efficiency of 14\%. Figure 2-7 A, B and C display the differences between the predicted and found locations of the events in the three directions (beam, vertical and pellicle number). Offsets have been included in the prediction programs to eliminate the effects of translations. Figure 2-8 A, B and C show these differences norma1ized to the errors in the respective predictions. Chi-squared tests of two hypotheses - a flat distribution and a Gaussian of width one have been performed and the results shown in Table II-1. The results indicate that with the exception of the vertical direction, the Gaussian distribution hypothesis is favored as expected. Although a significant number of events are lost in the tails of these distributions, the increase in scanning volume to eliminate this loss would 
FIGURE 2-6

Difference between predicted and found track angles. 2-6a is azimuth. 2-6b is dip. 

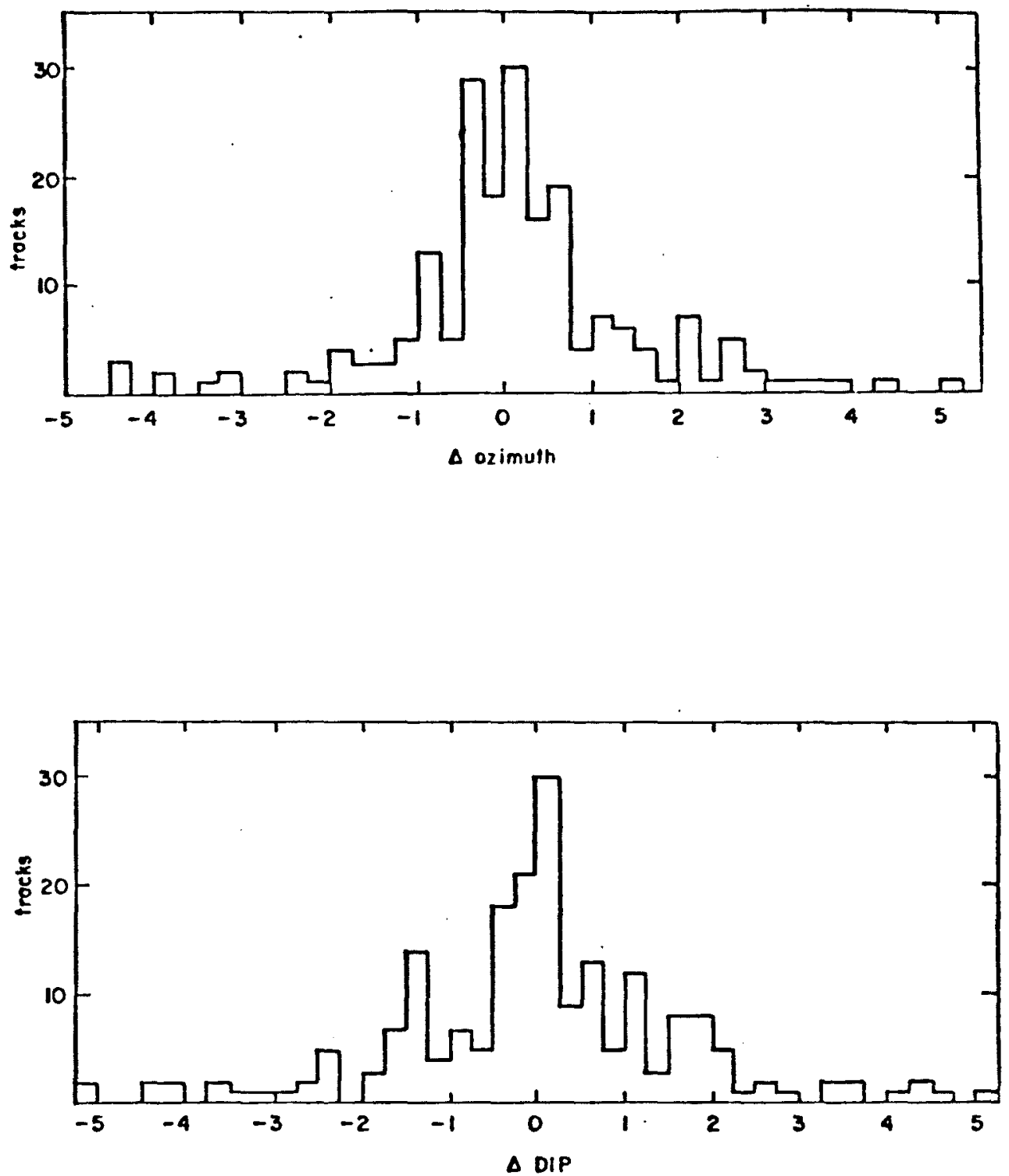
FIGURE 2-7

Difference between predicted and found event locations.

$2-7 a$ is the beam direction. $2-7 b$ is the vertical direction.

$2-7 c$ is the pellicle number. 

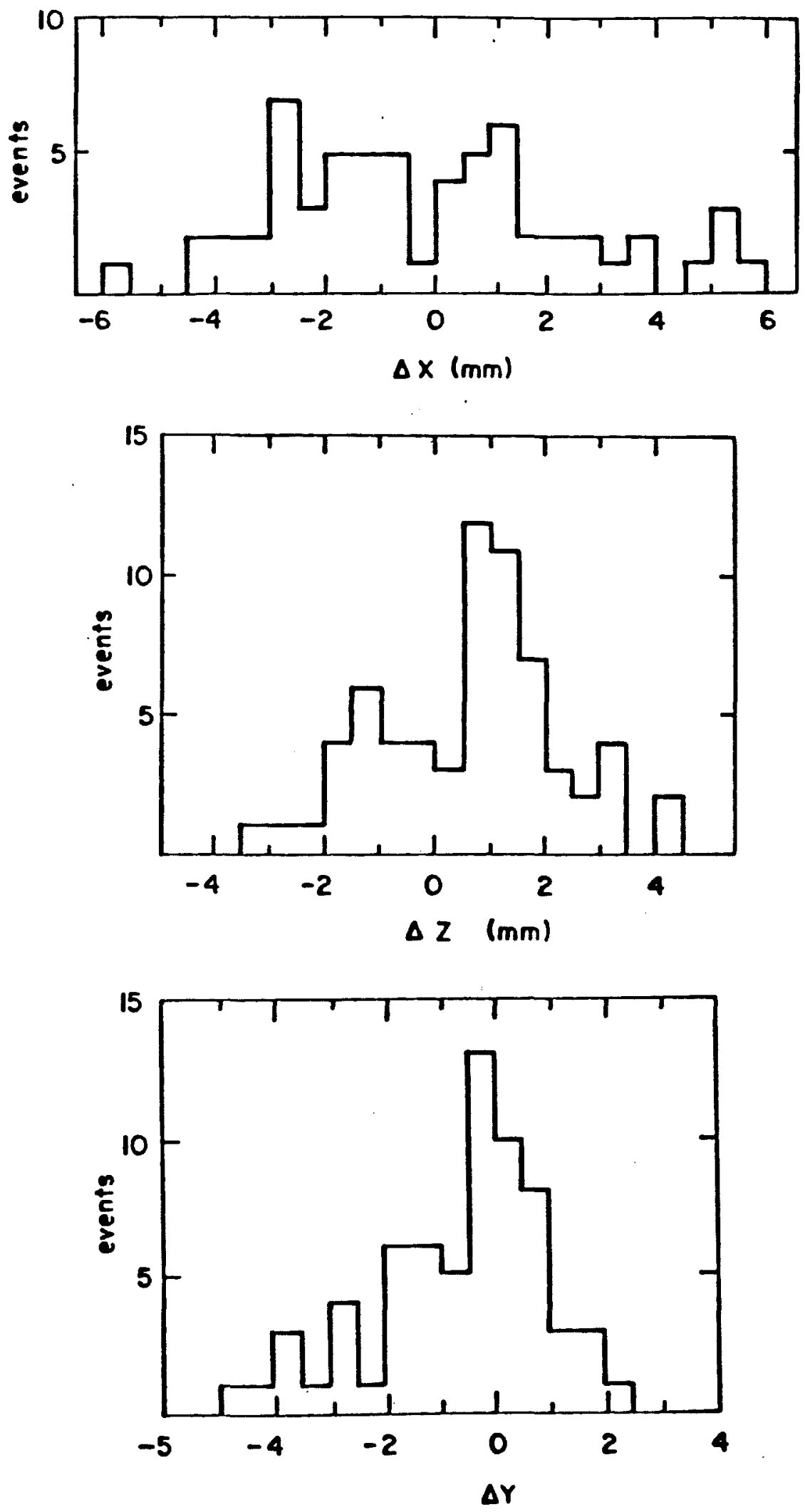
31

FIGURE 2-8

Difference between predicted and found event locations normalized to the quoted error in the prediction. 2-8a is the beam direction. $2-8 b$ is the vertical direction. $2-8 c$ is the pellicle number. 

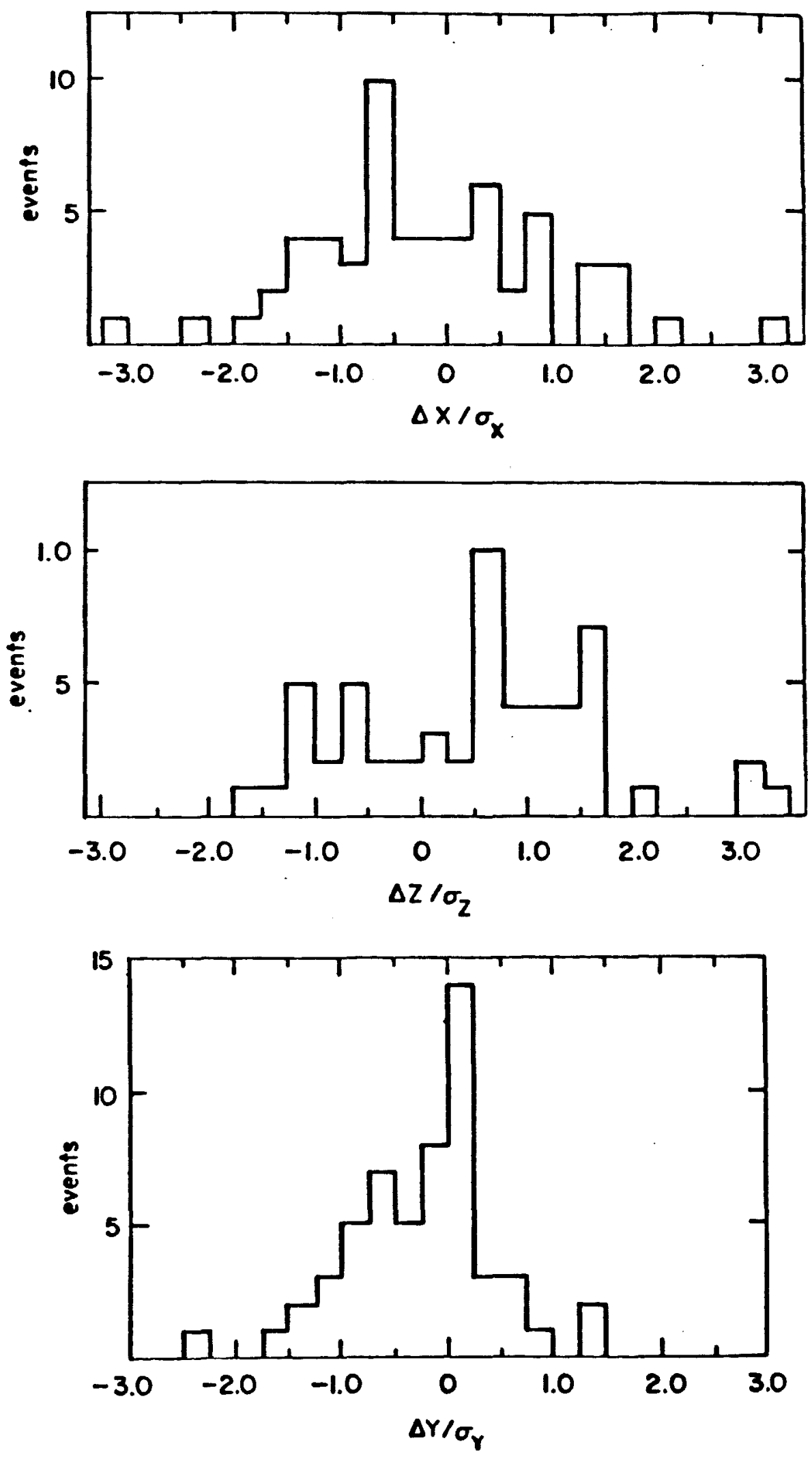
TABLE II-1

Chi-square tests of Gaussian and flat distributions of (predicted-found) locations of events

\begin{tabular}{lcccc}
\hline \multirow{2}{*}{ Direction } & \multicolumn{2}{c}{ Gaussian } & \multicolumn{2}{c}{ Flat } \\
& $\chi^{2} /$ DOF & Probability & $\chi^{2} /$ DOF & Probability \\
\hline Beam & 1.00 & 0.40 & 1.97 & 0.07 \\
Horizontal & 3.20 & 0.01 & 7.03 & $<10^{-4}$ \\
Vertical & 2.58 & 0.03 & 1.45 & 0.20 \\
\hline
\end{tabular}


be prohibitive.

No clustering is observed to occur in the location of found events with respect to the co-ordinates perpendicular to the beam direction. Figure 2-9 shows the distribution of events in the beam direction. Here a large proportion of the events are near the front faces of the boxes while relatively fewer events are found in the upstream part of the emulsion. This is the expected result showing the effects of multiple scattering and a longer lever arm on the accuracy of extrapolating particle trajectories. The finding efficiency for events within $2.5 \mathrm{~cm}$ of the wall of the boxes is $20 \%$ while that for emulsion more than $2.5 \mathrm{~cm}$ from the wall is $11 \%$.

Figure 2-10 displays the integral distribution of all events found with respect to the number of heavily ionizing tracks (tracks with $\mathrm{dE} / \mathrm{dx}$ greater than 1.4 times the minimum value of $\mathrm{dE} / \mathrm{dx}$ ). The dashed line shows this distribution from a hybrid emulsion-counter experiment performed at FNAL, E531, which used a track following technique and hence avoided any scanning bias (II-9). The two curves have been normalized to equal area for $N_{h}$ greater than 3 . It is observed that scanning losses are large for events with a value of $\mathrm{N}_{h}$ less than 3. However, as will be justified later, this effect is not thought to introduce a significant kinematic bias.

For each neutrino event found in emulsion a search was performed for possible charmed particle decays. For neutral decays a volume scan region was defined by a $45^{\circ}$ half-angle cone. A high power scan (1250X) was performed for the first 500 microns downstream and a somewhat lower power scan $(660 \mathrm{X})$ was continued for a total of $2 \mathrm{~mm}$. 
34

FIGURE 2-9

Distribution of the event locations in the beam direction 1.e., depth in emulsion. 


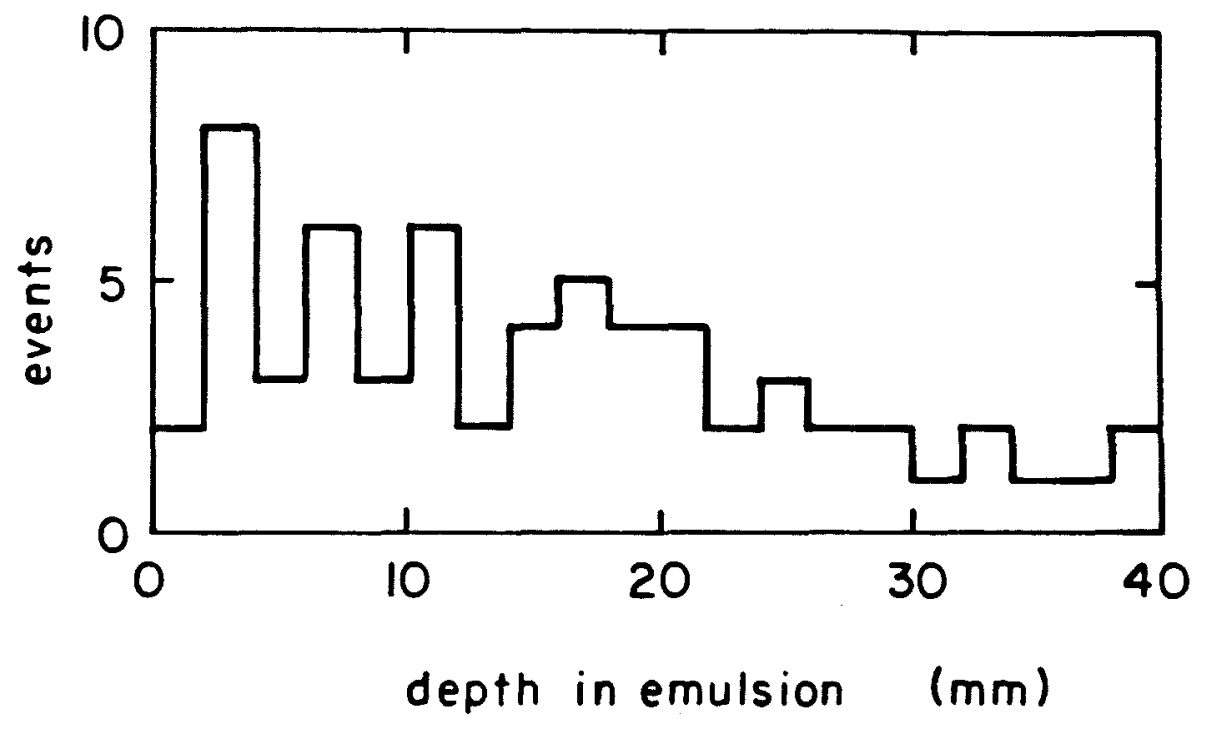


35

\section{FIGURE 2-10}

Distribution of the number of heavily lonizing tracks. Results from this experiment are crosshatched. Results from reference [II-9] are also shown. 


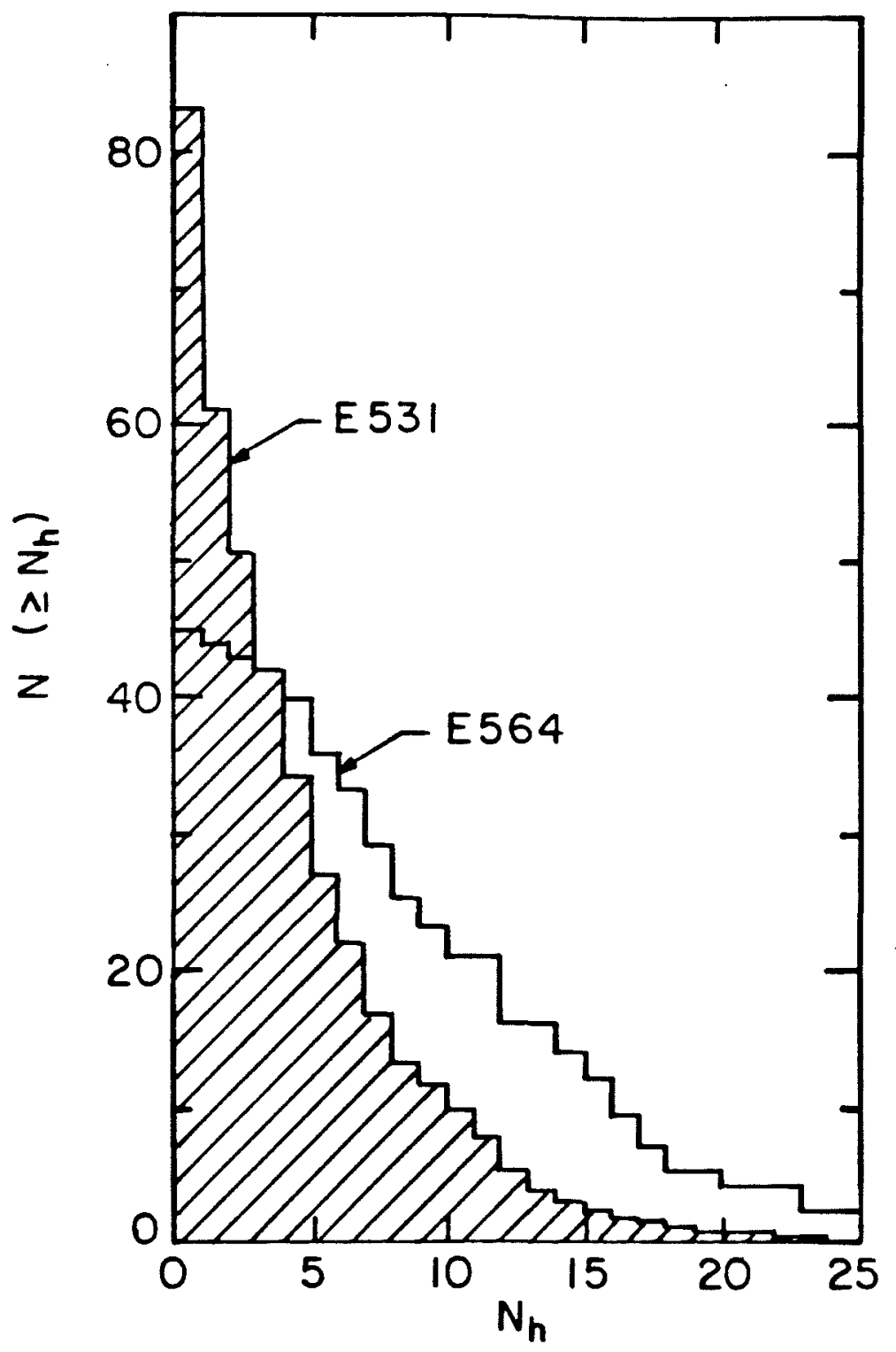


All minimum and gray tracks were followed for a distance of at least $3 \mathrm{~mm}$. Generally these were followed as far as practicable. The 10cation of any vee, kink or other apparent decay was recorded for further investigation. 
NOTES TO CHAPTER II

II-1 J. Hanlon, private communication.

II-2 A. L. Read et al, Phys. Rev. D19, 1287 (1979).

II-3 L. Voyvodic et al., Fermilab proposal P564 (1977).

II-4 C. S. Bogomolov et al., Proceedings of the Tenth International Conference on Solld State Nuclear Track Detectors, Lyon (1979) p. 127.

II-5 F. A. Simonen, private communication.

II-6 W. Smart, FNAL TM-795 (1978).

II-7 KODAK, KP 73555 1-77 (1977).

II-8 P. Bagnaia et al., University of Rome Internal Note N. 706 (1978).

II-9 K. Niu, Proceedings of the Twentieth International Conference on High Energy Physics, Madison (1980) p. 352. 
CHAPTER III

MULTIPARTICLE PRODUCTION

Theory and Background

Multiparticle production in hadron - nucleus interactions has been used as a tool to study the space-time development of the final state hadrons over a distance of order of the nuclear size. More recently lepton - nucleus scattering has been investigated for the same purpose, making use of the simple structure of the leptonic vertex. Due to small lepton - nucleon cross sections, the lepton scatters only once in the nucleus. In the case of charged current neutrino events the outgoing muon does not rescatter. Thus, as discussed by Bialas and Czyz, any increase in multiplicity with Increasing atomic weight of the target for these cases demonstrates the existence of intranuclear cascading (III-1). Such an increase in the multiplicity in the target fragmentation region has been observed, for example, in neutrino - neon interactions and in muon - emulsion interactions (III-2 and III-3).

Several authors have presented models which predict characteristics of neutrino - nucleus interactions which are accessible to examination with respect to the data from this experiment. In particular this experiment allows the determination of normalized multiplicity and angular distributions for interactions with a much heavier atomic target than standard bubble chamber experiments. Also because of the high resolution of the emulsion detector, the dependence of these quantities on varying nuclear excltation can be studied. 
Spectific predictions can be tested from the Modifled Cascade Model (MCM) of Hossain and Tow, the Coherent Tube Model (CTM) of Afek, Berland, Dar and E1lam, and the Growth of Longitudinal Distances Model (GLD) of Nikolaev and Davidenko.

Modified Cascade Model

The Modified Cascade Model is a multiple scattering model in which the secondary particles can interact within the nucleus (III-4). Immediately following production they are in an immature state, i.e. they have small inelastic cross sections. After a characteristic time the secondaries become spontaneously mature and hence have the normal particle - nucleon cross section. This process takes place more quickly in the presence of nuclear matter, 1.e. Induced maturity.

The model has one parameter, the mean induced maturity path length which has been fixed on the basis of $200 \mathrm{GeV}$ proton - nucleus data.

Averaging over all impact parameters for a large nucleus yields an average of $\left(0.8 \times \mathrm{A}^{1 / 3}\right)$ nucleons which can interact with the incident particle. This value is independent of the identity of the incident particle.

Input to the model consists of the measured neutrino - nucleon rapidity distribution. Each of the products of the initial interaction is then propagated through the nucleus with an appropriate probability of participating in a secondary interaction. Measured hadron - nucleon rapidity distributions are then used to generate the products of any such secondary interaction. The result after all particles 
have been followed from the nucleus is the neutrino - nucleus rapidity distribution.

Coherent Tube Model

The Coherent Tube Model assumes that the $W$ boson emitted by the neutrino strikes a parton in the nucleus which is part of a tube of nucleons (III-5). The tube has the diameter of a nucleon and extends across the nucleus. Thus the target mass is the summed mass of the entire tube. This means that a projectile with energy $v$ will produce a center of mass squared-energy of $w^{2}=2 M_{N} 1 v$ where 1 is the number of nucleons in the tube. For a large nucleus the average value of $i$ is approximately $A^{1 B}$.

The model also assumes that at the same $w^{2}$ value, an interaction will appear the same whether the projectile strikes a tube or a free nucleon. Therefore the CTM predicts for the multiplicity of neutrino nucleus collisions:

$$
\left\langle n_{c}\left(W^{2}\right)\right\rangle_{V A} \simeq\left\langle n_{c}\left(A^{2 / 3} W^{2}\right)\right\rangle_{\nu p}
$$

The CTM also predicts that distributions in rapidity, $y$, for nucleus interactions at $\mathrm{W}$ and $\mathrm{y}$ should equal distributions for nucleon interactions at $W_{\text {eff }}^{2}=A^{1 / 3} W^{2}$ and $y_{\text {eff }}=y+(1 / 3)(\ln A)$. This prediction is approximately independent of the type of projectile.

Growth of Longitudinal Distances Model

Nikolaev's model is a parton model in which secondary hadrons of momentum $k$ are formed a distance $1_{f} \sim k / m_{0}{ }^{2}$ from the inftial interaction $(I I I-6)$. He takes $m_{0}{ }^{2}$ to be $0.7(\mathrm{GeV})^{2}$ for constituent quarks (III-7). This distance can be understood using the following 
argument. By the uncertainty principle the distance over which a particle can interact is inversely proportional to 1ts mass. Multiplying by a factor for time dilation gives the distance as the energy of the particle divided by the square of its mass. Taking $R_{N}$ as the nuclear radius, those secondaries with $k<\mathrm{R}_{\mathrm{N}} \mathrm{m}_{0}{ }^{2}$ are formed $\mathrm{in}$ the nucleus and contribute to the cascade. Those with $k>R_{N} m_{0}^{2}$ are formed outside the nucleus and cannot rescatter.

A second distance scale enters this model which is $\Delta Z$, the distance over which the virtual $W$ interacts with the nucleus and is given by $\Delta Z \sim v / Q^{2}$. Here $\nu$ is the energy transferred to the hadronic vertex and $Q^{2}$ is the square of the four-momentum transferred. Two extreme cases for the range of $\Delta Z$ exist. If $\Delta Z \ll R_{N}$, then the interaction takes place incoherently and the $W$ is absorbed by nucleon in the volume of the nucleus. The nuclear cross section is just A times the nucleon cross section. On the other hand, the interaction will proceed diffractively and the $W$ will be absorbed on the surface of the nucleus if $\Delta Z>R_{N}$. On the average the cascade has twice as much distance in which to develop in the second case and so the multiplicity is expected to be greater. In this experiment essentially all of the events are in the kinematic region where $\Delta \mathrm{Z} \ll \mathrm{R}_{\mathrm{N}}$.

The model predicts the ratio of rapidity distributions,

$$
R_{y}=\left|\frac{1}{\sigma} \frac{d \sigma}{d y}\right|_{A} /\left(\frac{1}{\sigma} \frac{d \sigma}{d y}\right)_{p}
$$

has a power law dependence on the atomic weight of the target:

$$
R_{y}=A^{\alpha}
$$

where $\alpha$ is a function of rapidity. The intranuclear cascade is 
produced in the central and backward reglons where $\alpha$ 1s greater than zero. In the forward region is a depletion $(\alpha<0)$ due to energy momentum conservation.

Events Found

The results presented here are based on a data sample of 65 tagged events. Because no upstream veto was used, 20 of the events found were produced by incident background hadrons. Figure 3-1 shows the distribution of $n_{s}$, the number of minimum lonizing tracks $(d E / d x$ less than 1.4 times the minimum $\mathrm{dE} / \mathrm{dx}$ ) for the resulting sample of 45 neutrino events. The average value of the multiplictty is:

$$
<n_{s}>=6.78 \pm 0.49
$$

This multiplicity includes the outgoing muon for the charged current events. For the neutral eurrent events all tracks are caused by hadrons. Only multiplicity data and not track angles are avallable for six of the neutrino events.

Muon Selection and Charged Current Cut

For this data sample the muon has been selected by taking the negative track with the greatest transverse momentum with respect to the beam direction (III-8). According to Monte Carlo calculations of Chapman et al., and verified by tests using the EMI, this method selects the muon correctly in $95 \%$ of all charged current events. Due to this high confidence level, the EMI has only been used for special events.

In order to separate charged and neutral current events, a 
43

FIGURE 3-1

Distribution of minimum lonfzing tracks. 


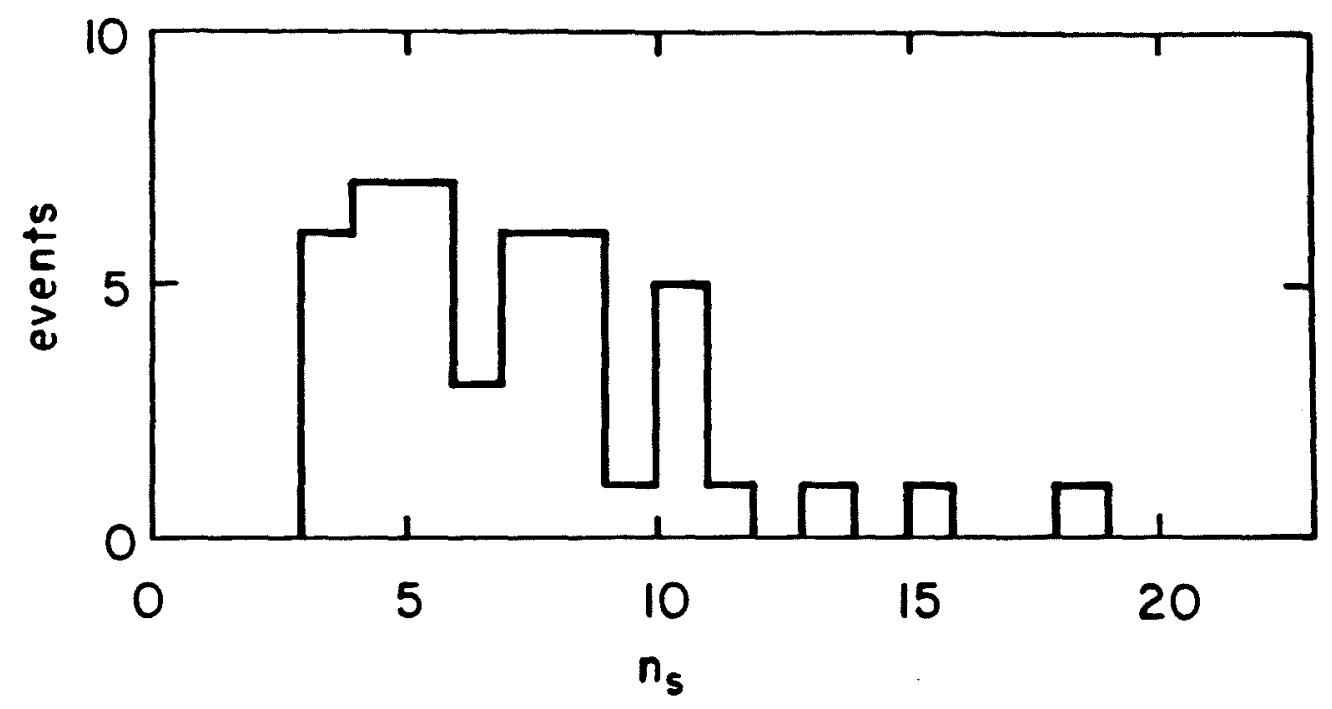


kinematic method used by Fermilab experfment E545 has been adopted (III-9). The quantity $P_{T R}$ is defined as the length of the component of $\vec{p}$ perpendicular to $\vec{p}$. $\vec{p}$ is the apparent muon momentum as selected by the method previously mentioned and $\vec{p}^{\text {h }}$ is the summed momenta of the other charged particles.

Events with $P_{T R}$ less than $1 \mathrm{GeV} / \mathrm{c}$ are labelled neutral current events. Twenty-eight events survive this cut and serve as the charged current sample. Figure 3-2 shows the integral distribution of $P_{T R}$. A linear least-squares fit over the range $1 \mathrm{GeV} / \mathrm{c}<\mathrm{P}_{\mathrm{TR}}<5 \mathrm{GeV} / \mathrm{c}$ of the differential distribution indicates that $6.0 \pm 2.3$ charged current events have been lost in this cut. Monte Carlo calculations performed by E545 to study this method indicate that less than five percent of the surviving events are due to neutral current interactions (III-9).

\section{Energy Correction}

In order to correct for neutral hadrons an energy correction due to Heilmann has been used (III-10):

$$
E_{v}=p_{1}^{\mu}+p_{1}^{h}+p_{1}^{h}\left[\frac{\left|p_{t}^{\mu}+\Sigma p_{t}^{\mu}\right|}{\Sigma\left|p_{t}^{h}\right|}\right]
$$

Here $p_{1}^{\mu}\left(p_{1}^{t}\right)$ is the longitudinal momentum of the muon (transverse momentum of a hadron).

Monte Carlo calculations indicate that the rms difference between the corrected and true neutrino energy is 15\% (III-11). Figure 3-3 gives the distributions of visible and corrected energies. The average visible energy is $26.8 \mathrm{GeV}$ which can be compared with the visible energy distribution for neutrino - deuterium interactions 
45

FIGURE 3-2

Integral distribution of the quantity $\mathrm{P}_{\mathrm{TR}}$. 


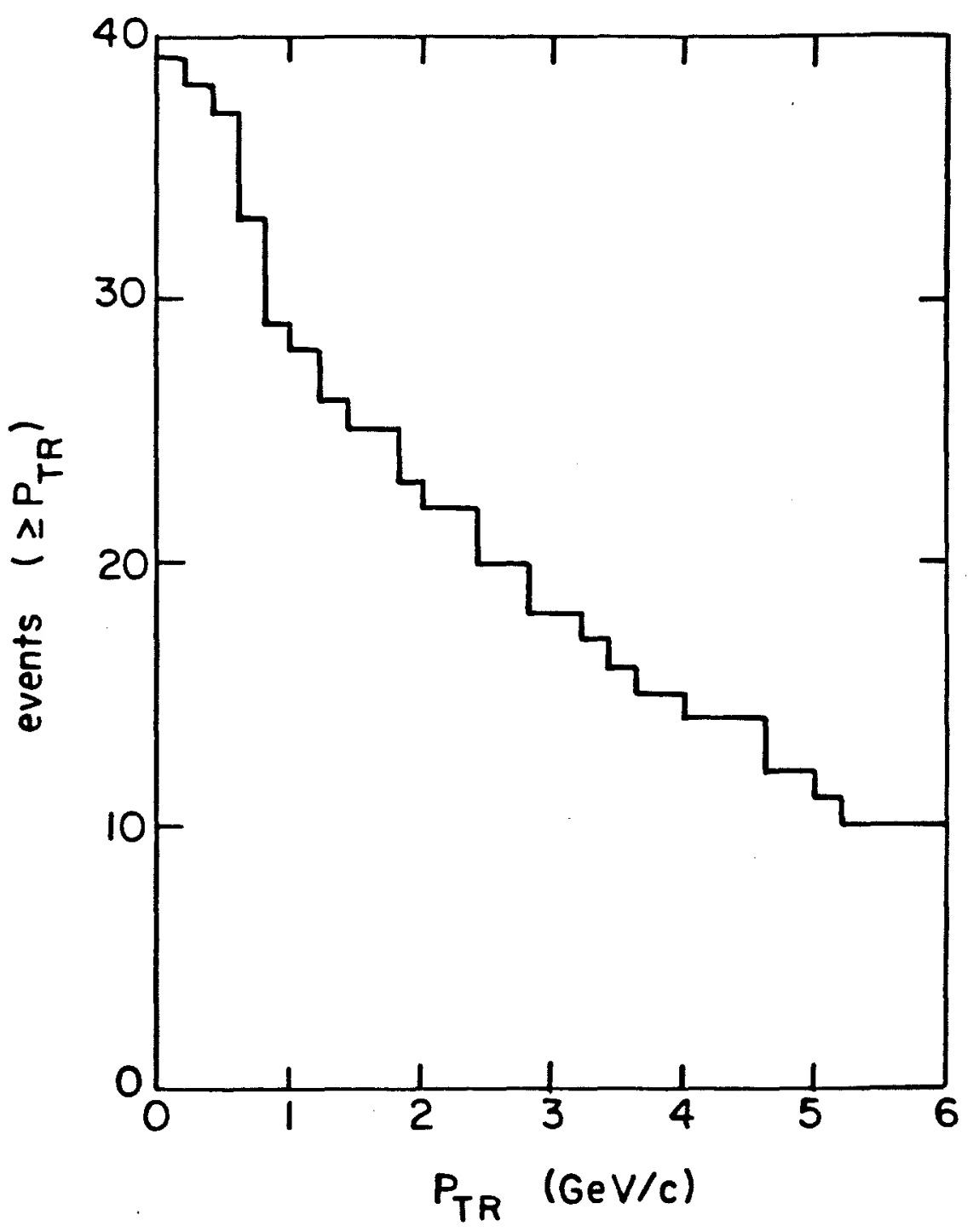


46

FIGURE 3-3

Visible and corrected energy probability distributions.

The visible distribution is crosshatched. 


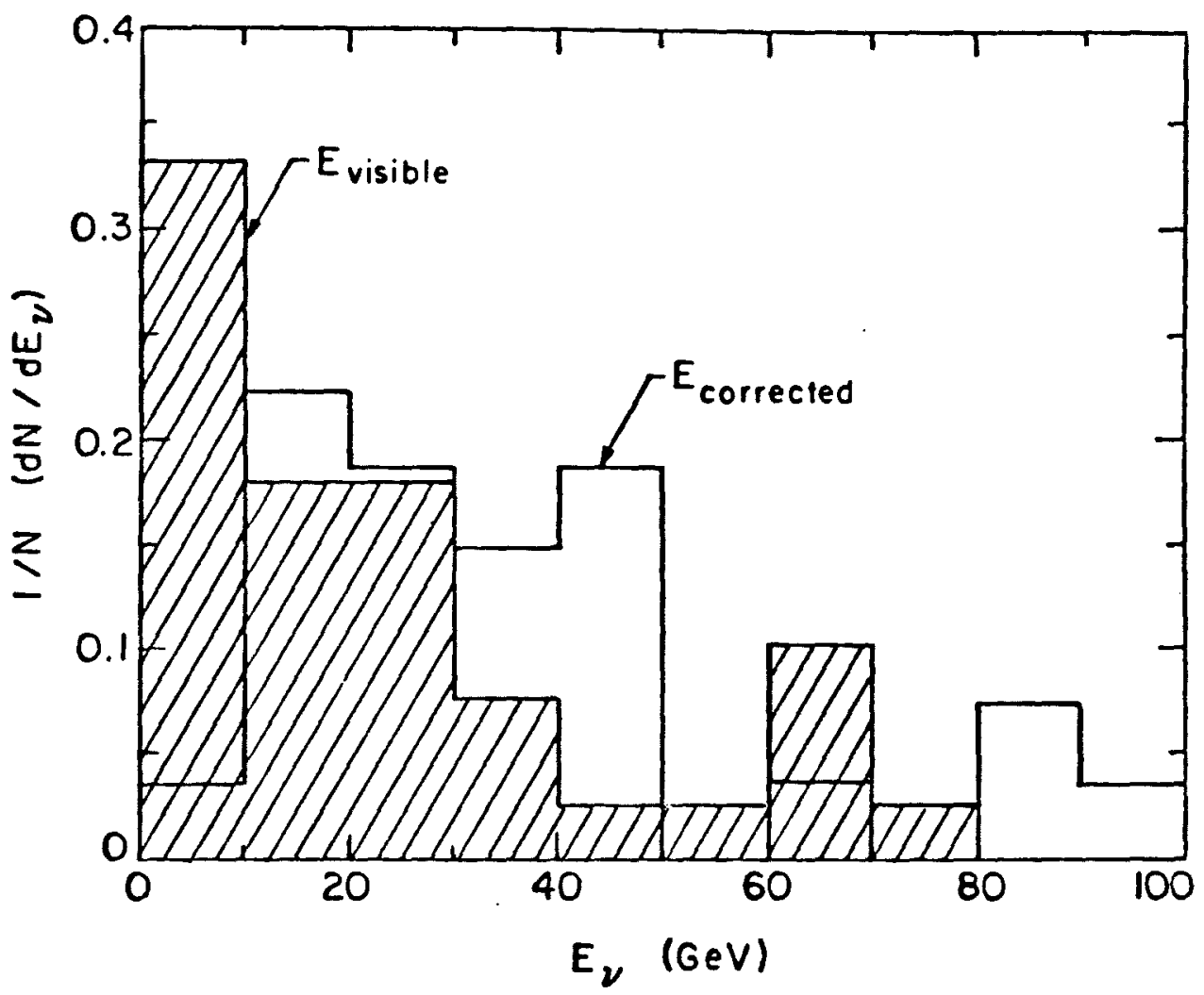


shown above in figure 2-1 which has an average value of $25.7 \mathrm{GeV}$. The average corrected energy for the charged current neutrino - emulsion interactions is $43.8 \mathrm{GeV}$. The average of the ratio between corrected and visible energy on an event by event basis 1s 1.32. This factor is somewhat higher than that reported in other experiments due to tracks which either falled to reach the bubble chamber or were not measured there. Also the poor information on neutral hadrons because of the long radiation length of the liquid deuterium has contributed to the large correction factor.

The distributions for invariant hadronic mass, $W$, and square of the four-momentum transfer, $Q^{2}$, are shown in figure 3-4. These values have been calculated using the relations below:

$$
\begin{aligned}
& Q^{2}=4 E_{\nu} E_{\mu} \sin ^{2}(\theta / 2) \\
& W^{2}=-Q^{2}+2 M_{N} \nu+M_{N}^{2} \\
& M_{N}=\text { nucleon mass } \\
& \nu=E_{\mu}-E_{\nu} \\
& \theta=\text { neutrino - muon scattering angle }
\end{aligned}
$$

The range of $W$ is between 1 and $14 \mathrm{GeV}$ with an average value of 4.8 GeV. Eighty percent of the events are contained in the interval $\mathrm{Q}^{2}<16(\mathrm{GeV} / \mathrm{c})^{2}$ and have an average value of $5.64 \pm 0.88(\mathrm{GeV} / \mathrm{c})^{2}$.

\section{Scanning Blas Test}

Because minimum Ionization tracks are difficult to see at the magnification used for scanning, finding these neutrino events is dependent upon the number of heavily lonizing tracks. As shown in chapter II, a bias against low $N_{h}$ events exists. Therefore, to test 


\section{FIGURE 3-4}

Kinematic distributions. 3-4a is the center of mass hadronic energy, $W$. $3-4 \mathrm{~b}$ is the square of the four-momentum transferred, $Q^{2}$. 

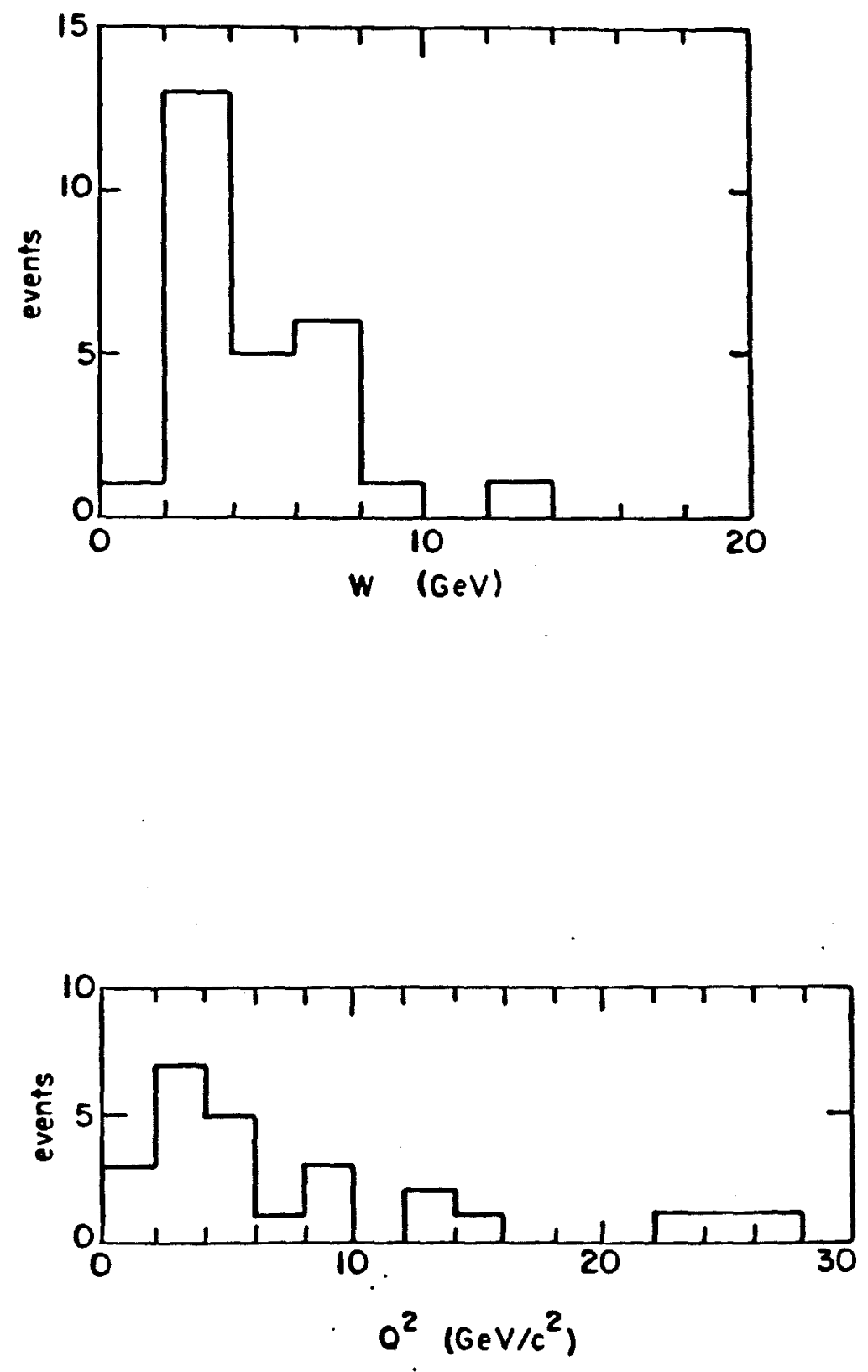
for possible scanning bias effects in these results, the dependence of $\left\langle n_{s}\right\rangle$ and $\langle W\rangle$ on $N_{h}$ have been plotted in figures 3-5 and 3-6. A linear least-squares fit to $\left\langle n_{s}\right\rangle$ versus $N_{h}$ ylelds the result:

$$
\left\langle\mathrm{n}_{s}\right\rangle=(0.15 \pm 0.15) \mathrm{N}_{h}+(6.16 \pm 1.34)
$$

The slope is consistent with zero and any scanning effect is expected to be small. The value of $\langle W\rangle 16$ seen to be independent of $N_{h}$, indicating no correlation between the hadronic center of mass energy and the number of nuclear fragments. Therefore, the known scanning bias is not expected to result in the introduction of a kinematic bias to the data.

Multiplicity Distributions

The average charged hadronic multiplicity, $\left\langle n_{c}\right\rangle=\left\langle n_{s}-1\right\rangle$, for charged current events is seen to rise linearly with the logarithm of $\mathrm{W}^{2}$ in figure $3-7$.

$$
\left\langle\mathrm{n}_{\mathrm{c}}\right\rangle=(1.60 \pm 0.59) 1 \mathrm{n}\left\langle\mathrm{w}^{2}\right\rangle+(1.50 \pm 1.31)
$$

Both the slope and the intercept are consistent with results reported for neutrino - proton interactions (III-12):

$$
\left\langle n_{c}\right\rangle=(1.38 \pm 0.03) 1 n\left\langle W^{2}\right\rangle+(0.38 \pm 0.07)
$$

other experiments have shown that $W$ is the most useful variable in this case for interactions with protons (III-13); however, low statistics prevent a clear separation between $Q^{2}$ and $W$ dependence from this sample. For example, a linear fit of $\left\langle n_{c}\right\rangle$ versus $\ln Q^{2}$ yields the result:

$$
\left\langle n_{c}\right\rangle=(1.46 \pm 0.57) 1 n\left\langle Q^{2}\right\rangle+(2.92 \pm 1.04)
$$

with a $x^{2}$ value of $2.07 /$ DOF which is somewhat higher than that for 
FIGURE 3-5

Average number of shower tracks, ( $n_{0}$ ), versus number of heavily lonizing tracks, $N_{h}$. Also shown is a least-squares fit to the data. 


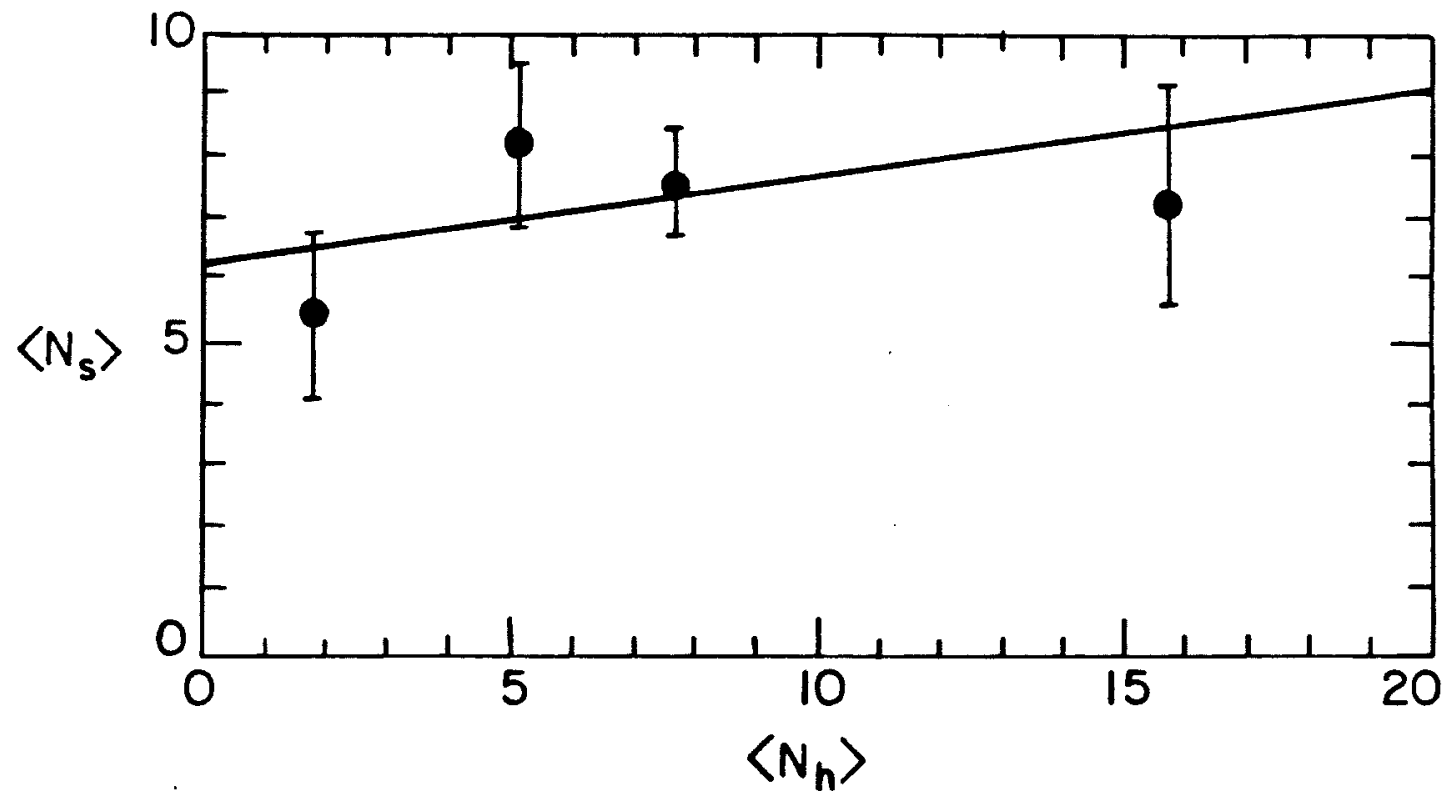


51

FIGURE 3-6

Average center of mass hadronic energy, (W), versus number of heavily lonizing tracks, $\mathrm{N}_{h}$. 


$$
+7
$$


52

FIGURE 3-7

Average number of hadrons, $\left(n_{c}\right)$, versus natural logarithm of $w^{2}$. 


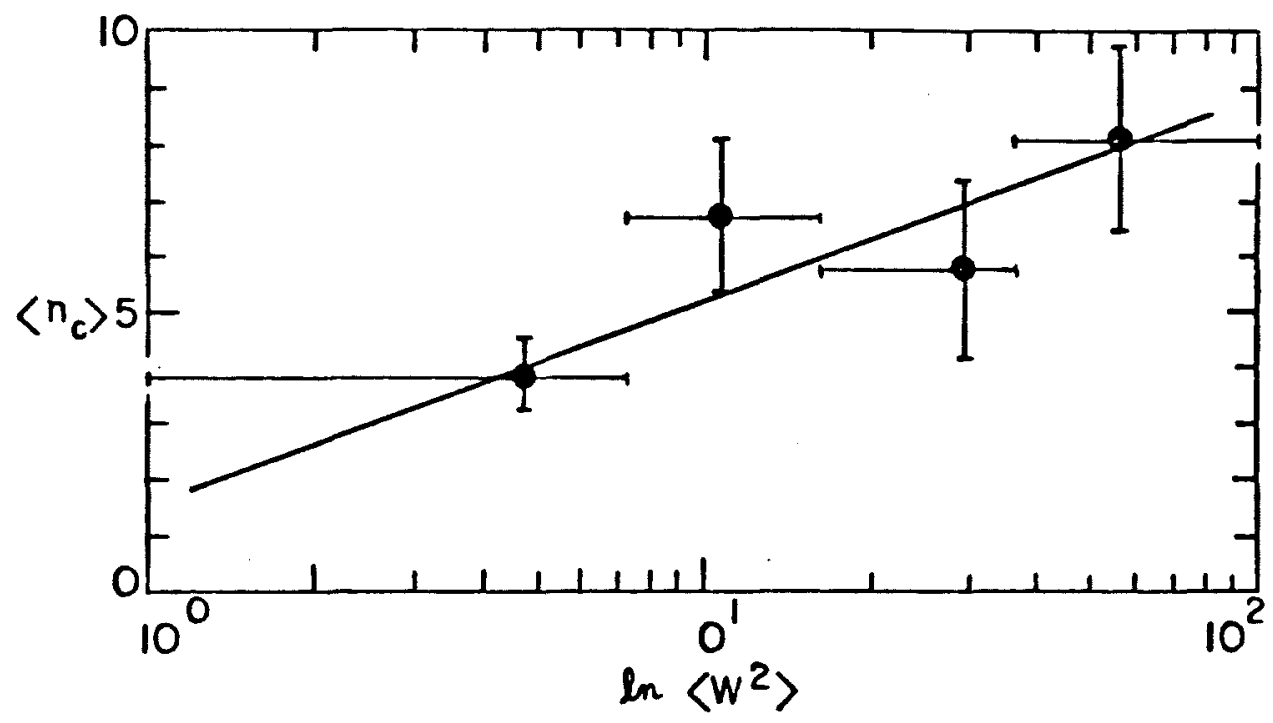


the $W$ dependence quoted above, $X^{2}=0.82 /$ DOF.

To investigate the character of the intranuclear cascade in lepton - nucleus scattering, the normalized multiplicity, $R_{A}$, is of ten used. $R_{A}$ is the ratio of the average number of produced hadrons in lepton - nucleus interactions to the number produced in lepton - nucleon interactions. In figure 3-8 are plotted points for these neutrino - emulsion events $(\langle W\rangle=4.84 \pm 0.52 \mathrm{GeV})$ and neutrino - neon events $(3 \leq W \leq 6 \mathrm{GeV})($ III-2). Also shown are predictions of 3 models: the MCM $(W=4.5 \mathrm{GeV})$, the GLD model (interpolated between $\nu=10$ and $100 \mathrm{GeV}$ to give $\nu=20 \mathrm{GeV}$; the average value of $\nu$ for this experiment is $21.3 \pm 4.4 \mathrm{GeV})$, and the $\operatorname{CTM}(\mathrm{W}=4.8 \mathrm{GeV})$. The MCM curve is well below the results while the GLD and CTM predictions appear to fit the data considerably better.

Wroblewski has shown that the multiplicity dispersion $D^{2}=\left\langle n_{c}^{2}\right\rangle-\left\langle n_{c}\right\rangle^{2}$ rises linearly with the average multiplicity in hadronic interactions (III-14):

$$
D=A<n_{c}>+B
$$

This data sample also exhibits a linear dependence, figure 3-9. The slope parameter found in this experiment is much larger than that found in the neutrino - deuterium interactions and is essentially the same as the hadron - proton result as shown in Table III-1.

Nikolaev has predicted that the ratio $\left(D /\left\langle n_{c}\right\rangle\right)$ is Independent of both the atomic weight of the target and of the number of grey tracks emanating from the event, $\mathrm{N}_{\mathrm{g}}$. These grey tracks have velocities such that $0.3 \leq \beta=v / c \leq 0.7$. For emulsion studies grey tracks are taken to be those tracks with a relative fonization at least 1.4 times the 
54

FIGURE 3-8

Normalized multiplicity, $R_{A}$, versus atomic weight of the target,

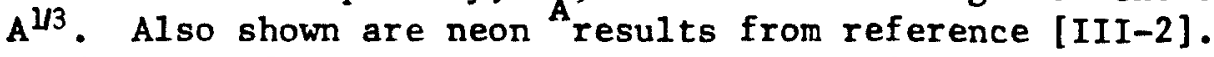

Predictions from the MCM, CTM and GLD models are also displayed. 


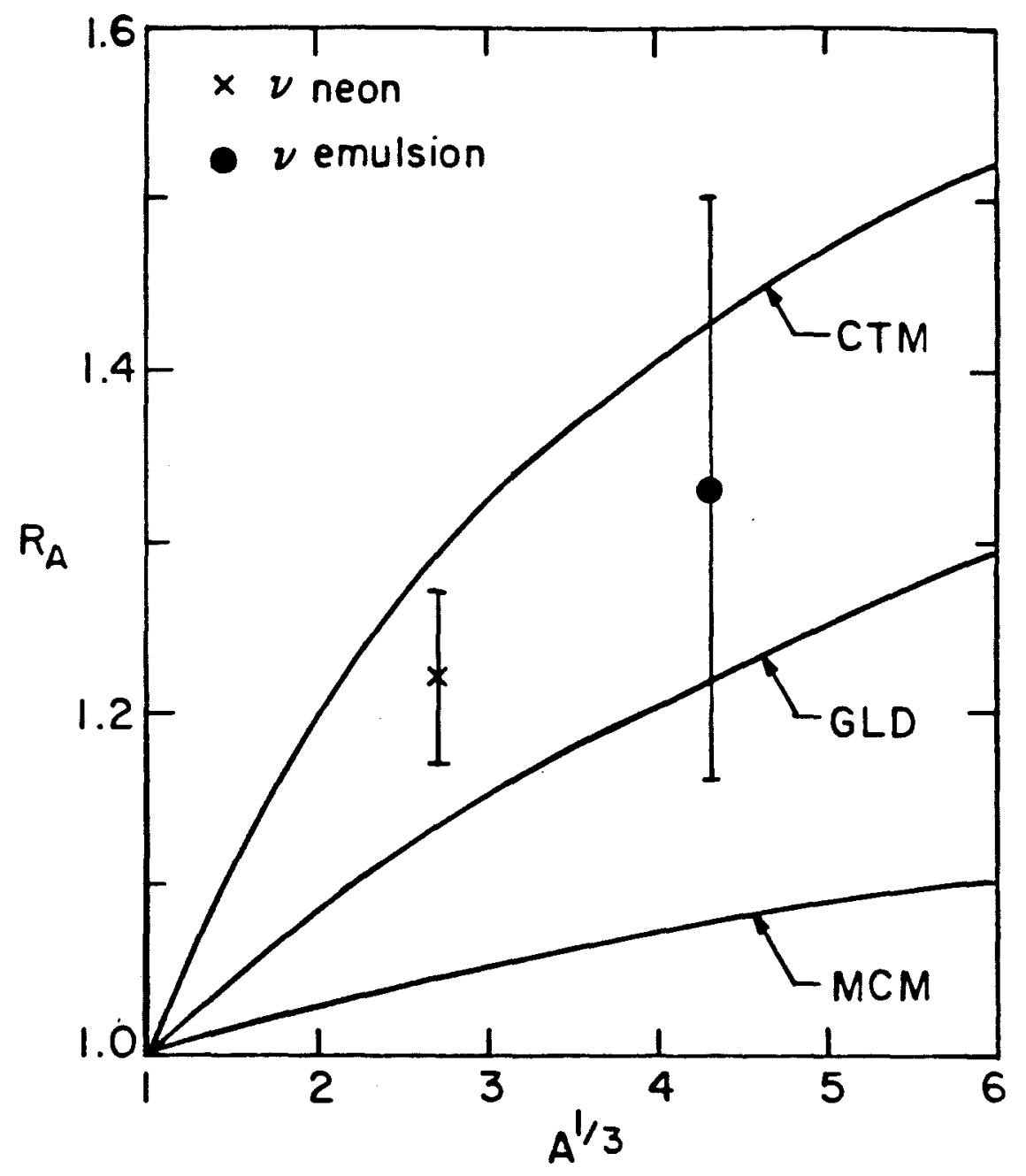


FIGURE 3-9

Dispersion versus the average number of hadrons, $\left(n_{c}\right)$, and linear least squares fit. 


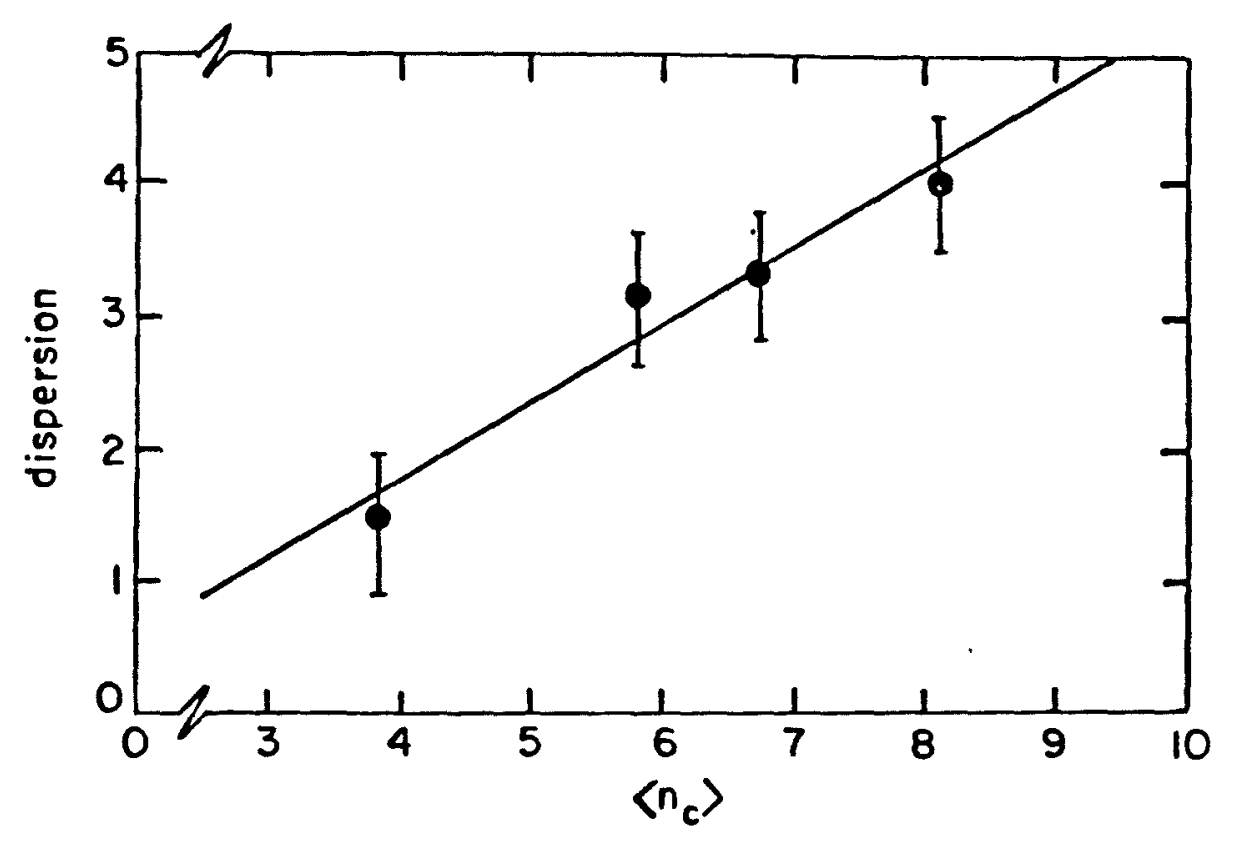


TABLE III-1

Fits to dispersion versus average hadron multiplicity

$$
D=A<n_{c}>+B
$$

\begin{tabular}{lccc}
\hline Interaction & $\mathrm{A}$ & $\mathrm{B}$ & Reference \\
\hline $\mathrm{PP}$ & $0.57 \pm 0.01$ & $-0.54 \pm 0.02$ & III-14 \\
$v-$ Emulsion & $0.59 \pm 0.11$ & $-0.60 \pm 0.67$ & $\begin{array}{l}\text { this exper- } \\
\text { iment } \\
\text { III-9 } \\
v D_{2}-\mathrm{P}\end{array}$ \\
$v \mathrm{D}_{2}-\mathrm{N}$ & $0.34 \pm 0.01$ & $0.16 \pm 0.05$ & III-9 \\
\hline
\end{tabular}


minimum lonization value and a range greater than 3600 microns. Generally these tracks are caused by fast protons. The prediction of $\mathrm{N}_{\mathrm{g}}$ independence is consistent with results of this experiment. The value for low $\mathrm{N}_{\mathrm{g}}$ events $\left(\mathrm{N}_{\mathrm{g}} \leq 2,\left\langle\mathrm{~N}_{\mathrm{g}}\right\rangle=1.26 \pm 0.20\right)$ is $0.53 \pm 0.06$ wh1le that for large $\mathrm{N}_{\mathrm{g}}$ events $\left(\mathrm{N}_{\mathrm{g}} \geq 3,\left\langle\mathrm{~N}_{\mathrm{g}}\right\rangle=4.70 \pm 0.42\right)$ is $0.60 \pm 0.08$. The A dependence, however, differs from the GLD prediction. The value of $D /\left\langle n_{c}\right\rangle$ for this experiment is $0.56 \pm 0.05$, well above the neutrino - proton value of 0.38 (III-12).

Koba, Nielsen and Olesen (KNO) have predicted that asymptotically the multiplicity distribution scaled by the average value of the multiplicity is a function only of the variable $\left.n_{c} /<n_{c}\right\rangle$ (III-15). A result of .KNO scaling is that the ratio $\mathrm{D} /\left\langle\mathrm{n}_{\mathrm{c}}\right\rangle$ is independent of kinematic quantities. Thus the two $\mathrm{N}_{\mathrm{g}}$ samples presented are consistent with obeying kNO scaling with respect to each other. That this ratio differs between proton and emulsion results may show the effect of secondary interactions within the nucleus. It is known that double scattering in deuterfum results in a change in the shape of the multiplicity distribution (III-16). This fact is manifested in the moment, $c_{2}=\left\langle n_{c}^{2}\right\rangle /\left\langle n_{c}\right\rangle^{2}$. Because of the relation:

$$
D /<n_{c}>=\left[\left(c_{2}\right)^{2}-1\right]^{1 / 2}
$$

a change in $c_{2}$ results in a change of $D /<n_{c}>$. The fact that this ratio is apparently independent of $\mathrm{N}_{8}$ but dependent upon $A$ is difficult to reconcile with the correlation between $\mathrm{N}_{8}$ and the number of secondary collisions within the nucleus. More data would be extremely helpful in understanding this question. 
The GLD model yields a prediction for the ratio of the average number of grey tracks, $\left.\mathrm{KN}_{\mathrm{g}}\right\rangle$ for neutrino - emulsion and pion - emulsion interactions which is 0.68 . The experimental comparison with pion events at the same $W$ value is $1.55 \pm 0.25$ (III-17). Thus many more protons are seen to be emanating from neutrino - emulsion collisions than are predicted.

Transverse Momentum Distributions

The transverse momentum distribution with respect to the visible hadronic system for all events is shown in figure 3-10. These results yield an average value of $p_{t}{ }^{2}$ equal to $0.15 \pm 0.02(\mathrm{GeV} / \mathrm{c})^{2}$. This is in agreement with the average value of $\mathrm{p}_{t}{ }^{2}$ from neutrino - neon interactions at the same average $W$ value, $0.153 \pm 0.007(\mathrm{GeV} / \mathrm{c})$ (III-18). The average values of $\mathrm{p}_{t}{ }^{2}$ for events with $\mathrm{N}_{\mathrm{g}} \leq 2$ and $\mathrm{N}_{\mathrm{g}} \geq 3$ are 0.17 $\pm 0.02(\mathrm{GeV} / \mathrm{c})^{2}$ and $0.13 \pm 0.02(\mathrm{GeV} / \mathrm{c})^{2}$ respectively. These values are within experimental errors of each other. The independence of transverse momentum with respect to number of protons has also been found in pion - neon interactions at $W=4.54 \mathrm{GeV}$ (III-19). This has been interpreted to mean that the $\mathrm{p}_{t}{ }^{2}$ distribution is not broadened as more nucleons are involved in the collision process.

Pseudo-Rapidity Distributions

Much detailed information can be gained through examination of the distribution of produced hadrons in rapidity, $y$, and in the frequently used variable pseudo-rapidity, $n$, which closely approximates $y$ when $m_{T} \simeq p_{t}$. The definitions of each variable follow: 
FIGURE 3-10

Distribution of transverse momentum (squared) with respect to the visible hadronic system. 


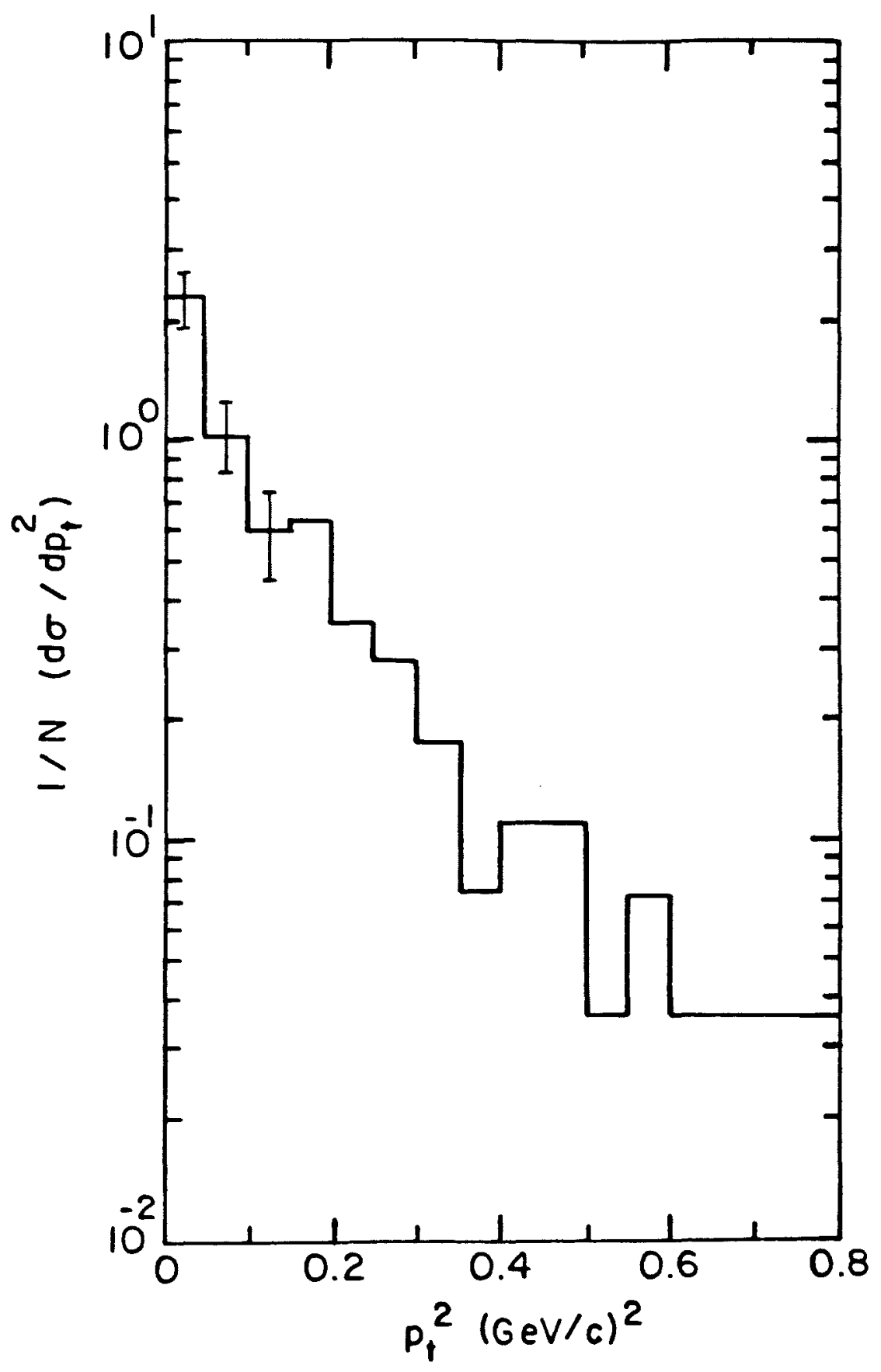




$$
\begin{aligned}
& m_{T}=\left(p_{t}^{2}+m^{2}\right)^{1 / 2} \\
& y=(1 / 2) \ln \left[\left(E+p_{1}\right) /\left(E-p_{1}\right)\right] \\
& n=-\ln [\tan (\theta / 2)]
\end{aligned}
$$

where $E, P_{1}$ and $\theta$ are the energy, longitudinal momentum and the laboratory production angle respectively. Here both $p_{1}$ and $\theta$ are taken with respect to the visible hadronic system. The pseudo-rapidity has been used here because momentum values are avallable for only about $60 \%$ of'the tracks seen in the emulsion.

No difference is observed between the pseudo-rapidity distributions for hadrons from charged current events and from the entire sample, figure 3-11. This indicates similarity of the produced hadronic system whether the incident vector boson is charged or neutral as expected if leading particle effects are ignored. In view of this result subsequent rapidity studies which do not require that the $W$ value be known will use all tracks from the neutral current sample and all hadrons from the charged current sample. Because the neutral current events have an outgoing neutrino with unknown momentum, W cannot be accurately calculated for these events.

To illustrate the level of agreement between $y$ and $n$, Table III-2 gives the ratio of tracks contained in bins of the two variables for a subset of tracks measured in both the emulsion and the bubble chamber. For $66 \%$ of the tracks the fractional difference between the two variables is less than $5 \%$ and for $81 \%$ of the tracks 1 t 18 less than 10\%. Also shown is this ratio derived from measurements of the two quantities for 1250 tracks in an earlier neutrino - neon experiment 
61

FIGURE 3-11

Pseudo-rapidity distributions of hadrons from charged current events. 


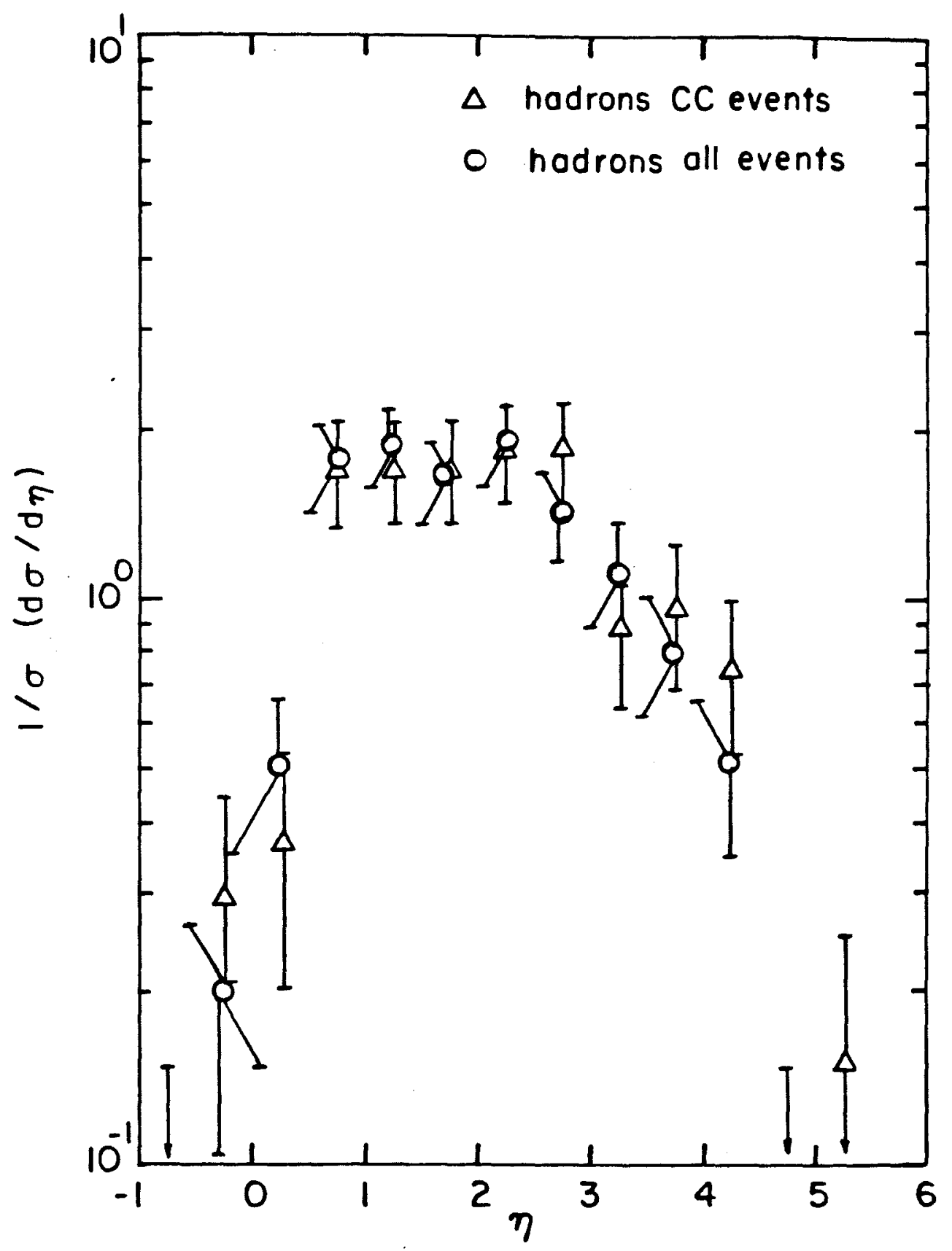


TABLE III-2

Ratios of number of tracks in bins of rapidity to number in bins of pseudo-rapidity - emulsion and neon results [III-20]

\begin{tabular}{lcc}
\hline Range & emulsion ratio $(\# y / \# n)$ & neon ratio $(\# y / \# n)$ \\
\hline$-0.5 \rightarrow 0.5$ & $3.00 \pm 4.73$ & $1.290 \pm 0.218$ \\
$0.5 \rightarrow 1.5$ & $1.14 \pm 0.34$ & $1.221 \pm 0.108$ \\
$1.5 \rightarrow 2.5$ & $1.11 \pm 0.31$ & $1.025 \pm 0.072$ \\
$2.5 \rightarrow 3.5$ & $0.94 \pm 0.33$ & $0.950 \pm 0.074$ \\
$3.5+4.5$ & $0.73 \pm 0.34$ & $0.860 \pm 0.099$ \\
$4.5+5.5$ & $1.25 \pm 1.18$ & $0.708 \pm 0.159$ \\
\hline
\end{tabular}


(III-20). The ratios from the two sources agree with1n errors.

A comparison of rapidity distributions for neutrino interactions with emulsion, neon and hydrogen is made in figure 3-12 (III-2 and III-21). All three data samples have approximately equal values for the hadronic center of mass energy. A correction has been applied to the pseudo-rapidity distribution from emulsion to account for systematic differences between $y$ and $n$. This correction is based on the neon results from Table III-2. Defining the projectile fragmentation region to be the rapidity interval $y \geq 2 \ln \left(W / M_{N}\right)-2$, it can be seen that in this region the emulsion distribution is consistent with but uniformly above the proton and neon results. This is probably due to high $\mathrm{W}$ events in the emulsion sample which have broadened the distribution. As expected, the emulsion distribution is enhanced in the target fragmentation and plateau regions, $y \leq 1$ and $1<y<2 \ln \left(W / M_{N}\right)-2$ respectively, a clear signal for intra-nuclear cascading (III-22).

Assuming a power law dependence:

$$
R_{y}=A^{\alpha}
$$

figure 3-13 gives the value of $\alpha$ as a function of rapidity along with the interpolated prediction from the GLD model. a for the experimental values have been calculated for an effective atomic weight of 80 for emulsion using data from Powell (III-23). The data are seen to lie generally above the prediction. This difference is consistent with the GLD prediction for normalized multiplicity being lower than the data as seen above in figure $3-8$. Pseudo-rapidity distributions for neutrino and muon projectiles 
64

\section{FIGURE 3-12}

Rapidity distributions from neutrino interactions with emulsion, neon [III-2] and protons [III-21]. 


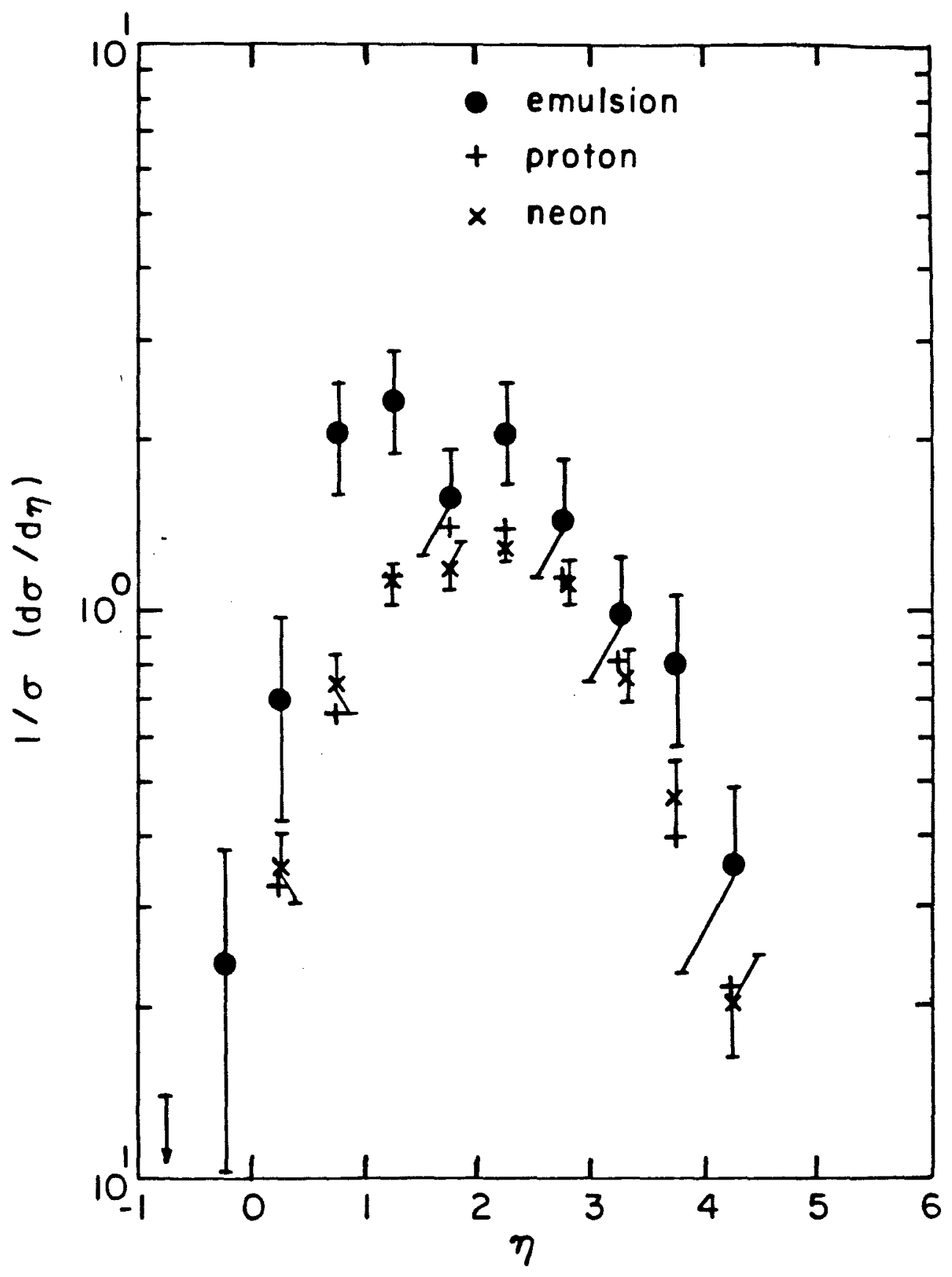


65

FIGURE 3-13

Comparison of emulsion to proton rapidity distributions in neutrino interactions. $\alpha$ versus rapidity (where $R_{y}=A^{\alpha}$ ). 


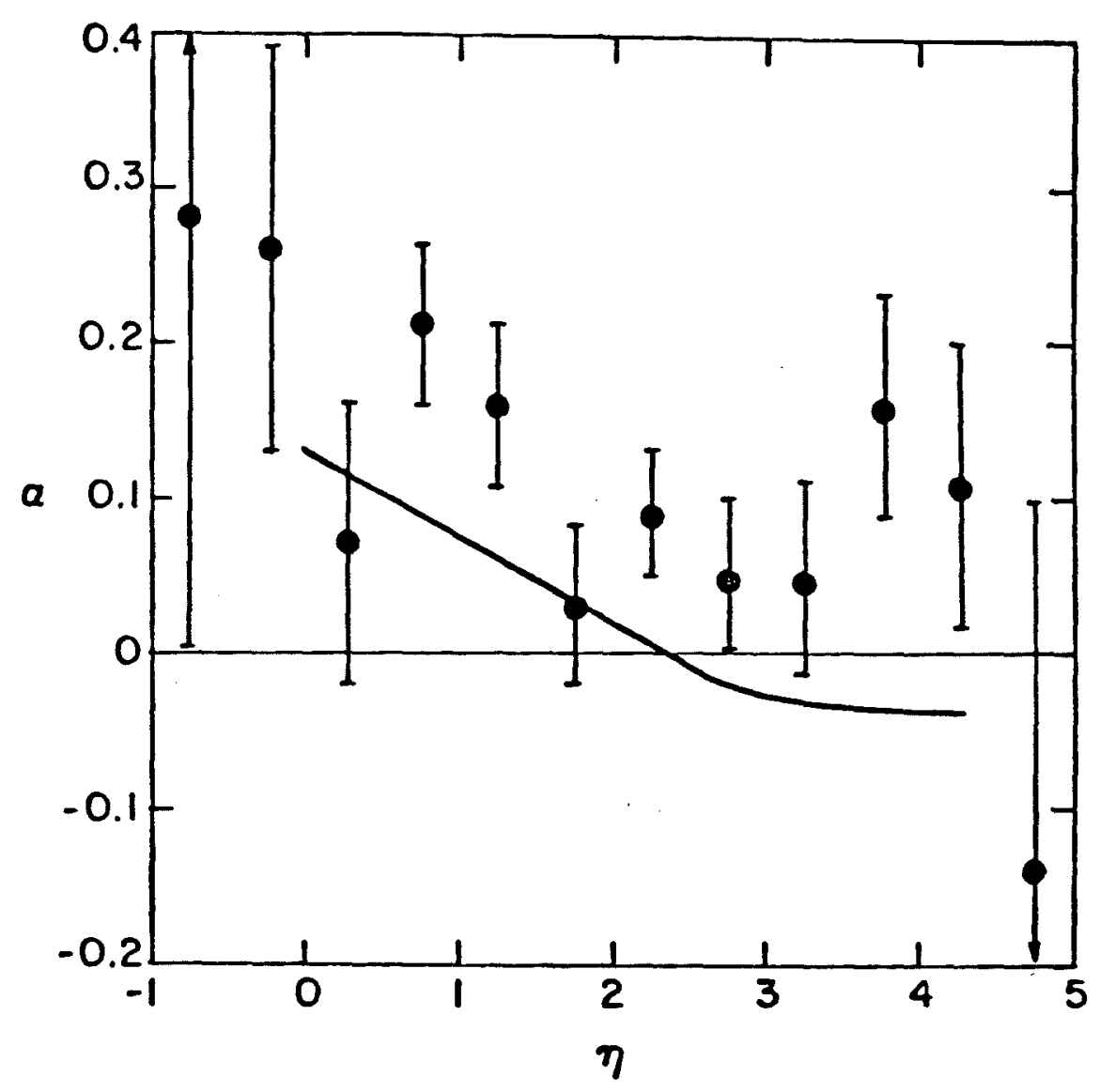


interacting in emulsion are compared in figure 3-14. The muon data is at a mean hadronic center of mass energy of $10.1 \pm 0.4 \mathrm{GeV}$ (III-3). The muon distribution is wider by about $1-1.5$ rapidity units. This is expected as rapidity distributions have a width which grows as approximately $\ln \left(\mathrm{W}^{2}\right)($ III-24).

To test the CTM prediction that

$$
\frac{1}{\sigma} \frac{d \sigma}{d y}\left(W^{2}, y\right)_{A}=\frac{1}{\sigma} \frac{d \sigma}{d y}\left[A^{1 / 3} W^{2}, y+(1 / 3) \ln A\right]_{p}
$$

figure 3-15 displays pseudo-rapidity distributions from this experiment and from $50 \mathrm{GeV} \mathrm{II} \mathrm{-} \mathrm{proton} \mathrm{interactions.} \mathrm{These} \mathrm{two} \mathrm{distributions}$ are at the same CTM effective center of mass energy (III-25). The pion data has been shifted by 1.5 pseudo-rapidity units as prescribed by the CTM. The offset appears to over-correct the difference between proton and emulsion results by about one-half unit of rapidity. Nikolaev (REF Madison) has predicted that a significant dependence on $\mathrm{N}_{g}$, the number of grey tracks, should exist for the pseudo-rapidity distributions in neutrino - emulsion interactions. This prediction assumes that the number of protons measures the occurrence of intranuclear interactions, the source of cascading in the target region. Figure 3-16 shows the ratio between the pseudo-rapidity distributions for 19 events with $\mathrm{N}_{g} \leq 2\left(\left\langle\mathrm{~N}_{\mathrm{g}}\right\rangle=1.26 \pm 0.20\right)$ and for 20 events with $\mathrm{N}_{\mathrm{g}} \geq 3\left(<\mathrm{N}_{\mathrm{g}}>=4.70 \pm 0.42\right)$. Also shown ts the ratio from the GLD prediction for freon $\left(A_{\text {eff }}=50\right)$. The slow change in this ratio with $A$ allows a good comparison between freon and emulsion. A $\chi^{2}$ test between the model and the data yields a confidence level of $75 \%$. However, the $x^{2}$ value for Nikolaev's prediction, $0.55 / \mathrm{DOF}$, is 
67

FIGURE 3-14

Pseudo-rapidity distributions of hadrons from this experiment and from muon-emulsion interactions [III-3]. 


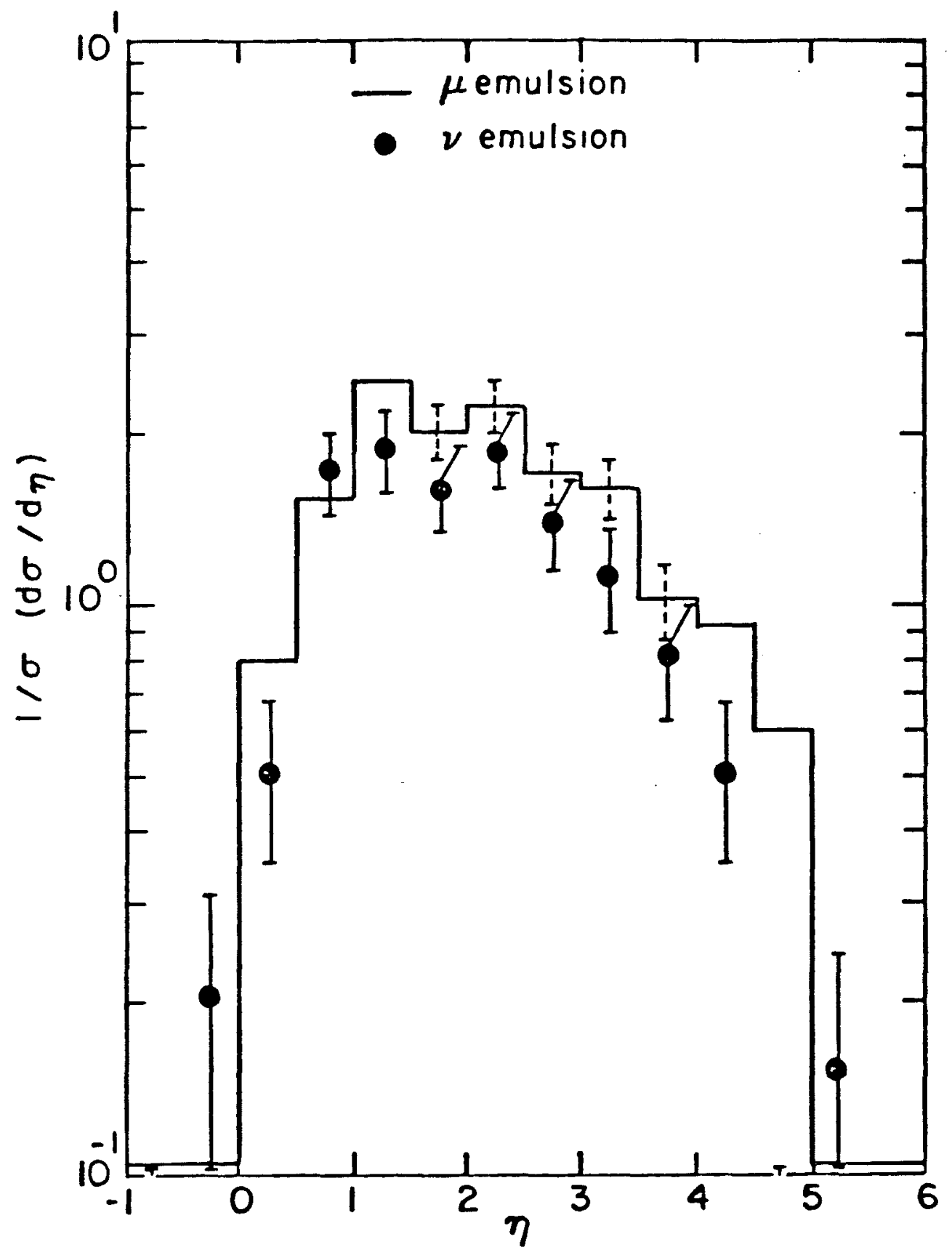


68

FIGURE 3-15

Pseudo-rapidity distributions of hadrons from this experiment and from $\pi$-proton interactions at the same CTM effective energy shifted for A dependence within CTM. 


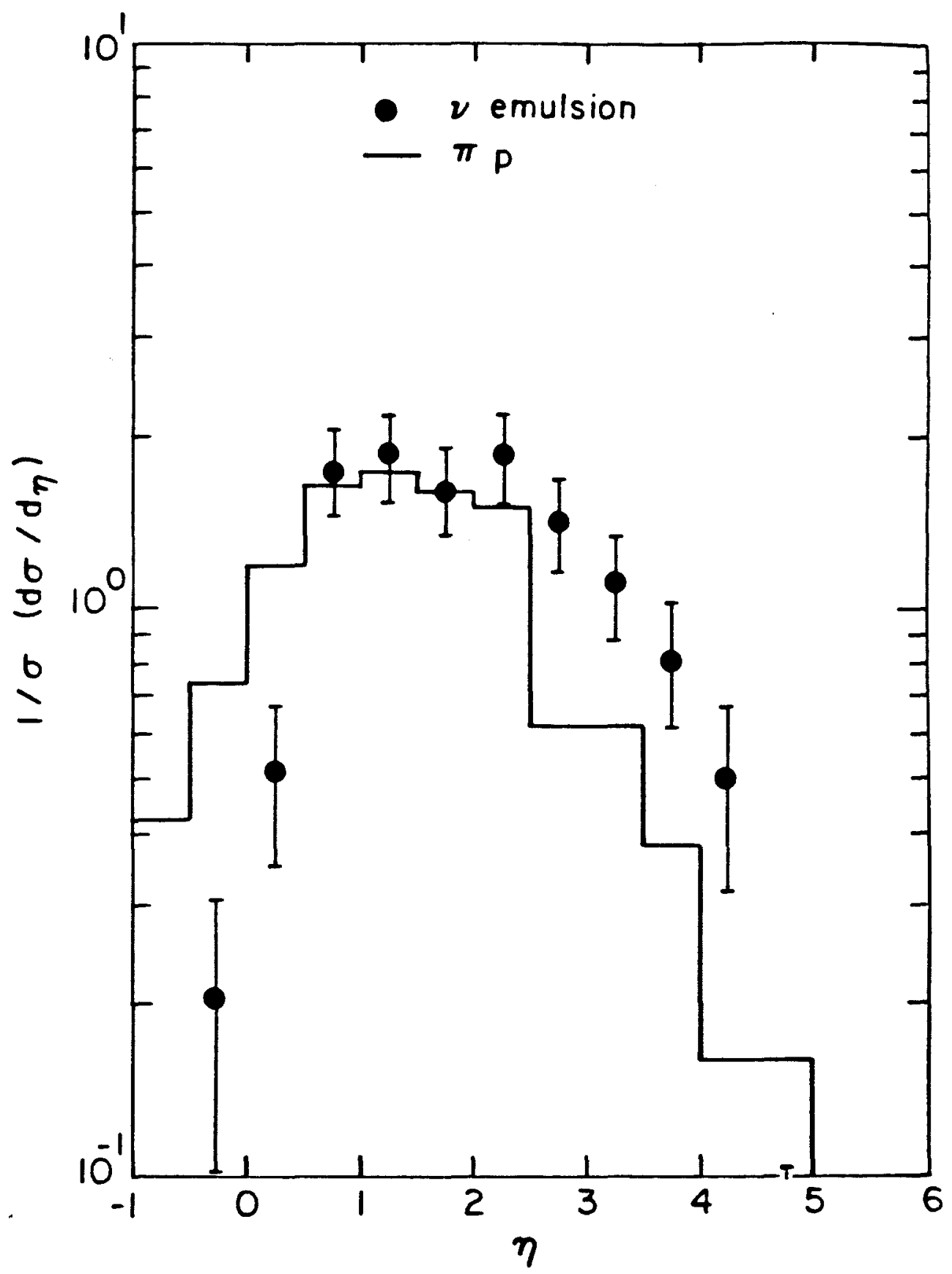


FIGURE 3-16

Ratio of pseudo-rapidity distributions of large and small $\mathrm{N}_{\mathrm{B}}$ data samples versus pseudo-rapidity. Also shown is GLD $B$ prediction for freon at $\nu=10 \mathrm{GeV}$. 


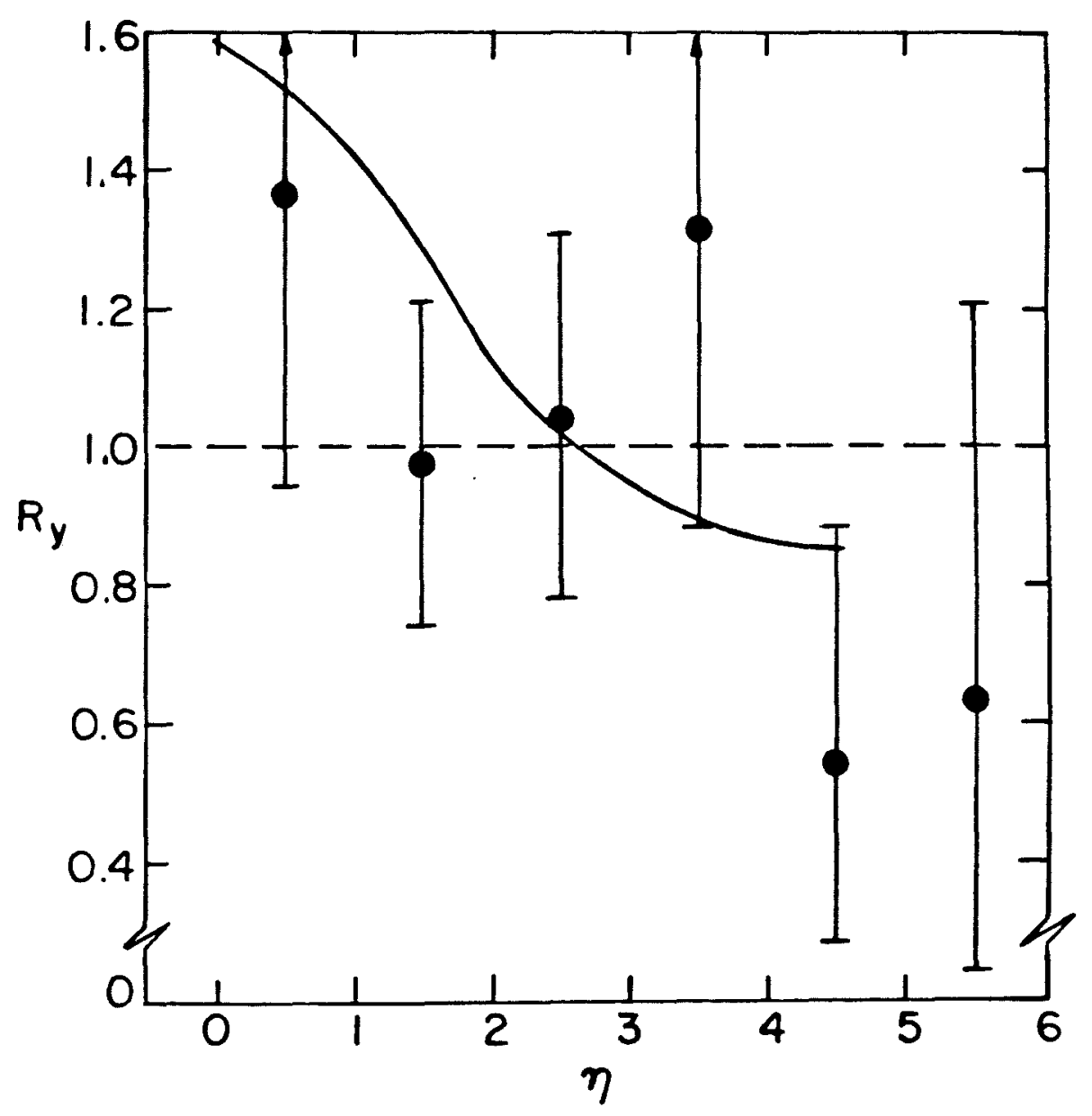


essentially Indistinguishable from that for a constant ratio of unity which yields $x^{2}=0.59 /$ DOF. Therefore, Increased statistics for the data would be very useful for testing the model more fully. 
NOTES TO CHAPTER III

III-1 A. Bialas and W. Czyz, Nuc1. Phys. B137, 359 (1978).

III-2 T. H. Burnett et al., Phys. Lett. $\underline{77 \mathrm{~B}}, 443$ (1978);

T. H. Burnett, Proceedings of the 1977 International

Symposium on Lepton and Photon Interactions at

High Energies, Hamburg (1977) p. 227.

III-3 L. Hand et a1., 2. Phys. C1, 139 (1979).

III-4 M. Hossain and D. Tow, Phys. Rev. D21, 1842 (1980);

G. Bialkowski, C. B. Chiu, and D. M. Tow, Phys. Rev. D17, 862 (1978).

III-5 Y. Afek et al, TECHNION PH-77-83 (1977); Y. Afek et al., Phys. Rev. D15, 2622 (1977).

III-6 N. N. Nikolaev, Z. Phys. C5, 291 (1980); G. V. Davidenko and N. N. Nikolaev, Nucl. Phys. B135, 333 (1978).

III-7 N. N. Nikolaev et al., CERN-TH-2575 (1978); N. N. Nikolaev et al., CERN-TH-2541 (1978).

III-8 J. W. Chapman et al., Phys. Rev. D14, 5 (1976); H. Burmeister and D. C. Cundy, CERN-TC-L Internal Report No. 75-1 (1975).

III-9 J. Hanlon et al., Phys. Rev. Lett. 45, 1817 (1980); T. Kitagaki et al., submitted paper No. 738 to the Twentieth International Conference on High Energy Physics, Madison (1980).

III-10 H. G. Heilmann, CERN WA-21-int-I (1978).

III-11 J. Blietschau et al., Phys. Lett. 87B, 281 (1979).

III-12 N. Schmitz, Proceedings of the 1979 International Symposium on Lepton and Photon Interactions at High Energies, Batavia (1979) P. 359.

III-13 J. Bell et al., Phys. Rev. D19, 1 (1979); J. W. Chapman et al., Phys. Rev. Lett. 36,124 (1976).

III-14 A. Wroblewsk1, Acta Phys. Po1. B4, 857 (1973).

III-15 Z. Koba, H. B. Nielsen, and P. Olesen, Nucl. Phys. B40, 317 (1972).

III-16 H. J. Lubatt1 et al,, Z. Phys. C7, 241 (1981). 
III-17 J. Babecki and G. Nowak, Acta Phys. Pol. B9, 401 (1978).

III-18 H. Deden et al., CERN EP-80-66 (1980).

III-19 W. M. Yeager et al., Phys. Rev. D16, 1294 (1977).

III-20 H. Rudnicka, private communication.

III-21 J. Vander Velde, private communication.

III-22 I. Otterlund, University of Lund, Cosmic and Subatomic Physics Report LUIP 7711 (1977).

III-23 C. F. Powel1, P. H. Fowler, and D. H. Perkins, The Study of Elementary Particles by the Photographic Method, Pergamon Press, New York (1959) P. 42.

III-24 J. D. Bjorken and J. Kogut, Phys. Rev. D8, 1341 (1973); J. D. Bjorken, International Summer Institute on Theoretical Particle Physics, Hamburg (1975) p. 93.

III-25 J. E. Elias et al., Phys. Rev. D22, 13 (1980). 
CHAPTER IV

PRODUCTION AND DECAY OF $\mathrm{F}^{+}$MESON

Theory and Background

In early formulations of a gauge theory for weak interactions, the three original quarks ( $u, d$ and $s$ ) were written in a single left-handed doublet:

$$
\begin{aligned}
& \left(\begin{array}{l}
u \\
d^{\prime}
\end{array}\right) L \\
& \text { where } d^{\prime}=d^{*} \cos \theta_{c}+s^{*} \sin \theta_{c} \\
& \text { and } \theta_{c}=\text { the Cabibbo angle }
\end{aligned}
$$

The form of the weak component of the neutral current predicted by the Weinberg-Salam model is:

$$
\mathrm{J}_{\mathrm{nc}} \sim \sum_{1} \overline{\mathrm{q}}_{\mathrm{i}} \mathrm{T}_{3}(\mathrm{~V}-\mathrm{A}) \mathrm{q}_{i}
$$

where the $q_{i}$ 's are the quark doublet components, $T_{3}$ is the third component of the isospin operator and $(\mathrm{V}-\mathrm{A})$ is the vector minus axial vector space-time structure of the current (IV-1, IV-2).

This predicts that a component of the weak neutral current should be flavor-changing, i.e. mix the d and $s$ quarks with a relative strength proportional to $\sin \theta_{c}{ }^{*} \cos \theta_{c}$. While neutral currents were discovered at CERN in 1973, the flavor-changing neutral currents were not observed (IV-3). An explanation for this is the GIM mechanism (due to Glashow, Illapoulous and Maiani) which proposed the existence of a fourth so-called charmed quark and a second quark doublet:

$$
\left(\begin{array}{l}
c \\
s^{\prime}
\end{array}\right) \text { L }
$$




$$
\text { where } s^{\prime}=8^{*} \cos \theta_{c}-d^{*} \sin \theta_{c}(I V-4) .
$$

With only the first doublet the hadronic neutral current given above yields the form:

$$
\begin{aligned}
J_{n c} & \sim \bar{u} T_{3} u+\bar{d}^{\prime} T_{3} d^{\prime} \\
& \sim \bar{u} u-\bar{d} d \cos ^{2} \theta_{c}-\bar{s} \operatorname{ssin}^{2} \theta_{c}-(\bar{d} s+\bar{s} d) \cos \theta_{c} \sin \theta_{c}
\end{aligned}
$$

Adding in the new doublet, however, gives:

$$
\begin{aligned}
J_{n c} & \sim \bar{u} T_{3} u+\bar{d}^{\prime} T_{3} d^{\prime}+\bar{c} T_{3} c+\bar{s}^{\prime} T_{3} s^{\prime} \\
\sim & \bar{u} u+\bar{c} c-\bar{d} d \cos ^{2} \theta_{c}-\bar{s} \operatorname{ssin}^{2} \theta_{c} \\
& -(\bar{d} s+\bar{s} d) \cos \theta_{c} \sin \theta_{c}-\bar{s} s \cos ^{2} \theta_{c} \\
& -\bar{d} d \sin ^{2} \theta_{c}+(\bar{d} s+\bar{s} d) \cos \theta_{c} \sin \theta_{c}
\end{aligned}
$$

$\sim \bar{u} u+\bar{c} c-\bar{d} d-\bar{s} s$

This results in the cancellation of all non-diagonal terms in the weak neutral current at the expense of one new quark.

Following the simultaneous discovery of the $\mathrm{J} / \psi$ particle at SLAC and Brookhaven, the new particle was quickly interpreted as a cic pair; that is, a particle which is a manifestation of charm but which has a net value of zero for the charm quantum number (IV-5).

New weakly decaying mesons, D's, were observed at the SPEAR and DESY $e^{+} e^{-}$colliding beam machines and new baryons, $\Lambda_{c}^{+}$'s were seen in a photoproduction experiment (IV-6, IV-7). These particles which explicitly carry the charm quantum number had been detected through their decay products, allowing measurement of branching ratios and masses. But final confirmation of the existence of the charmed quark awaited the direct measurement of their decay times. 
A simple prediction for the semileptonic decay rate of the charmed particle can be made by assuming that the other constituents of the particle act only in a spectator role. Then the calculation reduces to scaling the Fermi calculation of the muon decay rate. Figure 4-1 A and B show the appropriate Feynman diagrams.

$$
\begin{aligned}
& \Gamma\left(c \rightarrow s 1^{+} \nu_{1}\right)=\Gamma\left(\mu^{+}+e^{+} \nu_{e} \bar{\nu}_{\mu}\right)\left(\frac{m_{c}}{m_{\mu}}\right)^{5} \cos ^{4} \theta_{c} \\
& \simeq 3 \times 10^{11} s^{-1} \\
& \text { using }\left(m_{J / \psi}\right) / 2 \text { for } m_{c} \text {. }
\end{aligned}
$$

Because the $\mathrm{W}^{+}$can decay into 2 types of lepton pairs and 3 colors of quark pairs, a naive estimate of the semi-leptonic branching ratio is $1 / 5$. Because the mass of the tau lepton is so close to that of the lowest mass charmed particles, it has been eliminated from consideration here. This simple model gives a charm particle lifetime of about $7 \times 10^{-13}$ second independent of the specific parent particle.

This model can be applied to the decay of strange particles although the smaller strange quark mass weakens the spectator assumption for the other quarks. Specifically the $K_{L}^{0}$ has an analogous three body semi-leptonic decay mode. For example, using a value of $30 \%$ for the $\mathrm{K}_{\mathrm{L}}^{0}$ semi-leptonic branching ratio and $300 \mathrm{MeV} / \mathrm{c}^{2}$ as the strange quark mass gives the following results which can be compared with the measured $\mathrm{K}_{\mathrm{L}}^{0}$ lifetime of $5 \times 10^{-8}$ seconds.

$$
\begin{aligned}
\Gamma\left(s+u 1-\bar{v}_{1}\right) & =\Gamma\left(\mu^{+}+e^{+} v_{e} \bar{v}_{\mu}\right)\left(\frac{m_{s}}{m_{\mu}}\right)^{5} \cos ^{2} \theta_{c} \sin ^{2} \theta_{c} \\
& \simeq 4 \times 10^{6} s-1 \\
\tau & =B R / \Gamma \simeq 7 \times 10^{-8} s
\end{aligned}
$$


FIGURE 4-1

Feynman diagrams of: 4-1a muon decay, 4-1b semi-leptonic decay of $D$ or $F$ in spectator model, 4-1c W-exchange in $D^{\circ}$ decay, anniliation in F decay. 

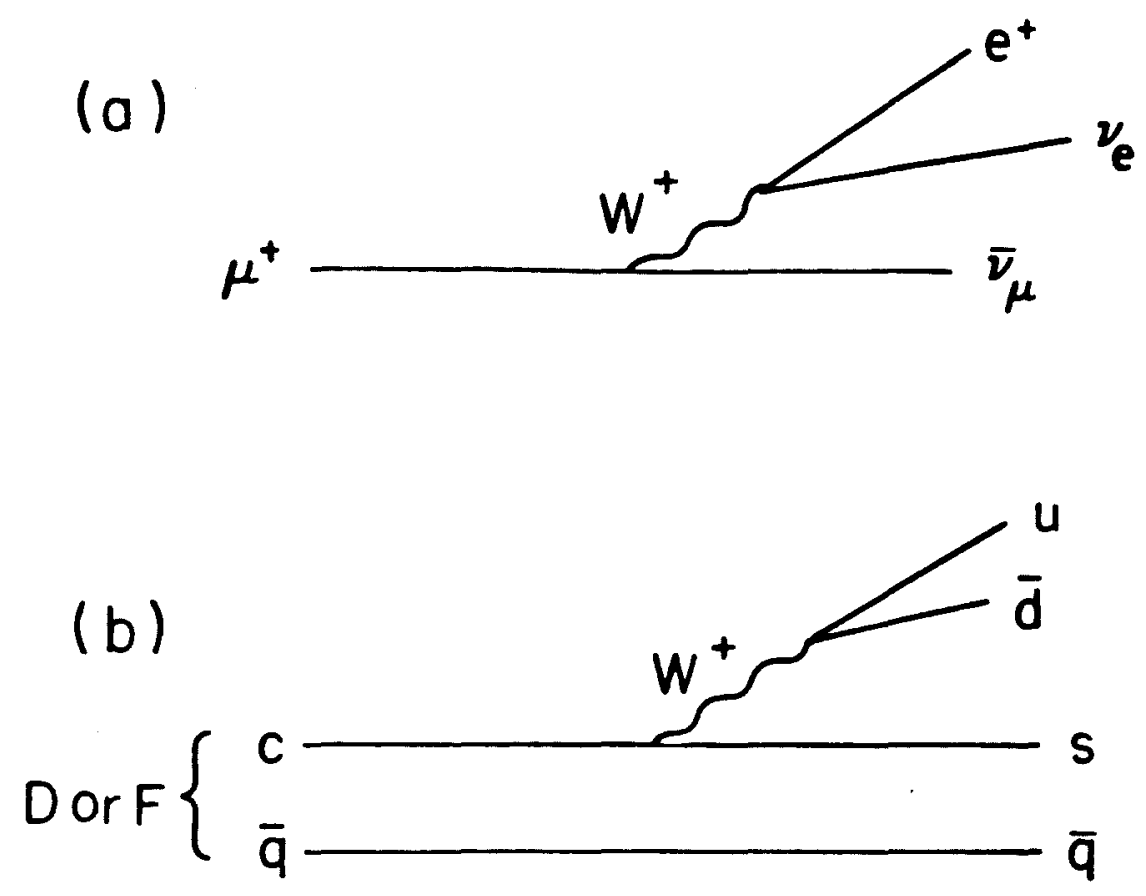

(c)

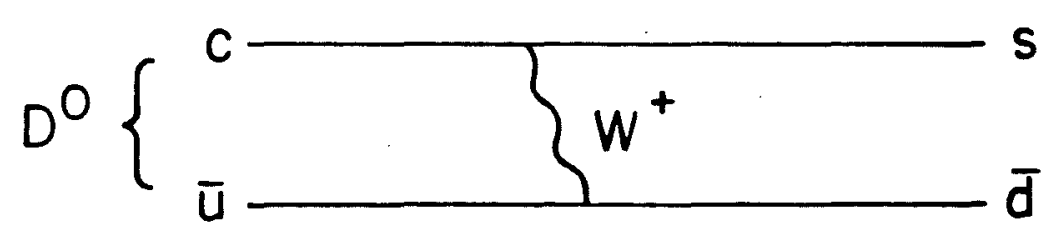

(d)

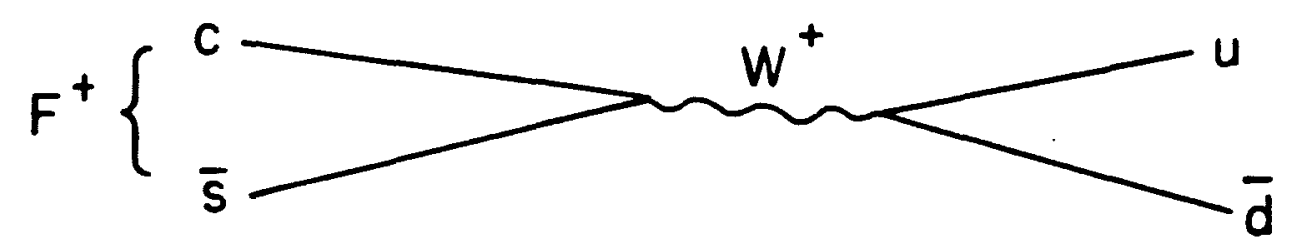


The first charm decays observed in emulsion did not have external spectrometers and gave limited information on the parent particles, but Indicated a charm Iffetime of about $10^{-13}$ seconds (IV-8). Early hybrid emulsion experiments have detected the decays of a small sample of charmed particles, some of which have been Identified as $D^{0}, D^{ \pm}$and $\Lambda_{c}^{+}$, and confirmed that the lifetimes are approximately those predicted by the spectator model (IV-9).

A more recent hybrid-emulsion experiment, Fermilab experiment E531, has observed 29 decays of charmed particles and published results which show that the lifetime of the $D^{ \pm}$is significantly longer than that of the $D^{0}:($ IV-10)

$$
\begin{aligned}
& \tau_{D^{ \pm}}=10.3_{-4.1}^{+10.5} \times 10^{-13} \mathrm{~s} \\
& \tau_{D} 0=1.01_{-0.27}^{+0.43} \times 10^{-13} \mathrm{~s}
\end{aligned}
$$

This result is consistent with the ratios of semileptonic branching ratios observed in decays at $\mathrm{e}^{+} \mathrm{e}^{-}$machines: (IV-11)

$$
\begin{aligned}
\Gamma\left(D^{0}\right) / \Gamma\left(D^{ \pm}\right) & =3.1_{-1.4}^{+4.2} & \text { MARK II } \\
& >4.3 \text { (95\% C.L.) } & \text { DELCO }
\end{aligned}
$$

Several authors have presented explanations of this difference in lifetimes (IV-12). If diagrams involving W-exchange or quark annihilation into $a \mathrm{~W}$ are important, figure 4-1 C and $D$, then the $\mathrm{D}^{0}$ and $\mathrm{F}^{+}$decay rates respectively would be enhanced. Early est1mates of charm decay rates ignored contributions from diagrams such as these because of helicity suppression. The initial meson, $D^{0}$ or $\mathrm{F}^{+}$, Is a pseudo-scalar state. Because of its $(\mathrm{V}-\mathrm{A})$ nature, the weak 
Interaction couples to left-handed quarks and right-handed antiquarks. In the limit of zero masses for the final state quarts in figure 4-1 C and D, the quark (antiquark) would necessarily have its spin anti-aligned (aligned) with its momentum. In the rest frame of the parent particle the decay products have equal and opposite momentum vectors and spins which are in the same direction, 1.e. $\mathrm{J}=1$. Therefore, both linear and angular momenta can not be simultaneously conserved.

Because the final state quarks ( $u$, $d$ or $s$ ) have non-zero masses, their helicities are not absolutely determined. Although the above suppression is not total, the decay rate to a $\mathrm{J}=0$ state is reduced by a factor of $\left(m_{q} / m_{p s}\right)^{2}$ which is equal to a few percent. Here, $m_{q}$ is the light quark mass and $m_{p s}$ is the mass of the pseudoscalar meson.

A possible mechanism to circumvent this suppression is gluon emission from the initial state. Since a gluon has spin 1 , the qa system can have $J=1$ while the total angular momentum of the $q \bar{q}+$ glue system is zero as it must be. The emission of a single gluon (color octet) leaves the $c \bar{q}$ of the charmed meson in a color octet state which will not couple to the color singlet $W$ boson. Thus soft gluon processes are probably necessary to carry off this color, allowing a color singlet $c \bar{q}$ state.

Estimates by Shizuya and Fritsch - Minkowski are that the relative lifetimes of the charmed pseudoscalar mesons are:

$$
\tau\left(D^{0}\right) \lesssim \tau\left(F^{+}\right)<\tau\left(D^{ \pm}\right)
$$

specifically, Shizuya estimates: 


$$
\frac{\Gamma_{8 g}\left(F^{+}\right)}{\Gamma_{8 g}\left(D^{0}\right)}=0.4
$$

where $\Gamma_{8 g}$ is the rate for the soft gluon process. W1th the observed D lifetimes quoted above this yields the value of $2.4 \times 10^{-13}$ seconds for the $\mathrm{F}^{+}$iifetime.

If these predictions are correct, then there should be a significant fraction of $F$ decays without a strangeness content. If the $F$ decays only through $W$-radiation, then the final state should contain both an $s$ and an $\bar{s}$ quark.

\section{Experimental Results}

In the tagged neutrino event sample an example has been found of

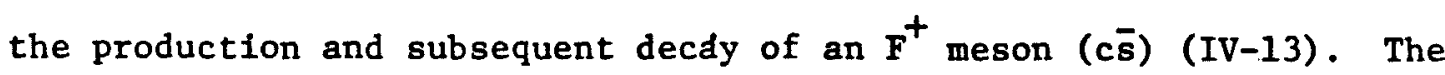
event is shown in Plate 4 as it appears both in the bubble chamber and in a camera lucida drawing from the emulsion. Four minimum ionizing tracks and two heavily lonizing nuclear fragments with ranges of less than 500 microns emanate from the neutrino vertex (A). Track parameters as measured in the emulsion and from the bubble chamber film are listed in Table IV-1. The bubble chamber angles have been swum back to the vertex and converted to the emulsion co-ordinate system.

Track 1 is identified as a muon by hits in both planes of the EMI. There is a small 11kelihood of track 3 being caused by a particle more massive than a pion due to the combination of low momentum and minimum ionization. Three tracks (5, 6 and 7) visible in the bubble chamber are not seen in the emulsion and are consistent with being 
80

PLATE IV

Neutrino event with $\mathrm{F}^{+}$decay as seen in emulsion and bubble chamber. 

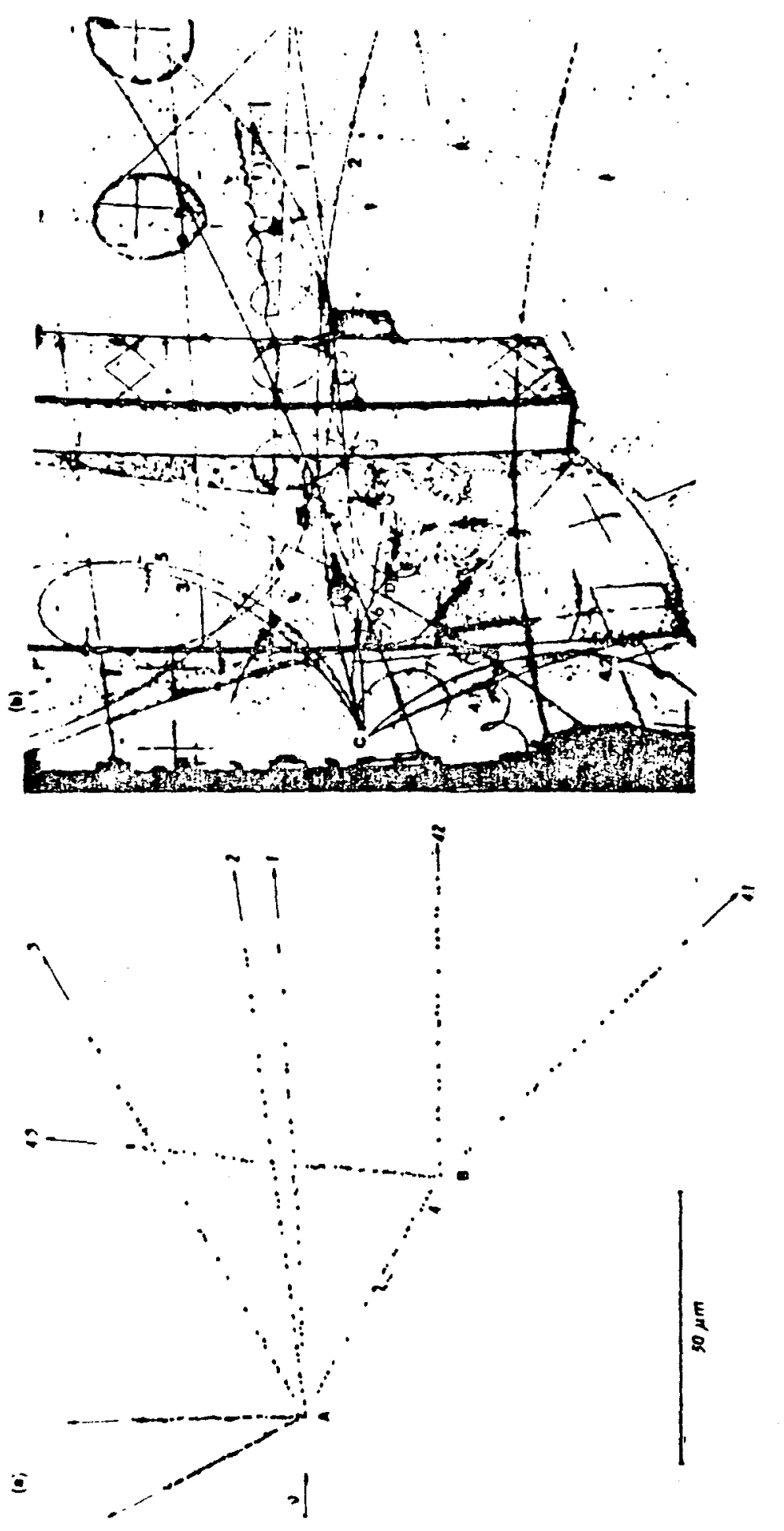
TABLE IV-1

Measured track parameters for event

\begin{tabular}{|c|c|c|c|c|c|c|}
\hline Track & $\begin{array}{l}\text { Emulsion data } \\
\text { Azimuth } \\
\text { (deg.) }\end{array}$ & $\begin{array}{l}\text { D1p } \\
\text { (deg.) }\end{array}$ & $\begin{array}{l}\text { Bubble Cham } \\
\text { Azlmuth } \\
\text { (deg.) }\end{array}$ & $\begin{array}{l}\operatorname{ata}^{a} \\
\quad \text { Dip } \\
\text { (deg.) }\end{array}$ & $\begin{array}{l}\text { Momentum } \\
(\mathrm{GeV} / \mathrm{c})\end{array}$ & Charge \\
\hline $\begin{array}{l}v \\
1 \\
2 \\
3 \\
4^{b} \\
4.1 \\
4.2 \\
4.3 \\
5 \\
6 \\
7\end{array}$ & $\begin{aligned}-3.0 & \pm 0.5 \\
2.8 & \pm 0.1 \\
8.4 & \pm 0.1 \\
30.4 & \pm 0.1 \\
-28.8 & \pm 0.2 \\
-45.1 & \pm 0.2 \\
-2.3 & \pm 0.3 \\
86.4 & \pm 0.4\end{aligned}$ & $\begin{aligned}-2.5 & \pm 0.5 \\
-5.0 & \pm 0.3 \\
-18.7 & \pm 0.5 \\
-28.4 & \pm 0.5 \\
4.4 & \pm 0.7 \\
11.7 & \pm 0.4 \\
41.3 & \pm 0.3 \\
-62.4 & \pm 0.4\end{aligned}$ & $\begin{aligned} 2.3 & \pm 0.5 \\
8.2 & \pm 0.7 \\
28.7 & \pm 2.8 \\
-44.3 & \pm 0.6 \\
-1.3 & \pm 3.2 \\
33.4 & \pm 1.9 \\
32.7 & \pm 3.0 \\
3.9 & \pm 6.9\end{aligned}$ & $\begin{aligned}-5.5 & \pm 0.5 \\
-18.3 & \pm 0.7 \\
-26.1 & \pm 2.4 \\
& \\
10.4 & \pm 0.6 \\
38.8 & \pm 2.5 \\
-20.3 & \pm 1.7 \\
-20.3 & \pm 2.6 \\
-37.4 & \pm 4.7\end{aligned}$ & $\begin{aligned} 12.02 \pm 0.11 \\
1.37 \pm 0.01 \\
0.314 \pm 0.005 \\
\\
2.01 \pm 0.01 \\
0.299 \pm 0.003 \\
B=0.65 \pm 0.06^{C} \\
0.257 \pm 0.007 \\
0.160 \pm 0.007 \\
0.049 \pm 0.020\end{aligned}$ & $\begin{array}{l}- \\
+ \\
- \\
+ \\
+ \\
+ \\
+ \\
+\end{array}$ \\
\hline
\end{tabular}

a Tracks have all been swum back to their respective origins and their angles transformed to the same reference frame as the emulsion data.

b This track decays after traveling $50 \pm 1 \mu \mathrm{m}$.

c This track does not enter the bubble chamber. The value for $\beta$ is obtalned from lonization measurements in the emulsion. 
the conversion products of two photons. Both the difference in angles and the spatial separation of tracks 5 and 6 are a minimum at a point 3.2 into the stainless steel of the box which 1s thus taken as the conversion point of $\gamma_{1}$. Track 7 is assumed to be from a photon conversion $\left(\gamma_{2}\right)$ from which the electron is not observed. The conversion point of this photon has been determined by tracing track 7 upstream until a minimum difference between its track angles and the direction to the decay vertex has been found.

After a flight length of 50 microns, track 4 decays into three charged particles at vertex B. One of the daughter particles (4.3) does not reach the bubble chamber, so its velocity has been determined by ionization measurements in the emulsion. Its charge has been determined to be negative because both of the other two decay products are positive. Another track (4.1) has a transverse momentum of $610 \mathrm{MeV} / \mathrm{c}$ with respect to the direction of the parent. The summed transverse momentum of the charged decay products with respect to the parent direction is $0.53 \pm 0.02 \mathrm{GeV} / \mathrm{c}$ which $1 \mathrm{mplies}$ the existence of one or more neutral particles emerging from the decay vertex.

The three charged plus neutral(s) decay topology eliminates from consideration as possible background known strange particle decay modes which could pose as charm decay. The probability of a secondary hadronic interaction with this form can be calculated to be $1 \times 10^{-3}$ using a 12 meter interaction length for hadrons in emulsion leading to an event with no nuclear fragments $\left(\mathrm{N}_{h}=0\right)$ and having an odd number of produced charged tracks (IV-14).

Using the three charged particles, 0-C kinematic fits were 
attempted for both charm and non-charm decays. The only fits thus obtained were to the $\mathrm{D}^{+}, \mathrm{F}^{+}$and $\Lambda_{\mathrm{c}}^{+}$, all with at least one missing neutral.

Acknowledging the presence of a neutral (1.e. $\pi^{0}+\gamma_{1} \gamma_{2}$ or just $\gamma_{1}$ ) four 3-C fits were obtained, three to the $\mathrm{F}^{+}$and one to the $\Lambda_{c}^{+}$ (see Table IV-2). The lambda decay is removed from consideration by the combination of its large chi-squared value and Its Cablbbo suppression.

The selection of the $\mathrm{F}^{+}(2030)+4 \pi$ mode on the basis of 1 ts high probability leads to the fitted track parameters shown in Table IV-3. The invariant mass of the $\left(4.2,4.3, \pi^{0}\right)$ system is $808 \pm 20$ $\mathrm{MeV}$, near the $\omega$ mass and inconsistent with either the $n$ or $\phi$. The $\left(4.1,4.3, \pi^{0}\right)$ mass is near $1800 \mathrm{MeV}$.

The proper time for the decay is $1.4 \times 10^{-13}$ seconds. Treating the parent mass as unknown gives a $2-C$ fit and a value for the $\mathrm{F}^{+}$ mass assignment of $2017 \pm 25 \mathrm{MeV} / \mathrm{c}^{2}$.

For this event the visible energy is $16.2 \mathrm{GeV}$ and the corrected energy is $18.5 \mathrm{GeV}$. The uncorrected $x$ and $y$ values are $0.30 \pm 0.05$ and $0.26 \pm 0.01$ respectively. These values are well within the phase space limits for charm production given by: (figure 4-2)

$$
\begin{aligned}
& 0 \leq x \leq 1-\frac{E_{t h}}{y E_{\nu}} \\
& \frac{E_{t h}}{E_{\nu}} \leq y \leq 1
\end{aligned}
$$

where $E_{t h}=\left(m_{c}^{2}-m_{p}^{2}\right) / 2 m_{p}$ is the threshold energy for charm production (IV-15). 
TABLE IV-2

3-C fits obtained

\begin{tabular}{lll}
\hline Hypothesis & $\mathrm{x}^{2}$ & Probability \\
\hline $\mathrm{F}^{+}(2030)+\pi^{+} \pi^{+} \pi^{-} \pi^{0} \gamma_{1} \gamma_{2}$ & 3.77 & $29 \%$ \\
$\mathrm{~K}^{+} \pi^{+} \pi^{-} \pi^{0}$ & 12.7 & $0.5 \%$ \\
$\pi^{+} \pi^{+} \pi^{-} \gamma_{1}$ & 16.7 & $0.08 \%$ \\
$\Lambda_{\mathrm{C}}^{+}(2257)+\mathrm{p}^{+} \pi^{-} r^{0} \gamma_{2}$ & & \\
\hline
\end{tabular}


TABLE IV-3

Fit results for $\mathrm{F}^{+}(2030) \rightarrow \pi^{+} \pi^{+} \pi^{-} \pi^{0}$, with $\pi^{0} \rightarrow \gamma_{1} \gamma_{2}$

\begin{tabular}{llrrl}
\hline Track & Identity & \multicolumn{1}{c}{$\begin{array}{l}\text { Azimuth } \\
\text { (deg.) }\end{array}$} & \multicolumn{1}{l}{$\begin{array}{l}\text { Dip } \\
\text { (deg.) }\end{array}$} & $\begin{array}{l}\text { Momentum } \\
\text { (GeV/c) }\end{array}$ \\
\hline 4 & $\mathrm{~F}_{+}^{+}(2030)$ & $-28.7 \pm 0.2$ & $5.1 \pm 0.4$ & $2.37 \pm 0.03$ \\
4.1 & $\pi^{+}$ & $-45.2 \pm 0.2$ & $11.5 \pm 0.4$ & $2.01 \pm 0.01$ \\
4.2 & $\pi^{-}$ & $-2.3 \pm 0.3$ & $41.3 \pm 0.3$ & $0.299 \pm 0.003$ \\
4.3 & $\pi^{0}$ & $86.4 \pm 0.4$ & $-62.4 \pm 0.4$ & $0.133 \pm 0.018$ \\
& $\pi^{0}$ & $25.0 \pm 2.2$ & $-28.2 \pm 1.6$ & $0.568 \pm 0.028$ \\
& $\gamma_{1}$ & $32.1 \pm 1.9$ & $-22.8 \pm 1.6$ & $0.415 \pm 0.010$ \\
& $\gamma_{2}$ & $3.5 \pm 3.4$ & $-39.7 \pm 3.9$ & $0.169 \pm 0.029$ \\
\hline
\end{tabular}


86

FIGURE 4-2

Phase space limits for charm production in $\mathrm{F}^{+}$event and location of the event in phase space. 


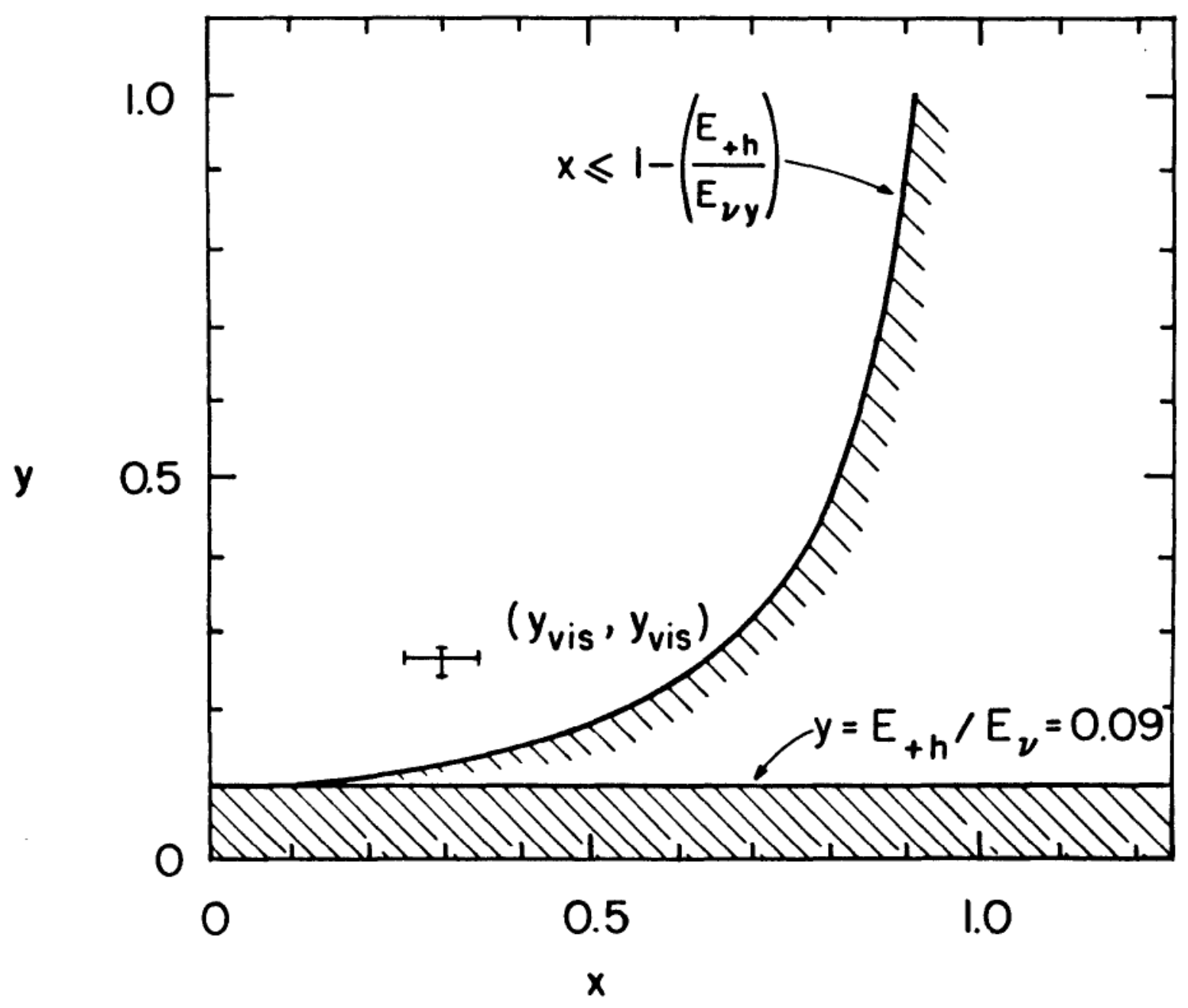


The $\mathrm{F}^{+}$was first detected at DORIS by the DASP collaboration through the $n \pi$ mode (IV-16). The reported mass value $182030 \pm 60$ $\mathrm{MeV} / \mathrm{c}^{2}$. The only directly detected F's are from this experiment and E5 31 which has seen two decays. Table IV-4 summarizes the $\mathrm{F}^{+}$decays seen in emulsion. A likelihood function can be formed from this sample:

$$
\mathcal{L}(\tau)=\prod_{1}\left[(1 / \tau) \exp \left(-t_{1} / \tau\right)\right]
$$

which yields a lifetime of $2.01(+1.80,-0.82) \times 10^{-13}$ seconds which is very close to the estimate based on Shizuya's analysis. Figure 4-3. Because the $\mathrm{F}^{+}$Iifetime is close to that of the $D^{0}$ and because only one of the three events has a two kaon final state, this small data sample appears to argue against W-radiation being the dominant decay mode for this particle. The fact that two of the decays are to four pion states is very suggestive that the $c \bar{s}$ annihilation diagram is significant and hence that gluon emission is decreasing the effect of the helicity suppression. 
TABLE IV -4

Summary of $\mathrm{F}^{+}$decays seen in emulsion

\begin{tabular}{llllll}
\hline Experiment & Decay & Decay Length & F Momentum & F Mass & Decay Time \\
\hline E564 & $\mathrm{F}^{+} \rightarrow \pi^{+} \pi^{+} \pi^{-} \pi^{0}$ & $50 \mathrm{~m}$ fcrons & $2.37 \mathrm{GeV} / \mathrm{c}$ & $2017 \pm 25\left(\mathrm{MeV} / \mathrm{c}^{2}\right)$ & $1.4 \times 10^{-13} \mathrm{~s}$ \\
E531 & $\mathrm{F}^{-}+\pi^{-} \pi^{-} \pi^{+} \pi^{0}$ & 670 & 12.25 & $2026 \pm 56$ & 3.7 \\
E531 & $\mathrm{F}^{+} \rightarrow \mathrm{K}^{+} \pi^{-} \pi^{+} \mathrm{K}^{0}$ & 130 & 9.70 & $2089 \pm 121$ & 0.9 \\
\hline
\end{tabular}


89

FIGURE 4-3

Likelihood function for $\mathrm{F}^{+}$lifetime calculated from results of this experiment and reference [IV-10]. 


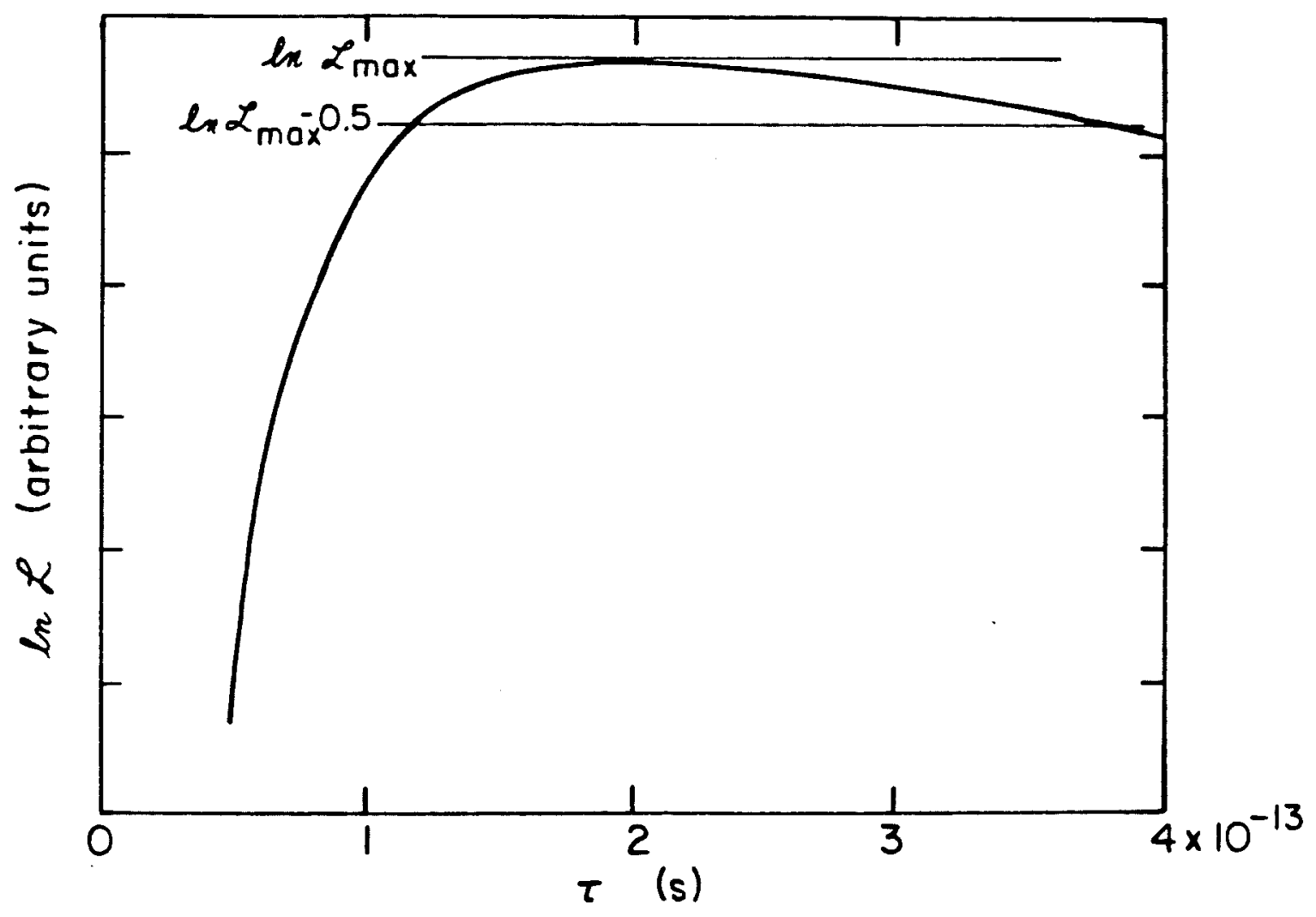


NOTES TO CHAPTER IV

IV-1 A. Salam, J. C. Ward, Phys. Lett. 13, 168 (1964); S. Welnberg, Phys. Rev. Lett. 19, 1264 (1967); S. Welnberg, Phys. Rev. D5, 1412 (1972).

IV-2 A second term in the weak neutral current is diagonal and has been ignored here. C. Quigg, FNAL PUB CONF 78/79 - THY (1978).

IV-3 F. Hasert et al., Phys. Lett. 46B, 121 and 138 (1973).

IV-4 S. Glashow, J. Illapoulous, L. Malan1, Phys. Rev. D2, 1285 (1970).

IV-5 J. J. Aubert et al., Phys. Rev. Lett. 33, 1404 (1974); J. E. Augustin et al., Phys. Rev. Lett. $\underline{33}, 1406$ (1974).

IV-6 G. Goldhaber et al., Phys. Rev. Lett. 37, 255 (1976); I. Peruzzi et al., Phys. Rev. Lett. 37,569 (1976); W. Braunscheig et al., Phys. Lett. $\underline{63 \mathrm{~B}}, 471$ (1976).

IV-7 B. Knapp et al., Phys. Rev. Lett. 37, 882 (1976).

IV-8 K. Niu et al., Prog. Theor. Phys. 46, 1644 (1971); N. Ushida et a1., Lett. Nuovo Cimento 23, 577 (1978); H. Fuch1 et al. Phys. Lett. $85 \mathrm{~B}, 135$ (1979).

IV-9 E. H. S. Burhop et al., Phys. Lett. 65B, 299 (1976); C. Angelini et al., Phys. Lett. 84B, 150 (1979).

IV-10 N. Ushida et al., Phys. Rev. Lett. 45, 1049 and 1053 (1980).

IV-11 R. Schindler thesis, SLAC Report No. 219 (1979); V. Luth, Proceedings of the 1979 International Symposium on Lepton and Photon Interactions at High Energies, Batavia (1979) p. 78; W. Bacino et al., Phys. Rev. Lett. 45, 329 (1980).

IV-12 S. P. Rosen, Phys. Rev. Lett. 44, 4 (1980); M. Bander, D. Silverman, A. Son1, Phys. Rev. Lett. 44,7 (1980); V. Barger, J. P. Leveille, P. M. Stevenson, Phys. Rev. D22, 693 (1980); H. Fritzsch, P. Minkowski, Phys. Lett. 90B, 455 (1980); K. Shizuya, LBL Preprint 11884, submitted to Phys. Lett.

IV-13 R. Ammar et al., Phys. Lett. 94B, 118 (1980).

IV-14 C. Angelini et al., Phys. Lett. $\underline{80 B}, 428$ (1979). 
IV-15 M. K. Gaillard, B. Lee, J. Rosner, Rev. Mod. Phys. 47, 277 (1975).

IV-16 R. Brandelik et al., Phys. Lett. 70B, 132 (1977); R. Brandelik et al., Phys. Lett. 80B, 412 (1979). 
CHAPTER V

CONCLUSIONS

Several conclusions can be drawn from the materlal presented in this dissertation. One is that this new experimental technique has proved its merit for detailed analysis of individual neutrino interactions. The NIKFI nuclear emulston which was used maintained good sensitivity at $30^{\circ} \mathrm{K}$ and was capable of withstanding the mechanical rigors of nearly one-half million expansions of the 15-Foot Bubble Chamber.

The combination of emulsion, bubble chamber and external muon identifier information permits the Identification of a high percentage of the produced particles. Because of the very large geometric acceptance, track parameters (e.g. momentum and angles) can be determined for essentially all particles created in the interactions. This capability is particularly important for the investigation of the lifetimes of charmed particles.

For example, at the time the details of the $F$ decay discussed in chapter III were published, these results gave the best determination of the mass of that particle: $2017 \pm 25 \mathrm{MeV} / \mathrm{c}^{2}$. Also the decay time, $1.4 \times 10^{-13}$ second, when combined with the two events from E531, permits a good determination of the lifetime of the $\mathrm{F}^{+}, 2.01(+1.80,-0.82) \times 10^{-13}$ seconds. The agreement between this value and the prediction of Shizuys's QCD calculation, $2.4 \mathrm{X}$ $10^{-13}$ second, offers support to the hypothesis that the Interactions of gluons cannot be neglected in examining the physics of the decays 
of charmed, pseudoscalar mesons. The lack of strange particles in the final state is also consistent with this hypothesis.

Several main results can be summarized about the production of hadrons in these events. The average number of produced hadrons is consistent with the Iinear increase with $\ln \left(W^{2}\right)$ observed in neutrino proton events. However, the dispersion of the multiplicity of these events rises more steeply with average multiplicity than in neutrino deuterium interactions. The observed slope is very close to that reported for $\mathrm{p}-\mathrm{p}$ collisions.

The ratio $\left.\left(D /<n_{c}\right\rangle\right)$ is independent of $N_{g}$ as predicted by the GLD model. The observed dependence on the atomic weight of the target, however, contradicts the GLD prediction that this number does not vary with $A$. Also the average number of grey tracks with respect to hadronic interactions is larger than the GLD predicts.

The normalized multiplicity results from this experiment are consistent with the CTM and the GLD models and are in disagreement with the MCM. Clear evidence for intranuclear cascading is seen both in the measured value of $R_{A}$ and in the rapidity distribution when compared with the distribution from neutrino - proton events. The ratio of the emulsion to proton rapidity distributions as a function of rapidity indicates somewhat more cascading (1.e. more A dependence) than predicted by the GLD model. The ratio of pseudorapidity distributions for the large $\mathrm{N}_{\mathrm{g}}$ and small $\mathrm{N}_{\mathrm{g}}$ samples is consistent with the prediction of the GLD model but also is consistent with being a flat distribution with value of unity. The data, when compared with pion - proton events at the same CTM 
effective $W$, show less $A$ dependence than that model pred1cts.

The transverse momentum distributions show no evidence for dependence upon $\mathrm{N}_{g}$ or upon $A$ at the rather low $\mathrm{p}_{t}^{2}$ values seen in the small sample of events examined. At this level of $p_{t}^{2}$ the transverse momentum seems to be inherent in the Initial $\mathrm{w}^{+}$- quark interaction and independent of the subsequent processes during the passage of the production products through the nucleus.

of the three models considered here, the Modified Cascade Model is the only one clearly inconsistent with the data. Neither the CTM nor the GLD model, however, is in complete agreement with these results. The Growth of Longltudinal Distances model gives a more detailed picture of the Interaction than the Coherent Tube Model and therefore generates a larger number of predictions accessible to empirical test. With some exceptions the GLD is in agreement with the results presented here but a larger data sample would provide an extremely interesting test of this model. In particular, neutrino nucleus events at high $Q^{2}$ should show a diffractive character if the GLD model is correct.

A second nuclear emulsion target was exposed to the FNAL neutrino beam by this collaboration recently. Experience gained from the first exposure and associated improvements in experimental technique should result in a significant increase in the size of this neutrino emulsion data sample. With this larger amount of data, new insights Into the nature of the decays of charmed particles and of neutrino nucleus interactions should be gained. 


\section{LIST OF REFERENCES}

Y. Afek et a1., Phys. Rev. D15, 2622 (1977).

Y. Afek et al., preprint TECHNION-PH-77-83 (1977).

R. Ammar et al., Phys. Lett. 94B, 118 (1980).

C. Angelini et al., Phys. Lett. $\underline{80 \mathrm{~B}}, 428$ (1979).

C. Angelini et al., Phys. Lett. $\underline{84 \mathrm{~B},} 150$ (1979).

J. J. Aubert et al., Phys. Rev. Lett. 33, 1404 (1974).

J. E. Augustin et a1., Phys. Rev. Lett. 33, 1406 (1974).

J. Babecki and G. Nowak, Acta Phys. Po1. B9, 401 (1978).

W. Bacino et al., Phys. Rev. Lett. $\underline{45}, 329$ (1980).

P. Bagnaia et al., University of Rome Internal Note No. 706 (1978).

M. Bander, D. Silverman, and A. Soni, Phys. Rev. Lett 44, 7 (1980).

V. Barger, J. P. Leveille, and P. M. Stevenson, Phys. Rev. D22, 693 (1980).

J. Bell et al., Phys. Rev. D19, 1 (1979).

A. Bialas and W. Czyz, Nucl. Phys. B137, 359 (1978).

G. Bialkowski, C. B. Chiu, and D. M. Tow, Phys. Rev. D17, 862 (1978).

J. D. Bjorken and J. Kogut, Phys. Rev. D8, 1341 (1973).

J. D. Bjorken, International Summer Institute on Theoretical Particle Physics, Hamburg (1975) p. 93.

J. Blietschau et al., Phys. Lett. 87B, 281 (1979).

C. S. Bogomolov et al., Proceedings of the Tenth International Conference on Solfd State Nuclear Track Detectors, Lyon (1979) P. 127.

R. Brandelik et al., Phys. Lett. 70B, 132 (1977).

R. Brandelik et al., Phys. Lett. $80 \mathrm{~B}, 412$ (1979). 
W. Braunscheig et al., Phys. Lett. 63B, 471 (1976).

E. H. S. Burhop et al., Phys. Lett. 65B, 299 (1976).

H. Burmeister and D. C. Cundy, CERN TC-L Internal Report No. 75-1 (1975).

T. H. Burnett, Proceedings of the 1977 International Symposium on Lepton and Photon Interactions at High Energies, Hamburg (1977) p. 227.

T. H. Burnett et al., Phys. Lett. 77B, 443 (1978).

J. W. Chapman et a1., Phys. Rev. D14, 5 (1976).

J. W. Chapman et al., Phys. Rev. Lett. $\underline{36}, 124$ (1976).

G. V. Davidenko and N. N. Nikolaev, Nuc1. Phys. B135, 333 (1978).

H. Deden et al., CERN EP-80-66 (1980).

J. E. Elias et al., Phys. Rev. D22, 13 (1980).

H. Fritzsch and P. Minkowski, Phys, Lett. 90B, 455 (1980).

H. Fuchi et al., Phys. Lett. 85B, 135 (1979).

M. K. Gaillard, B. Lee, and J. Rosner, Rev. Mod. Phys. 47, 277 (1975).

S. Glashow, J. Iliapoulous, and L. Maiani, Phys. Rev. D2, 1285 (1970).

G. Goldhaber et al., Phys. Rev. Lett. 37, 255 (1976).

L. Hand et al., Z. Phys. C1, 139 (1979).

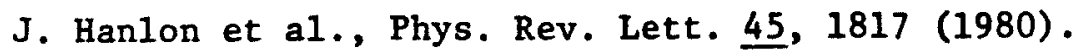

F. Hasert et al., Phys. Lett. $46 \mathrm{~B}, 121$ (1973).

F. Hasert et a1., Phys. Lett. 46B, 138 (1973).

H. G. Heilmann, CERN WA 21-1nt-1 (1978).

M. Hossain and D. Tow, Phys. Rev. D21, 1842 (1980).

T. Kitagaki et al., submitted paper No. 738 to the Twentieth

International Conference on High Energy Physics, Madison (1980).

B. Knapp et al., Phys. Rev. Lett. 37, 882 (1976). 
Z. Koba, H. B. Nielsen, and P. Olesen, Nucl. Phys. B40, 317 (1972). KODAK, KP 73555 1-77 (1977).

H. J. Lubatti et al., Z. Phys. C7, 241 (1981).

V. Luth, Proceedings of the 1979 International Symposium on Lepton and Photon Interactions at High Energies, Batavia (1979) p. 78.

J. H. Mulvey, Invited talk at the Summer Institute on Particle Physics; SLAC (1980).

M. J. Murtagh, Proceedings of the 1979 International Symposium on Lepton and Photon Interactions at High Energies, Batavia (1979) p. 277.

N. N. Nikolaev et al., CERN-TH-2541 (1978).

N. N. Nikolaev et al., CERN-TH-2575 (1978).

N. N. Nikolaev, Z. Phys. C5, 291 (1980).

K. Niu et al., Prog. Theor. Phys. 46, 1644 (1971).

K. Niu, Proceedings of the Twentleth International Conference on High Energy Physics, Madison (1980) p. 352.

I. Otterlund, University of Lund, Cosmic and Subatomic Physics Report LUIP 7711 (1977).

I. Perruzi et al., Phys. Rev. Lett 37, 569 (1976).

C. F. Powel1, P. H. Fowler, and D. H. Perkins, The Study of Elementary Particles by the Photographic Method, Pergamon Press, New York, 1959.

C. Quigg, FNAL PUB CONF 78/37 - THY (1978).

A. L. Read et al., Phys. Rev. D19, 1287 (1979).

S. P. Rosen, Phys. Rev. Lett. 44,4 (1980).

A. Salam and J. C. Ward, Phys. Lett. 13, 168 (1964).

R. Schindler, thesis SLAC Report No. 219 (1979).

N. Schmitz, Proceedings of the 1979 International Symposium on Lepton and Photon.Interactions at High Energies, Batavia (1979) P. 359.

K. Shizuya, LBL preprint 11884, submitted to Phys. Lett. 
W. Smart, FNAL TM-795 (1978).

N. Ushida et al., Lett. Nuovo Cimento $\underline{23}, 577$ (1978).

N. Ushida et al., Phys. Rev. Lett. 45,1049 (1980).

N. Ushida et al., Phys. Rev. Lett. 45, 1053 (1980).

I. Voyvodic et al., Fermilab proposal P564 (1977).

L. Voyvodic, Proceedings of the 1979 International Symposium on Lepton and Photon Interactions at High Energies, Batavia (1979) p. 569.

S. Weinberg, Phys. Rev. Lett. 19, 1264 (1967).

S. Weinberg, Phys. Rev. D5, 1412 (1972).

A. Wroblewski, Acta Phys. Pol. B4, 857 (1973).

W. M. Yeager et al., Phys. Rev. D16, 1294 (1977). 
VITA

Robert Ludwig Rosenbladt

High School: Stadium High School, Tacoma, Washington

Bachelor of Arts: Mathematics and Physics - Western Washington State College, Bellingham, Washington June 1974

Master of Science: University of Washington December 1976

Doctor of Philosophy: University of Washington June 1981 